\title{
Validating the Deployment of a Novel Tether Design for Net-Based Orbital Debris Removal Missions
}

\author{
by \\ Kevin Stadnyk \\ A thesis submitted to the Faculty of Graduate and Postdoctoral Affairs \\ in partial fulfillment of the requirements for the degree of
}

\author{
Master of Applied Science \\ in \\ Aerospace Engineering
}

\author{
Carleton University \\ Ottawa, Ontario
}

(C) 2020

Kevin Stadnyk 
This work is dedicated to my parents, Karen and Andrew Stadnyk, who's immense support for everything I do has allowed me to become the best person that I can be. 


\begin{abstract}
With the global push to commercialize space, humans are launching objects into orbit faster than natural effects are removing them. Orbital debris is especially dangerous as it is capable of exponential growth due to cascading collisions between orbiting objects. To ensure the long-term accessibility to space, high-risk objects must be actively removed to limit growth of the orbital debris population. One method of active debris removal is with a tethered-net to capture and tow an object out of orbit. This thesis continues the validation of a proposed novel tether configuration by focusing on its deployment dynamics. Tether elements are simulated using two numerical models, a lumped mass node system connected by massless spring-damper elements, and an absolute nodal coordinate formulation model. Their accuracy to predict the deployment motion of a tether is experimentally determined, and a complete capture scenario using the novel tether design is presented for the fist time.
\end{abstract}




\section{Acknowledgements}

I sincerely thank my supervisor, Steve Ulrich, for his guidance throughout my research and time at Carleton University and for the opportunity of this Masters of Applied Science degree.

I would also like to thank my parents, Karen and Andrew Stadnyk. Their support for my schooling, extracurriculars, and everything else I pursue cannot be overstated. They have sacrificed many things so that I could twice comfortably attend university while also focusing on my badminton career. For this, and everything else, I am eternally grateful. Their support has allowed me to become who I am today, and to continually strive to be the best that I can be. 


\section{Table of Contents}

Abstract $\quad$ iii

Acknowledgements $\quad$ iv

List of Tables viii

List of Figures $\quad$ ix

List of Symbols $\quad$ xi

List of Acronyms $\quad$ xiv

$\begin{array}{lll}\text { Chapter } 1 & \text { Introduction } & 1\end{array}$

1.1 Motivation ...................... 1

1.2 Problem Statement . . . . . . . . . . . . . . . . . . 3

1.3 Previous Work . . . . . . . . . . . . . . . . . . . . 4

1.4 Thesis Objectives . . . . . . . . . . . . . . . . . . 8

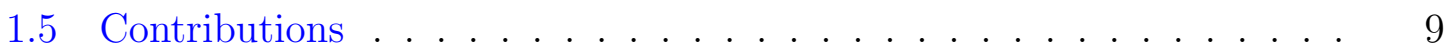

1.6 Organization ........................ 10

Chapter 2 On-Orbit Complete Capture Scenario 11

2.1 Introduction . . . . . . . . . . . . . . . . . . . . 11

2.2 On-Orbit Dynamics . . . . . . . . . . . . . . . . . . . . 11

2.2.1 Dynamics Formulation . . . . . . . . . . . . . 12

2.2.2 Attitude Motion . . . . . . . . . . . . . . . . . 13

2.2.3 Translational Motion . . . . . . . . . . . . . . . . . . 15

2.2.4 Deployment Dynamics Formulation - Lumped Mass SpringDamper Model . . . . . . . . . . . . . 16

2.2.5 Deployment Dynamics Formulation - Absolute Nodal Coordinate Formulation Model . . . . . . . . . . . . . . . . . 18 
2.2.6 Contact Dynamics Formulation - Impulse-Based Model . . . . 21

2.2.7 Post-Capture Dynamics Formulation - Spring-Damper Tether Model ..................... . . 26

2.2.8 Equations of Motion . . . . . . . . . . . . 26

2.3 On-Orbit Numerical Simulations . . . . . . . . . . . . . . . 28

$\begin{array}{lll}\text { Chapter } 3 & \text { Experimental Setup } & 37\end{array}$

3.1 Experimental Facility . . . . . . . . . . . . . . . . 37

3.2 Experimental Apparatus . . . . . . . . . . . . . . . . . 38

3.2.1 Tether Deployment Apparatus . . . . . . . . . . . . . . 39

3.2.2 Tether Bullet . . . . . . . . . . . . . . 40

3.2 .3 Target Impact Surface . . . . . . . . . . . . . . . . . . . . . . 42

3.2.4 Tether ...................... 43

Chapter 4 Planar Tether Deployment and Complete Capture Scenario 44

4.1 Introduction . . . . . . . . . . . . . . . . . . . 44

4.2 Planar Dynamics . . . . . . . . . . . . . . . . . 44

4.2.1 Dynamics Formulation . . . . . . . . . . . . . 46

4.2.2 Rotational Motion . . . . . . . . . . . . . . . 46

4.2.3 Translational Motion . . . . . . . . . . . . . . . . . . . 47

4.2.4 Planar Deployment Dynamics Formulation - Lumped Mass SpringDamper Model . . . . . . . . . . . . . . 48

4.2.5 Planar Deployment Dynamics Formulation - Absolute Nodal Coordinate Formulation . . . . . . . . . . . . 48

4.2.6 Planar Post-Capture Dynamics Formulation - Spring-Damper Tether Model . . . . . . . . . . . . . . . . . . . . . 49

4.2.7 Planar Equations of Motion . . . . . . . . . . . . 52

4.3 Planar Numerical Simulations . . . . . . . . . . . . . . . 54

4.4 Planar Tether Deployment Simulation . . . . . . . . . . . . 54

4.5 Planar Complete Capture Scenario Simulation . . . . . . . . . . 56 
$\begin{array}{lll}\text { Chapter } 5 & \text { Experimental Validation } & 64\end{array}$

5.1 Introduction . . . . . . . . . . . . . . . . . . . . . . . . . 64

5.2 Planar Tether Deployment Validation . . . . . . . . . . . . . 65

5.3 Complete Capture Scenario Results . . . . . . . . . . . . . . . . . 69

$\begin{array}{lll}\text { Chapter } 6 & \text { Conclusion } & 76\end{array}$

6.1 Thesis Summary . . . . . . . . . . . . . . . . . . . 76

6.2 Contributions to the Field of Space Debris Removal . . . . . . . . . 77

6.3 Practicality of this Work . . . . . . . . . . . . 78

6.4 Recommendations for Future Work . . . . . . . . . . . . . . . 79

$\begin{array}{lr}\text { Bibliography } & 80\end{array}$

$\begin{array}{lll}\text { Appendix A } & \text { Supplemental Figures } & 85\end{array}$

A.1 Experimental Tether Deployment Absolute Residual Plots . . . . . . 85 


\section{List of Tables}

2.1 Initial orbital elements of the on-orbit complete capture simulation. 29

2.2 Initial conditions and parameters of the tether deployment simulation. . . . . . . . . . . . . . . . . . 31

2.3 Initial conditions and parameters of the on-obrit post-capture simulation. ................... . . 31

4.1 Initial conditions and parameters for the planar tether deployment and post-capture scenario. . . . . . . . . . . . 55

$5.1 \quad$ Laboratory experiment parameters. . . . . . . . . 66

5.2 Average relative residuals of the spring-dapmer and ANCF models. 68 


\section{List of Figures}

$1.1 \quad$ Monthly number of objects in Earth orbit by object type. . . . 2

1.2 Single and sub-tether tethered-net configurations. . . . . . . 8

2.1 Reference frame and vector definitions for the dynamics modeling of both tether configurations. . . . . . . . . . . 13

2.2 Spring-Damper tether element and node pair. . . . . . . . 16

$2.3 \quad$ Single contact diagram of point $P$ with object $O$. . . . . . 22

$2.4 \quad$ Novel tether configuration deployment in an orbital environment using the massless spring-damper model. . . . . . . . . . 33

2.5 Novel tether configuration deployment in an orbital environment using the ANCF model. . . . . . . . . . . . . . . 34

2.6 Initial position of the TSS post-capture. . . . . . . . . 35

2.7 Detumbling motion of the tethered spacecraft system. . . . . 36

$3.1 \quad$ Overview of the Spacecraft Proximity Operations Testbed. . . 39

3.2 SPOT platforms. . . . . . . . . . . . . 40

3.3 Tether Deployment Payload. . . . . . . . . . . . . . 41

3.4 A tether bullet. . . . . . . . . . . . . . . . . 42

3.5 Target impact panel. . . . . . . . . . . . . . . . . . 42

4.1 Reference frame and vector definition for planar dynamics modeling. . . . . . . . . . . . . . . . . . 45

4.2 Novel tether configuration deployment in a planar environment using the massless spring-damper model. . . . . . . . . . . 58

4.3 Novel tether configuration deployment in a planar environment using the ANCF model. . . . . . . . . . . . . . . . . . 61

4.4 Platform positions and pointing angles during the simulated planar complete capture scenario. . . . . . . . . . . 63

$5.1 \quad$ Experimental scenario SPOT platform movement. . . . . . 65 
5.2 Spring-damper, ANCF, and experimental tether deployment of trial 2 at various time steps. . . . . . . . . . . . 72

5.3 Experiment 3 individual tether node absolute residuals over the duration of tether deployment. . . . . . . . . . . . 73

$5.4 \quad$ Spring-damper, ANCF, and experimental tether nodes initial positions. ...................... . . 74

5.5 Platform positions and pointing angles during the planar complete capture scenario. . . . . . . . . . . . . . 75

A.1 Trial 1 individual tether node absolute residuals over the duration of tether deployment. . . . . . . . . . . . . . 86

A.2 Trial 2 individual tether node absolute residuals over the duration of tether deployment. . . . . . . . . . . . 87

A.3 Trial 4 individual tether node absolute residuals over the duration of tether deployment. . . . . . . . . . . . . . 88

A.4 Trial 5 individual tether node absolute residuals over the duration of tether deployment. . . . . . . . . . . . . . 89

A.5 Trial 6 individual tether node absolute residuals over the duration of tether deployment. . . . . . . . . . . . . . 90

A.6 Trial 7 individual tether node absolute residuals over the duration of tether deployment. . . . . . . . . . . . . . . 91

A.7 Trial 8 individual tether node absolute residuals over the duration of tether deployment. . . . . . . . . . . . . . . . 92

A.8 Trial 9 individual tether node absolute residuals over the duration of tether deployment. . . . . . . . . . . . .

A.9 Trial 10 individual tether node absolute residuals over the duration of tether deployment. . . . . . . . . . . . . . 


\section{List of Symbols}

\begin{tabular}{ll}
$A$ & Cross-sectional area, $\left[\mathrm{m}^{2}\right]$ \\
$\mathbf{C}_{A B}$ & Rotation matrix from A to B \\
$E$ & Elastic Young's Modulus, $[\mathrm{Pa}]$ \\
$F$ & Force, $[\mathrm{N}]$ \\
$\mathbf{F}$ & Force vector components, $[\mathrm{N}]$ \\
$\overrightarrow{\mathcal{F}}_{x}$ & Vectrix of reference frame $x$ \\
$I$ & Area moment of inertia of a tether cross-section, $\left[\mathrm{m}^{4}\right]$ \\
$\mathbf{I}_{x \times x}$ & Identity matrix of dimension $x$ \\
$J$ & Moment of inertia, $\left[\mathrm{kg} \cdot \mathrm{m}^{2}\right]$ \\
$\mathbf{L}$ & Tether element stretch as vector components, $[\mathrm{m}]$ \\
$L_{0}$ & Tether element unstretched length, $[\mathrm{m}]$ \\
$\Delta L$ & Tether element stretch magnitude, $[\mathrm{m}]$ \\
$\mathbf{S}$ & Shape function \\
$S_{i}$ & Shape function component $i$ \\
$T$ & Kinetic energy, $\left[\mathrm{kg} \cdot \mathrm{m}^{2} / \mathrm{s}^{2}\right]$ \\
$U_{b}$ & Strain energy due to bending, $\left[\mathrm{kg} \cdot \mathrm{m}^{2} / \mathrm{s}^{2}\right]$ \\
$U_{l}$ & Strain energy due to longitudinal deformation, $\left[\mathrm{kg} \cdot \mathrm{m}^{2} / \mathrm{s}^{2}\right]$ \\
$\mathbf{a t t}_{x}$ & Tether attachment point on body $x$ with respect to center of mass, $[\mathrm{m}]$ \\
$c$ & Damping coefficient, $[\mathrm{N} \cdot \mathrm{s} / \mathrm{m}]$ \\
$\mathbf{d}$ & Deployment direction vector components of a tether bullet \\
$\mathbf{e}_{x}$ & Absolute nodal coordinates of a the tether segment at length $x$ \\
$k$ & Stiffness, $[\mathrm{N} / \mathrm{m}]$ \\
$m$ & Mass, $[\mathrm{kg}]$ \\
$\mathbf{q}$ & Quaternion \\
$\gamma$ & Position, $[\mathrm{m}]$ \\
& Newmark-beta integration coefficient \\
\hline & Newmark-beta integration coefficient \\
\hline &
\end{tabular}




\begin{tabular}{ll}
$\kappa$ & Curvature, $\left[\mathrm{m}^{-1}\right]$ \\
$\mu$ & Gravitational parameter of the Earth, $\left[\mathrm{m}^{3} / \mathrm{s}^{2}\right]$ \\
$\omega$ & Angular rate, $[\mathrm{deg} / \mathrm{s}]$ \\
$\phi$ & Tether angle, $[\mathrm{deg}]$ \\
$\rho$ & Fractional length along a tether element, $[\mathrm{m}]$ \\
$\tau$ & Density, $\left[\mathrm{kg} / \mathrm{m}^{3}\right]$ \\
$\theta$ & Torque, $[\mathrm{N} \cdot \mathrm{m}]$ \\
$\varepsilon_{l}$ & Angle, $[\mathrm{deg}]$ \\
$\zeta$ & Axial strain \\
\hline & Damping ratio
\end{tabular}

Subscript

$B$

$I$

$O$

$P$

b

$c$

control

end

$g$

$i$

impulse

$j$

$m$

$n$

$s$

$s_{i}$

$t$

thrust
Body-fixed reference frame

Inertial reference frame

The contact plane of object $O$

Mass point $P$

Refers to the bullet node of a tether

Refers to the chaser spacecraft

Refers to thrust used to actuate the SPOT platforms

Refers to the final node in a tether

Due to gravity

Refers to a specific number

Refers to an instantaneous impulse

Refers to the junction node

Refers to the main tether

Refers to a node of a tether

Refers to the single tether

Refers to sub-tether $i$

Refers to the target spacecraft

Refers to a continuous thrust 


$\begin{array}{ll}x & \text { Component in the } \overrightarrow{\mathbf{I}}_{x} \text { direction } \\ y & \text { Component in the } \overrightarrow{\mathbf{I}}_{y} \text { direction } \\ z & \text { Component in the } \overrightarrow{\mathbf{I}}_{z} \text { direction }\end{array}$

\section{Superscript}

T

$b$

$c$

$t$

$\times$

$-1$

$-$

$+$

\section{Conventions}

$x$

$\dot{x}$

$\ddot{x}$

$\vec{x}$

$\mathrm{X}$

$\|\mathbf{x}\|$ $\mathrm{X}$
Transpose

Body-fixed frame

Chaser body-fixed frame

Target body-fixed frame

Skew-symmetric matrix

Inverse of a matrix

Relative velocity of two objects before contact

Relative velocity of two objects after contact

An italic lowercase denotes a constant

A closed dot above denotes the time derivative

Two closed dots above denotes the second time derivative

An italic lowercase with an arrow denotes a vector

A bold lowercase denotes the three dimensional components of vector $\vec{x}$

Parallel vertical lines denotes the Euclidean norm of $\mathbf{x}$

A bold uppercase denotes a matrix 


\section{List of Acronyms}

$\begin{array}{ll}\text { ANCF } & \text { Absolute Nodal Coordinate Formulation } \\ \text { DOF } & \text { Degree of Freedom } \\ \text { ESA } & \text { European Space Agency } \\ \text { ESOC } & \text { European Space Operations Center } \\ \text { FEM } & \text { Finite Element Method } \\ \text { IADC } & \text { Inter-Agency Space Debris Coordination Committee } \\ \text { JAXA } & \text { Japan Aerospace Exploration Agency } \\ \text { JSS } & \text { Joined Spacecraft System } \\ \text { LEO } & \text { Low Earth Orbit } \\ \text { NASA } & \text { National Aeronautics and Space Administration } \\ \text { SPOT } & \text { Spacecraft Proximity Operations Testbed } \\ \text { SRCL } & \text { Spacecraft Robotics and Control Laboratory } \\ \text { TSS } & \text { Tethered Spacecraft System }\end{array}$




\section{Chapter 1}

\section{Introduction}

\subsection{Motivation}

The term orbital debris refers to objects in orbit around Earth that are not considered useful. Due to the vastness of space, orbital debris was thought to pose no risk to future spacefaring missions until proven otherwise by Donald J. Kessler in 1978 [1]. Known as the Kessler Syndrome, Kessler stated that eventual collisions between orbital objects will lead to sequential collisions, producing new debris, and ultimately create a chain reaction that will grow the debris population faster than it can naturally decay.

As of January 2019, the European Space Agency's (ESA) Space Debris Office at the European Space Operations Center (ESOC) estimated the total number of satellites which have been launched into orbit to be around 8950. Of these satellites, and estimated 5000 of them are still in orbit, while only 1950 of them are still functioning [2]. Large pieces of debris commonly occurs in the form of fragmented spacecraft pieces, spent launch vehicle stages, and nonoperational satellites. ESA's Space Debris Office reports that there are about 22300 of these objects tracked by global Space Surveillance Networks and maintained in their catalog [2]. Furthermore, there are many objects that are too small to be tracked. Over time, paint on satellites erodes away due to solar wind and friction from Earth's upper atmosphere. Collisions between these paint flecks, micrometeorites, and other small debris pieces is quietly increasing the total debris population.

Figure 1.1 offers a visual summary reported by the National Aeronautics and Space Administration (NASA) Orbital Debris Program Office of all orbital objects officially cataloged by the United States of America's Space Surveillance Network as of May 2019 [3]. Fragmentation Debris encompasses all satellite and anomalous event debris, while mission-related debris encompasses all objects intentionally separated 
from the spacecraft as part of a planned mission. It is important to note that only the fragmentation debris (pink), mission-related debris (orange), and rocket bodies (green) lines are considered orbital debris. Spacecraft still in orbit (blue) account for nearly one quarter of all tracked orbital objects. The amount of fragmented debris vastly outnumbers the amount of spacecraft, rocket bodies, and mission-related debris combined. This is largely due to two events in particular as seen by the two large spikes in the fragmentation debris plot. The first of these events occurred on January $11^{\text {th }} 2007$ when the Peoples Republic of China conducted a successful anti-satellite missile test using one of their unoperational weather satellites [4]. The second event occurred on February $10^{\text {th }} 2009$ between the operational US communications satellite Iridium 33 and Cosmos 2251 satellites [5]. Due to the extremely high relative velocities of orbital objects, orbital collisions can inject hundreds to thousands of new debris pieces with each occurrence.

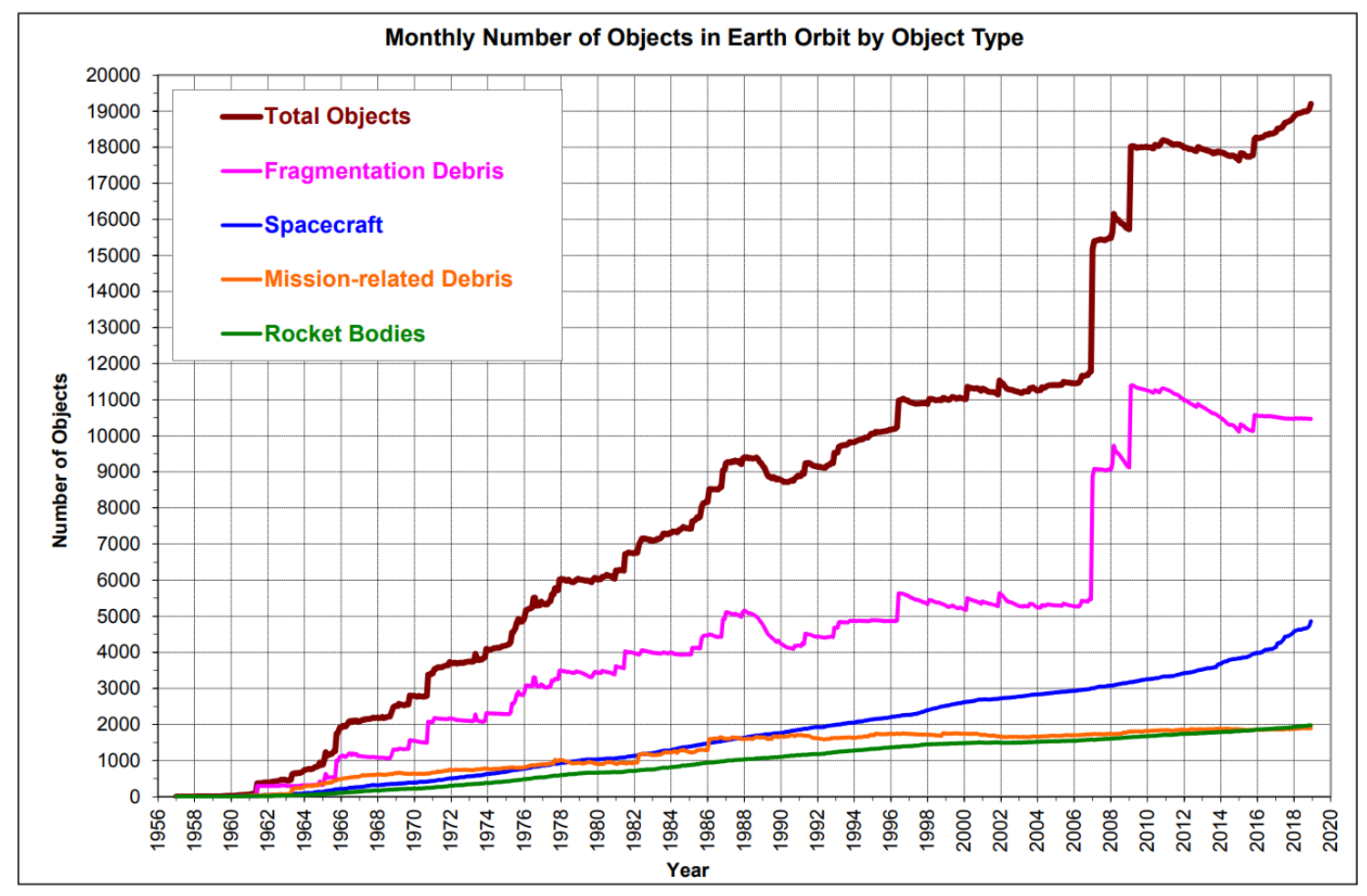

Figure 1.1: Monthly number of objects in Earth orbit by object type ${ }^{1}$.

\footnotetext{
${ }^{1}$ NASA Orbital Debris Program Office, Orbital Debris Quarterly News, Volume 23, Issue 1 \& 2, May 2019, pg. 13
} 
Beyond the number of objects that are capable of being tracked, and are publicly reported, statistical models predict that there are 34000 objects larger than $10 \mathrm{~cm}$ in diameter in orbit, an additional 900000 objects from ranging $1 \mathrm{~cm}$ to $10 \mathrm{~cm}$, and 128 million objects ranging from $1 \mathrm{~mm}$ to $1 \mathrm{~cm}[2]$.

To help combat the future increase of orbital debris, the Inter-Agency Space Debris Coordination Committee (IADC) enacted guidelines recommending that all spacefaring organizations ensure their orbital objects will naturally decay, or are maneuvered to an orbit in which the object will decay back to Earth, within 25 years of mission completion [6]. Currently, these are only guidelines and are not strictly enforced. The orbital debris population is still growing, and the solution to this problem lies in the active removal of select high-risk orbital debris. According to a NASA model, 5-10 high-risk debris objects must be removed from orbit each year to stabilize the low Earth orbit (LEO) environment [7]. Many different methods of active orbital debris removal have been proposed as a solution to this problem. These methods are broken down into three main categories that include: contactless capturing, rigid connection capturing, and flexible connection capturing.

One of the most promising methods of active debris removal lies within the flexible connection capturing category. The method in question uses a tethered-net to entrap the debris, before towing it to a lower altitude orbit. The configuration of tetherednets often takes the form of a single tether connecting the net to the spacecraft. Hovell and Ulrich [8-11] proposed and demonstrated a new tether configuration to improve the efficiency of the tethered-net active debris removal. The technology development of active debris removal, and the continued development and validation of this recently proposed tether configuration is the motivation behind this work.

\subsection{Problem Statement}

There are many phases of an active orbital debris removal mission with a tethered-net based payload. Of these, the deployment of the payload and subsequent stabilization of the tethered spacecraft system (TSS) are among the most important ones. In space, there is only one chance to capture a target when deploying a net and the captured object cannot be towed to a lower altitude if the post-capture TSS cannot 
be stabilized. To stabilize and eventually control a debris object that is tumbling with angular rates about all three axes, a novel tether design using the visco-elastic material properties of a flexible tether was proposed by Hovell and Ulrich [8-11]. This novel tether configuration is comprised of a single tether which branches into several sub-tethers with a net connected at their ends. Once the debris has been ensnared by the net, the visco-elastic properties of the sub-tethers use the motion of the tumbling target against itself, passively dampening its angular rates during the post-capture stabilization phase.

Experimental validation of the novel tether design and its angular rate dampening properties was preformed by Hovell and Ulrich [8-11]. It was concluded that if tension was maintained in the tether the passive dampening properties would have the greatest effect. However, all simulations and experiments used to reach this conclusion were preformed with both the chaser spacecraft and debris initially tethered together.

This thesis is concerned with the deployment phase of an active debris capture and removal mission using the novel tether design. It seeks to bridge the gap in the complete capture scenario by validating the deployment of the proposed novel tether design. It examines a scenario in which the the novel tether is deployed, contacts its target, and the resulting TSS is stabilized.

\subsection{Previous Work}

There has been a significant amount of research on the topic of space debris removal methods before the publication of this thesis. In this section, significant previously proposed methods of active orbital debris capture and removal are discussed in order to place the contributions of this work in the overall field of research. Following this, relevant research pertaining to flexible tethered nets is discussed.

All orbital debris methods can be broken down into three categories: contactless capturing, rigid connection capturing, and flexible connection capturing.

Contactless methods are desirable because any method in which the target and chaser are physically joined will influence the stability of the resulting joined spacecraft system (JSS). Complex orbit and attitude control is required to stabilize the 
JSS. Contactless methods are advantageous as they forgo this problem. One proposed method involves deorbiting the target using an artificial atmosphere [12]. This method looks to inject a cloud of atmospheric particles into the flight path of the debris, increasing the drag experienced, and subsequently degrading its altitude. An advantage of this method is that the atmospheric particles are considered to pose no threat to other orbital objects and will eventually fall back into the atmosphere. Disadvantages include the lack of control on the trajectory of the debris, and slow deorbiting times. Laser systems aim to shoot a pulse based laser beam which will positively charge the debris. It will then attract charged particles in LEO, increasing the drag experienced, and subsequently degrading its altitude [13]. An advantage of this method is that it is operational from the ground, greatly reducing both the associated risks and costs. Disadvantages include risk of the laser breaking the target and creating more debris, and that it is only effective on debris large enough to be tracked and within the field of view of the laser. Magnetic forces are being researched as a method of pseudo-grappling debris with high angular velocities about all three axes [14]. These spacecraft would interact with magnetorquers and other magnetic surfaces onboard spacecraft to pull them while maintaining a safe distance. Contactless methods are promising due to their capabilities of removing multiple targets from orbit during their lifetime. The main drawback of contactless methods are that they require an extremely long time to deorbit their targets, they are limited by their fuel capacity, and they are very complex. As a result this method is outpaced by the technological progress of rigid and flexible connection methods.

Rigid connections come in the form of one [15] or more [16] robotic arms or robotic tentacles [17] with a mechanical effector at the end of each limb. Robotic arms are currently the most understood technology with Canadarm 1 and 2 [18] being two successful examples of the technology. A disadvantage of this method is that the chaser is required to rendezvous at a close proximity with the target in order to capture it. Targets are commonly tumbling about all three axes, which requires a complex rendezvous procedure in order to match the angular rates. Additionally, in the case of one or more robotic arms the target must have a pre-installed and compatible grapple fixture for the end effector to latch to. To alleviate this problem, 
research has been done on end effectors with various grappling capabilities [19], and a clamp system with arms large enough for common targets can be used to grab the entirety of the target [20-22]. Once a secure connection is established, rigid connections are advantageous as the JSS acting as a single rigid body, allowing for easier control and stabilization of the system. Once stable, the chaser can tow or push the target to the desired orbit. Much like contactless methods, rigid connection methods are complex in the rendezvous procedures required, but are reusable and mainly limited by the available fuel for maneuvering.

Flexible connections are methods in the which a flexible tether is established between the target and chaser spacecraft. Electrodynamic tethers are a unique concept which were originally designed for maneuvering in LEO, but can be applied as a capture method [23]. Conceptually, a long electrically conductive cable stemming from the chaser spacecraft is attached to the target debris. The established tether attracts charged ions trapped in the upper atmosphere to become positively charged. It then interacts with the Earth's magnetic field to produce a deorbiting thrust [24]. Alternative applications see a spooled electrodynamic tether attached to the target, which then unspools to act as a gravity gradient boom, naturally stabilizing the debris, and passively deorbiting the target. The main disadvantages of this method include the complexity of the concept, and its reliance on Earth's magnetic field, limiting it to LEO applications only. This technology was first scheduled to be demonstrated as a payload aboard the Japan Aerospace Exploration Agency's (JAXA) Kounotori 6 International Space Station resupply vehicle. However, due to unknown complications the tether did not deploy and the technology demonstration was deemed a failure [25]. Harpoon capture consists of a barbed harpoon that is launched from the chaser into the target to establish the TSS [26,27]. For best performance, the harpoon aims to pierce the multi-layered insulation or empty fuel tanks of a target. Once the TSS has been established, it is stabilized before the target is towed to a lower altitude. Advantages of harpoon capture are that the debris can be captured from a distance reducing the risk of collisions, and the method is compatible with all types of sufficiently large debris. Disadvantages of this method includes the risk of the harpoon contacting wrong and not piercing the target, or if it strikes the target 
with too much force creating more debris in the process. This method was tested during the 2018 ESA RemoveDEBRIS demonstrator mission in which the deployed harpoon successfully penetrated into its target surface and established a tethered connection [28]. Net capture consists of corner masses of a net, denoted as bullets, which are attached to the net directly at the corners or by a short cable. These bullets are deployed at an expanding angle in the direction of the target to allow the net to expand from its packed form as it is pulled along. Upon contact with the target, the net will wrap around and become tangled within itself to secure the target. Alternative more complex but reliable methods such as maneuverable micro-spacecraft attached to the corners of the net [29] have also been proposed. The stabilization and deorbiting of the TSS is done using the same process as harpoon methods. Advantages to net capture are that the debris can be captured from a distance reducing the risk of collisions, and the capture method is compatible with all types of debris. Disadvantages include the risk of the tether becoming tangled, and the risk of the net wrapping around the target correctly in the case of net capturing. Net capture was also tested during the 2018 ESA RemoveDEBRIS demonstrator mission in which the deployed net successfully captured its target. However, no post-capture stabilization was preformed by the chaser spacecraft [28].

Of the aforementioned technologies, tethered-net capture is an early front runner. For this reason, ESA shortlisted the tethered-net method as one of two methods for their e.Deorbit mission [20]. This is because the technology required for netcapture is already well understood for terrestrial applications, and is quick to adapt for the orbital environment. The tethered-nets ability to capture a target of any shape and any size allows for it to be scaled in order to accommodate many different missions. Many net capture simulations [30-35] and experiments [28,36-39] have been preformed to model how a net will interact with a target in an orbital environment. Specifically, dynamic models of a net using a lumped-mass model [40,41], an absolute nodal coordinate formulation (ANCF) model [41,42], an elastic continuum model [43], and a cubic B-spline mode [44] have been previously discussed.

However, this work omits modeling a net attached to the tether due to the limitations of the laboratory facility used. The laboratory is only capable of preforming 
experiments in a planar environment. This was determined to be unsuitable for a net as it requires six degreed of freedom to properly wrap around a target. As a result, this work specifically focuses on the tether portion of the tethered-net configuration. It presents planar tethers in both experimentally and in simulation, and further presents the tethers in an orbital capture scenario. Models of tethered spacecraft were originally proposed by Carter and Green [45] as a series of beads connected by springs and dashpots. Hovell [46] details a comprehensive list of previous work on the topic of tether usage for net and harpoon capture. In his work Detumbling Space Debris Using Tethers [46], Hovell expands on the topic of traditional single tether configurations, as seen in Fig. 1.2(a), by presenting a novel sub-tether configuration, as seen in Fig. 1.2(b), and the utilization of intrinsic nonlinear visco-elastic properties during the post-capture stabilization phase to stabilize a uncooperative tumbling target. Hovell's [46] work which concentrated on the stabilization phase determined that a sub-tether configuration with multiple tethers branching out to the target spacecraft from the main tether would be capable of reducing the angular rate of the target faster than a single tether connecting the chaser and target.

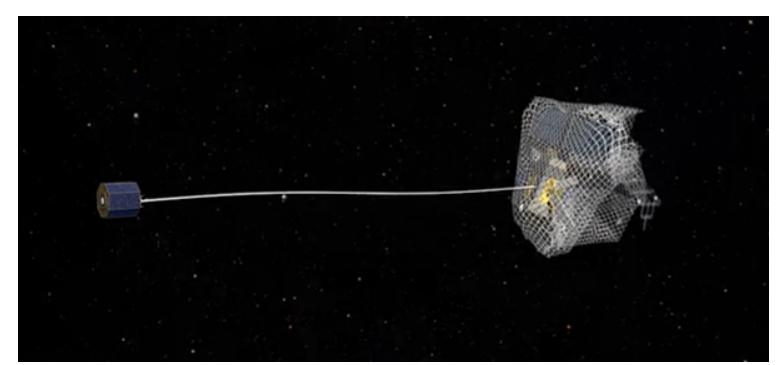

(a) Single tether configuration

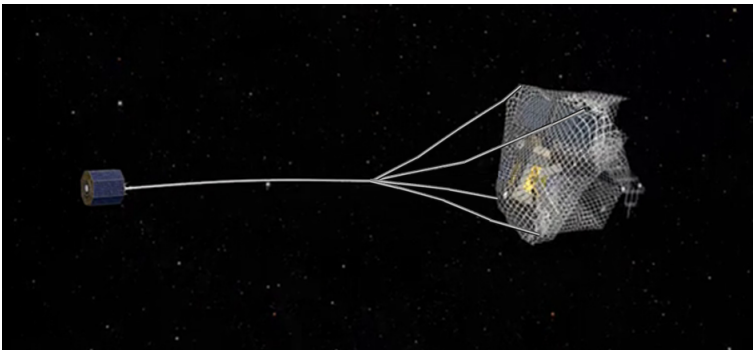

(b) Sub-tether configuration

Figure 1.2: Single and sub-tether tethered-net configurations ${ }^{2}$.

\subsection{Thesis Objectives}

This work examines the deployment phase of an active space debris capture mission using the proposed novel tether design. It looks to build on work done by Hovell [46]

\footnotetext{
${ }^{2}$ ESA, Space debris 2013, 2013 (accessed July, 2019) http://www.esa.int/spaceinvideos/ Videos/2013/04/Space_debris_story
} 
and Hovell and Ulrich [8-11] by combining the deployment and stabilization phases in order to demonstrate, for the first time, a complete capture scenario using the proposed novel tether design. Analyzing the deployment motion of the novel tether design is important as it allows for the confirmation that the sub-tethers will not tangle during flight and that the novel configuration will deploy at the intended speed and in the intended direction. In addition, a method for modeling the contact dynamics between the target debris and tethers is presented as a basis for future improvements to be done on this work.

To accomplish this, the deployment of the novel tether is simulated using two different numerical models: a massless spring-damper element with lumped mass nodes model, and an ANCF model. To simulate the contact phase an impulse-based model is used. These models are used to simulate the tether deployment in both a orbital environment and a planar environment replicating the conditions used to experimentally validate the numerical models.

In an experimental planar environment, the deployment and post-capture stabilization phases are validated between a chaser and target with differing angular rates, thereby emulating a non-ideal pose tracking maneuver. The planar numerical simulations are compared to the planar experimental results to determine how accurate the two presented numerical models are in predicting the deployment motion of the novel tether configuration. This comparison will determine which of the discussed models are appropriate to use when simulating a complete active debris capture scenario.

\subsection{Contributions}

The contributions of this work to the field of tethered space debris removal are:

- Determining the effectiveness of the spring-damper and ANCF numerical models used in predicting the motion of the sub-tether configuration.

- Implementing impulse-based contact dynamics for a harpoon scenario using the sub-tether configuration.

- Demonstrating the sub-tether configuration in a complete capture scenario in both simulations and experiments. 
- The development of a tether deployment payload for Carleton University's Spacecraft Proximity Operations Testbed (SPOT) platforms.

\subsection{Organization}

This thesis is organized as follows:

Chapter 2: On-Orbit Complete Capture Scenario presents a complete capture scenario using the novel tether configuration in a simulated LEO environment. The spacecraft equations of motion, two different deployment phase tether models, a contact dynamics model, and a post-capture stabilization tether model are presented along with a numerical simulation and results of the complete capture scenario.

The tether deployment apparatus constructed for Carleton University's SPOT is presented in Chap. 3: Experimental Setup. This chapter provides a high-level overview of the SPOT and its validation capabilities within the fields of space dynamics, guidance, navigation, and control theories. It details the author's contribution to the SRCL in the form of the tether deployment apparatus created for the SPOT.

Planar simulation models for the deployment and stabilization phases of the planar tether model validation and complete capture scenario are presented in Chap. 4: Planar Tether Deployment and Complete Capture Scenario, which simulates an environment of the SPOT. The planar equations of motion, differences in the two tether deployment models and post-capture stabilization models are presented along with numerical simulations of the tether deployment using the two tether models and the planar complete capture scenario.

Chapter 5: Experimental Validation discusses the experimentally reproduced results of the planar tether deployment and full capture scenario experiments, and comments on the effectiveness of the presented numerical tether deployment models.

Lastly, Chap. 6: Conclusion summarizes the work presented in this thesis, comments on the practicality of the proposed tether configuration during the deployment phase of a debris removal mission, and the validity of the presented numerical models in replicating the deployment of the novel tether configuration. 


\section{Chapter 2}

\section{On-Orbit Complete Capture Scenario}

This chapter presents the methods used for the dynamical modeling of chaser and target spacecraft and tethers in low Earth orbit (LEO). This model includes perturbations encountered in the LEO environment, specifically orbital motion and gravitygradient torques, as well as forces due to tether deployment and contact between the tether and target spacecraft.

\subsection{Introduction}

High fidelity numerical simulations are useful for understanding how a tether is deployed from a spacecraft in an orbital environment. By modeling the dynamics of a chaser spacecraft and tether, including perturbations present in the orbital environment, an accurate tether deployment scenario can be investigated. This chapter presents the dynamical motion of a chaser and target spacecraft, two tether models, and the post-capture tethered spacecraft system (TSS) dynamics. It builds on work from Hovell [46] to bring together the deployment and post-capture stabilization phases of an active debris removal scenario in order to demonstrate a complete capture scenario using the novel tether.

\section{$2.2 \quad$ On-Orbit Dynamics}

The tether deployment scenario consists of two spacecraft. First is an actively controlled spacecraft (the chaser), while the second is an uncooperative spacecraft (the target) that is tumbling about all three axes. The chaser and tether nodes are modeled as a point masses while the target is modeled as a rectangular prism. Similarly to Shan [41] who studied space-based net dynamics, the tethers deployment dynamics in this thesis are modeled with two different numerical models. The first model 
presented is comprised of lumped mass nodes connected by massless spring-damper elements. The second model approximates the tethers using absolute nodal coordinate formulation $(\mathrm{ANCF})$ beam elements that are so thin they act as flexible tethers. In the post-capture stabilization phase the tether is comprised of massless springdamper elements that connect at a single node. All tether models ignore torsion. Additionally, the spring-damper models ignores bending effects. A net modeled at the end of the tethers is omitted as it is not compatible in the laboratory environment used to validate the numerical models.

Initially, the tether is attached to the chaser spacecraft and folded within a single point. Upon deployment the tether bullets are each deployed along an unique angle called the deployment angle, $\theta_{d_{i}}$, with $i$ representing a given sub-tether. This causes the sub-tethers to radiate outward before contacting the target. Impulse forces of the tether deployment and the contact between the sub-tethers and target are accounted for. Figure 2.1 shows the configuration of the tethered spacecraft system (TSS) in the post-capture stabilization phase. The tether leaving the chaser is denoted as the main tether and the four tethers connecting the main tether to the target are denoted as the sub-tethers. The point where the main tether splits into sub-tethers is denoted the junction.

This section presents the equations used to simulate the chaser and target spacecraft, the tether during deployment and post-capture, and the contact between the tether and target.

\subsubsection{Dynamics Formulation}

In constructing the orbital environment of the spacecraft and tethers, the reference frame $\mathcal{F}_{I}$ represents an inertially-fixed reference frame, and the position of the target is described by the position vector $\vec{r}_{t}$. These are used to calculate $\mathbf{r}_{t}$, representing the three-dimensional components of the target's center of mass position vector in the inertially fixed reference frame

$$
\vec{r}_{t}=\overrightarrow{\mathcal{F}}_{I}^{\mathrm{T}} \mathbf{r}_{t}
$$

The chaser and all tether nodes are represented as point masses. Their locations 


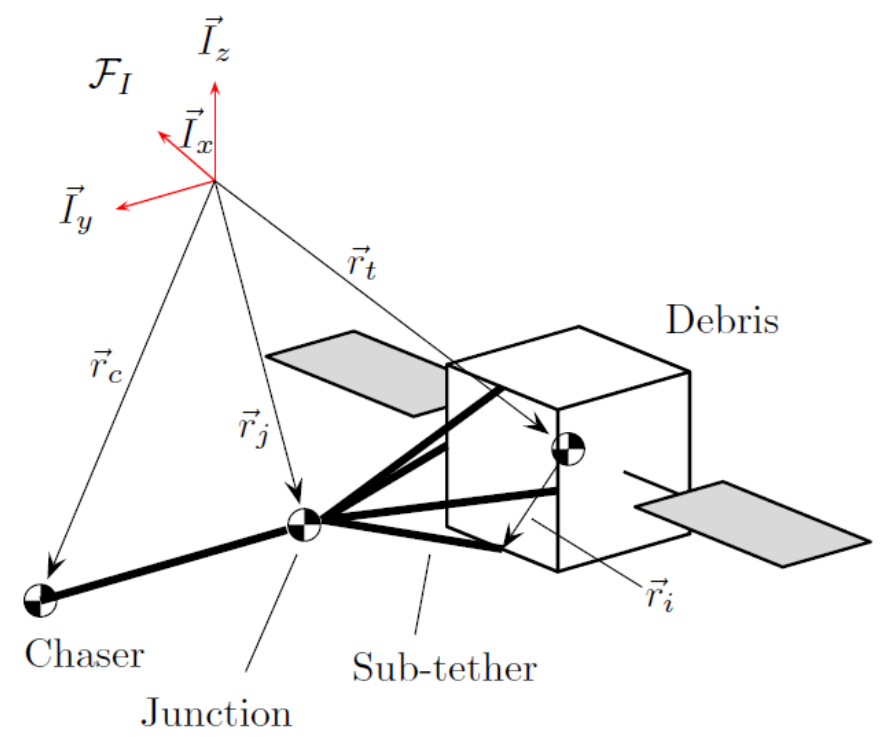

Figure 2.1: Reference frame and vector definitions for the dynamics modeling of both tether configurations [46].

are respectively described by position vectors $\vec{r}_{c}$ and $\vec{r}_{n_{i}}$, respectively, such that:

$$
\begin{aligned}
\vec{r}_{c} & =\overrightarrow{\mathcal{F}}_{I}^{\mathrm{T}} \mathbf{r}_{c} \\
\vec{r}_{n_{i}} & =\overrightarrow{\mathcal{F}}_{I}^{\mathrm{T}} \mathbf{r}_{n_{i}}
\end{aligned}
$$

where $\mathbf{r}_{c}$ and $\mathbf{r}_{n_{i}}$ are the three-dimensional components of the chaser and $i^{\text {th }}$ node position vectors in the inertially fixed reference frame, respectively.

\subsubsection{Attitude Motion}

Euler's equations of motion are used to describe the target spacecraft angular rates in the body-fixed reference frame $\mathcal{F}_{B}$. This is given by: [47]

$$
\mathbf{J} \dot{\omega}+\omega^{\times}(\mathbf{J} \boldsymbol{\omega})=\tau
$$

where $\boldsymbol{\omega}$ is the components of the angular rate vector in $\mathcal{F}_{B}, \mathbf{J}$ is the inertia matrix of the target, and $\boldsymbol{\tau}$ is the sum of external torque applied to the target.

By assuming that $\mathcal{F}_{B}$ is aligned with the principal axes of the body, $\mathbf{J}$ can be simplified to: 


$$
\mathbf{J}=\left[\begin{array}{ccc}
J_{x x} & 0 & 0 \\
0 & J_{y y} & 0 \\
0 & 0 & J_{z z}
\end{array}\right]
$$

where $J_{x x}, J_{y y}$, and $J_{z z}$ are the principal moments of inertia of the target spacecraft.

The attitude of the target spacecraft is described using quaternion kinematics: [47]

$$
\dot{\mathbf{q}}=\frac{1}{2}\left[\begin{array}{c}
\left(\boldsymbol{\epsilon}^{\times}+\eta \mathbf{I}_{3 \times 3}\right) \boldsymbol{\omega} \\
-\boldsymbol{\epsilon}^{\mathrm{T}} \boldsymbol{\omega}
\end{array}\right]
$$

where $\mathbf{q}$ represents the attitude quaternion in $\mathcal{F}_{B}$ with respect to the inertially-fixed reference frame, $\mathcal{F}_{I}$. It is defined as:

$$
\mathbf{q}=\left[\begin{array}{l}
q_{1} \\
q_{2} \\
q_{3} \\
q_{4}
\end{array}\right]=\left[\begin{array}{l}
\boldsymbol{\epsilon} \\
\eta
\end{array}\right]=\left[\begin{array}{c}
\mathbf{b} \cos \left(\frac{\phi}{2}\right) \\
\sin \left(\frac{\phi}{2}\right)
\end{array}\right]
$$

where $\mathbf{b}$ is the axis of rotation, $\phi$ is the angle of rotation, and

$$
\begin{gathered}
\boldsymbol{\epsilon}=\left[\begin{array}{l}
q_{1} \\
q_{2} \\
q_{3}
\end{array}\right] \\
\eta=q_{4} .
\end{gathered}
$$

An additional perturbation affecting the spacecraft attitude is the gravity gradient torque, which is caused due to the inverse square law of the gravitational field of the Earth. This causes an unequal gravitational force acting across the spacecraft body, leading to restoring torques acting on the spacecraft [47]. These restoring torque effects are significant in LEO. This effect is quantified through:

$$
\boldsymbol{\tau}_{g}=\frac{3 \mu}{\left\|\mathbf{r}_{t}^{b}\right\|^{5}} \mathbf{r}_{t}^{b \times} \mathbf{J r}_{t}^{b}
$$


where $\boldsymbol{\tau}_{g}$ is the resulting gravity-gradient torque components in $\mathcal{F}_{B}, \mu$ is the gravitational parameter of Earth, and $\mathbf{r}_{t}^{b}$ are the components of the target position vector expressed in $\mathcal{F}_{B}$, as:

$$
\mathbf{r}_{t}^{b}=\mathbf{C}_{\mathrm{BI}}(\mathbf{q}) \mathbf{r}_{t} .
$$

where the rotation matrix $\mathrm{C}_{\mathrm{BI}}(\mathbf{q})$ represents a rotation from $\mathcal{F}_{I}$ to $\mathcal{F}_{B}$. This is obtained from the quaternion $\mathbf{q}$ by:

$$
\mathbf{C}_{\mathrm{BI}}(\mathbf{q})=\left[\begin{array}{ccc}
1-2 q_{2}^{2}-2 q_{3}^{2} & 2\left(q_{1} q_{2}+q_{4} q_{3}\right) & 2\left(q_{1} q_{3}-q_{4} q_{2}\right) \\
2\left(q_{1} q_{2}-q_{4} q_{3}\right) & 1-2 q_{1}^{2}-2 q_{3}^{2} & 2\left(q_{2} q_{3}+q_{4} q_{1}\right) \\
2\left(q_{1} q_{3}+q_{4} q_{2}\right) & 2\left(q_{2} q_{3}-q_{4} q_{1}\right) & 1-2 q_{1}^{2}-2 q_{2}^{2}
\end{array}\right]
$$

\subsubsection{Translational Motion}

To model the target, chaser, and tether nodes linear motion, Newton's second law is used:

$$
\mathbf{F}=m \ddot{\mathbf{r}}
$$

where $\mathbf{F}$ is the net force applied to the body, $m$ is the mass of the body in question, and $\ddot{\mathbf{r}}$ is the inertial linear acceleration.

The force due to gravity acting on an orbiting spacecraft or tether node, $\mathbf{F}_{g}$, is described by Newton's law of gravitation

$$
\mathbf{F}_{g}=-\frac{\mu m}{\|\mathbf{r}\|^{3}} \mathbf{r}
$$

where $\mathbf{r}$ is the components of the position vector of the orbiting spacecraft or tether node in $\mathcal{F}_{I}$. Newton's law of gravitation applies to the chaser, target, and tether nodes.

During deployment while the tether is not under tension, forces exerted on the chaser spacecraft are due to the impulse generated during tether deployment and it's orbital motion. No forces due to the tether are exerted on the target spacecraft. During the contact phase, forces are exerted on the target as a result of the tether 
impact. Post-contact, the main tether and sub-tethers generate external forces while in tension which are applied to each mass.

\subsubsection{Deployment Dynamics Formulation - Lumped Mass Spring-Damper Model}

As shown in Fig. 2.2, the lumped mass node and massless spring-damper model approximates the tether as a series of lumped mass points, called nodes, connected by massless spring-damper elements. The tether can be discretized into any number of spring-damper and node pair sections. Although more nodes will increase the resolution of the movement of the tether, it was observed that this process has diminishing returns if too many are added [39].

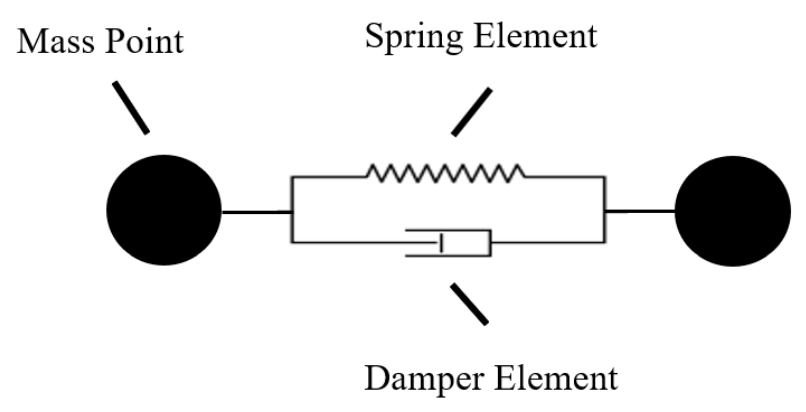

Figure 2.2: Spring-Damper tether element and node pair.

In defining the tether characteristics, a tension force is only generated in a tether element when it is elongated greater than its initial resting length, and elements are not capable of withstanding compression. In this application, the tether is characterized as a linear Kelvin-Voigt material. It is defined as a visco-elastic material having the properties both of elasticity and viscosity and is represented by a purely elastic spring and a purely viscous damper connected in parallel. This model is used to efficiently characterize the tension force in the cable. Based on this model, the tension force, $\mathbf{F}_{i k}$, between nodes $i$ and $k$ is conditioned as:

$$
\mathbf{F}_{i k}= \begin{cases}F_{i k} \frac{\mathbf{r}_{i k}}{\left\|\mathbf{r}_{i k}\right\|}, & \text { for } r_{i k}>L_{0} \\ 0, & \text { otherwise }\end{cases}
$$


where $\mathbf{r}_{i k}$ is the position vector along the $i^{\text {th }}$ and $k^{\text {th }}$ nodes in $\mathcal{F}_{I}, L_{0}$ is the initial unstretched length of the tether element. The force acting on the $i^{\text {th }}$ node by the $k^{\text {th }}$ node, $F_{i k}$, is expressed as:

$$
F_{i k}=-k\left(r_{i k}-L_{0}\right)-c \dot{r}_{i k}
$$

where $k$ is the axial stiffness of the tether, $c$ is the damping coefficient of the tether, and $r_{i k}$ and $\dot{r}_{i k}$ are the relative positions and velocities between the $i^{\text {th }}$ and $k^{\text {th }}$ nodes in $\mathcal{F}_{I}$.

By assuming the tether material is a homogeneous isotropic linear material, the axial stiffness of the tether, $k$, is defined as:

$$
k=\frac{E A}{L_{0}}
$$

where $E$ is the elastic Young's modulus of the tether material, and $A$ is the crosssectional area of the tether.

The damping coefficient is dependent on the material. It is a function of the node mass, $m_{i}$, stiffness, and damping ratio, $\zeta$, given as:

$$
c=2 \zeta \sqrt{m_{i} k}
$$

The lumped mass distribution of each node is modeled as the half-masses of the number of adjacent nodes and the cross-sectional area of each tether segment to any given node. The tether has three distinct node masses, with the mass of the $i^{\text {th }}$ node given as:

$$
m_{i}= \begin{cases}m_{b}, & \text { for } i \text { at the tether end } \\ \rho A L_{0}, & \text { for } i \text { along the tether } \\ 3 / 2 \rho A L_{0}, & \text { for } i \text { at the tether junction }\end{cases}
$$

where $m_{b}$ is the mass of the tether bullet and $\rho$ is the material density of the tether.

Using Eqs. (4.10) and (2.19), the dynamic equations of motion for the entire tether in $\mathcal{F}_{I}$ is discretized as: 


$$
m_{i} \frac{d \dot{\mathbf{r}}_{i}}{d t}=\sum_{k=1}^{N_{i}} \mathbf{F}_{i k}+\mathbf{F}_{g}
$$

where $N_{i}$ is the number of adjacent nodes to node $i$.

\subsubsection{Deployment Dynamics Formulation - Absolute Nodal Coordinate Formulation Model}

In traditional applications of finite element methods (FEM), assumptions about the small deformations and rotations of an element are made, which results in those modeling methods not accurately describing the dynamic characteristics of a highly flexible system, such as the novel tether in question. To circumvent this problem, absolute nodal coordinate formulation is used. ANCF was first proposed by Shabana et al. [48] and is most commonly found in applications related to beam bending or large displacement and deformation problems [49]. The ANCF method stems from FEM, but differs in that the absolute positions and the gradients of those nodal positions act as the element nodal coordinates to describe the configuration of a flexible system [48], whereas in traditional FEM infinitesimal small, or finite rotations, are used to describe the orientation of the elements.

This differentiation allows for the mass matrix of an element to remain constant when deriving the ANCF dynamic equations of motion. Additionally, since the nodal coordinates of the elements are defined in a global inertial coordinate frame there is no need for transformations to be applied on the elements. This is contrasted by the complex elastic force models, which are non-linear and coordinate or time dependent. Simplified models of these elastic forces have been derived by Berzeri and Shabana [50]. In simplifying the tether further, Gerstmayr and Shabana [51] derived a low-order tether element that has half of the degrees of freedom of a fully parameterized beam element, resulting in the tether element only accounting for longitudinal deformation. In this section, the dynamic equations of motion for the rigid-flexible coupled system for the novel tether are derived.

In using an ANCF model, a tether is approximated by a beam with a very small cross-sectional area relative to its length, allowing it to become very flexible. Each 
tether element contains a node each end. The absolute nodal coordinates, $\mathbf{e}_{i}$, of a given node in tether element are comprised by the global displacement and slope of the element, $\mathbf{r}_{i}$ and $\mathbf{r}_{i x}$, respectively. It is expressed as:

$$
\mathbf{e}_{i}=\left[\mathbf{r}_{i}, \mathbf{r}_{i x}\right]
$$

where

$$
\mathbf{r}_{i x}=\frac{\partial \mathbf{r}_{i}}{\partial x}
$$

An arbitrary position in a cable element, $\mathbf{r}_{x}$, is determined by the shape function, $\mathbf{S}$, and the absolute nodal coordinates of the tether at the lengths of $x=0$ and $x=L_{0}$. This is expressed as:

$$
\mathbf{r}_{x}=\mathbf{S e}=\left[\mathbf{S}_{1} \mathbf{I}_{3 \times 3}, \mathbf{S}_{2} \mathbf{I}_{3 \times 3}, \mathbf{S}_{3} \mathbf{I}_{3 \times 3}, \mathbf{S}_{4} \mathbf{I}_{3 \times 3}\right]\left[\mathbf{e}_{x=0}, \mathbf{e}_{x=L_{0}}\right]^{T}
$$

with the elements of the shape function taking the form:

$$
\begin{aligned}
& S_{1}=1-3 \psi^{2}+2 \psi^{3} \\
& S_{2}=L_{0}\left(\psi-2 \psi^{2}+\psi^{3}\right) \\
& S_{3}=3 \psi^{2}+2 \psi^{3} \\
& S_{4}=L_{0}\left(-v^{2}+\psi^{3}\right)
\end{aligned}
$$

where $\mathbf{I}_{3 \times 3}$ is the $3 \times 3$ identity matrix, $\psi$ is the fractional length along the tether where $\psi=\frac{x}{L_{0}}$, and $x$ is the coordinate of an arbitrary point along the tether element. It is assumed that the $y$ and $z$ directions are not accounted for because the magnitude of the displacement in those directions is very small compared to the magnitude of the displacement in $x$.

The constant mass matrix of a tether element, $\mathbf{M}_{i}$ is derived by evaluating the kinetic energy expression of a tether element, $T_{i}$. This is expressed as:

$$
T_{i}=\frac{1}{2} \int_{V} \rho \dot{\mathbf{r}}_{i}^{T} \dot{\mathbf{r}}_{i} d V=\frac{1}{2} \dot{\mathbf{e}}_{i}^{T} \mathbf{M}_{i} \dot{\mathbf{e}}_{i}
$$

where $\rho$ is the material density of the tether element and $\dot{\mathbf{r}}_{i}$ is the velocity components 
of the tether element in $\mathcal{F}_{I}$. The constant mass matrix can be simplified to:

$$
\mathbf{M}_{i}=\int_{V} \rho \mathbf{S}^{T} \mathbf{S} d V
$$

When the tether is in tension, elastic forces are applied based on an elastic energy model. The elastic forces are made up of two parts: the strain energy due to longitudinal deformation, $U_{l}$, and the strain energy due to bending, $U_{b}$. They are expressed as:

$$
\begin{aligned}
U_{l} & =\frac{1}{2} \int_{0}^{L_{0}} E A \varepsilon_{l}^{2} d x \\
U_{b} & =\frac{1}{2} \int_{0}^{L_{0}} E I \kappa^{2} d x
\end{aligned}
$$

where the axial strain, $\varepsilon_{l}$, is expressed based on CauchyGreen longitudinal strain, and the curvature, $\kappa$, is derived from the SeretFrenet frame [51]. The area moment of inertia of tether cross-section is denoted by $I$.

Using the strain energy, the elastic forces are calculated as:

$$
\mathbf{F}_{i}=\left(\frac{\partial U}{\partial \mathbf{e}_{i}}\right)^{T}
$$

Acceleration, velocity, and position of the tether nodes are calculated using the Newmark-beta integration method. It uses the extended mean value theorem, and states that the first time and second time derivatives can be solved as:

$$
\dot{u}_{n+1}=\dot{u}_{n}+\Delta t\left\{(1-\gamma) \ddot{u}_{n}+\gamma \ddot{u}_{n+1}\right\}
$$

and

$$
u_{n+1}=u_{n}+\Delta t \dot{u}_{n}+\frac{1}{2} \Delta t^{2}(1-2 \beta) \ddot{u}_{n}+2 \beta \ddot{u}_{n+1}
$$

where $\gamma$ and $\beta$ are coefficients bounded by $0 \leq \gamma \leq 1$ and $0 \leq 2 \beta \leq 1$ respectively. These coefficients $\gamma$ and $\beta$ can be tuned to output various results. 


\subsubsection{Contact Dynamics Formulation - Impulse-Based Model}

As this work only looks at the deployment of the tethers and omits a net attached at the end, only the bullet nodes of the tether contact the surface of the target. This contact is considered instantaneous and is applied to both objects at the beginning of the post-capture detumbling phase when the model used to simulate the tethers is changed. In modeling how the tethers behave on contact, an impulse-based method [52] is used. The impulse-based method models the impulse between two contacting objects, in this case the tether bullet and target surface, and sets new velocities for both of these objects. The impulse is caused by the contact instead of a calculated contact force. The immediate velocity changes of the two objects prevents interpenetration, as the bodies begin to move away from each other once contact occurs. The impulse-based method is commonly used in describing the contact between two separate bodies [53] and has been extensively implemented in simulating contact within virtual environments $[54,55]$.

Mirtich et al. [53] consider that the impulse-based method has a weakness on the handling of multiple simultaneous contacts. Shan [41] applied and verified multiple and simultaneous contacts using the impulse-based contact dynamic model for the first time in his modeling of a net capturing a space debris object. The same methods are applied for the multiple and simultaneous contacts of the sub-tethers in the novel tether configuration. This section outlines both the single and multiple contacts of a target and tether bullet.

In the single contact model a single mass point $P$ contacts a tumbling object $O$ with an angular velocity $\omega_{O}$ as shown in Fig. 2.3. The three element position vector of the mass point and the center of mass of the object are $\mathbf{r}_{P}$ and $\mathbf{r}_{O}$ respectively. Similarly, the velocities are given by $\mathbf{v}_{P}$ and $\mathbf{v}_{O}$, and lastly, $\mathbf{n}$ represent the normal direction of the contact plane on object $\mathrm{O}$.

To determine if the tether bullets are contacting the target, contact detection is preformed at every step of the simulation. Contact detection checks if the position of a given tether bullet, $\mathbf{r}_{P_{i}}$, is within an allowable area threshold of the target spacecraft. If the relative distance becomes too small, then the contact dynamics are implemented on the two objects. This method of contact detection is possible as there are only four 

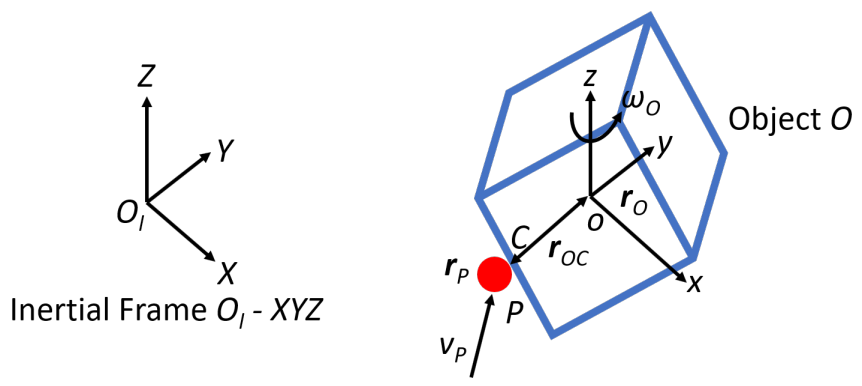

Figure 2.3: Single contact diagram of point $P$ with object $O$.

points to check at every time step of the simulation. Shan [41] outlines an additional step in contact detection when more contacting objects are involved, such as in the case of a net contacting the target.

The velocities and angular velocities of both objects after contact are given as:

$$
\begin{gathered}
\mathbf{v}_{p}^{+}=\mathbf{v}_{r}^{-}+\frac{j \mathbf{n}}{m_{P}} \\
\mathbf{v}_{O}^{+}=\mathbf{v}_{O}^{-}+\frac{j \mathbf{n}}{m_{O}} \\
\boldsymbol{\omega}_{O}^{+}=\boldsymbol{\omega}_{O}^{-}-\mathbf{I}_{O}^{-1}\left(\mathbf{r}_{O C}^{\times} j \mathbf{n}\right)
\end{gathered}
$$

where $j$ is the impulse generated from the contact, $m_{P}$ and $m_{O}$ are the masses of the mass point and target object, $\mathbf{I}_{O}$ is the moment of inertia of the target object $O$, and $\mathbf{r}_{O C}$ is the relative position of the contacting point of the mass and object, $C$, and the center of mass of the object, $O$. The superscripts ',' and '+' indicate the relative velocity of the two objects before and after the contact, respectively.

The coefficient of restitution is used to characterize the elasticity of the contact response between the two objects. The relative velocity change is expressed as:

$$
e=-\frac{v_{r}^{+}}{v_{r}^{-}}
$$

where $0 \leq e \leq 1$. These relative velocities are expressed as:

$$
v_{r}^{+}=\mathbf{n}\left(\dot{\mathbf{r}}_{P}^{+}-\dot{\mathbf{r}}_{O C}^{+}\right)
$$




$$
v_{r}^{+}=\mathbf{n}\left(\mathbf{v}_{P}^{+}-\left\{\mathbf{v}_{O}^{+}+\omega_{O}^{+\times} \mathbf{r}_{O C}\right\}\right)
$$

To calculate $j$, substitute Eqs. (2.32), (2.33), and (2.34) into Eq. (2.37) and use Eq. (2.35) to obtain:

$$
j=\frac{-(e+1) v_{r}^{-}}{\frac{1}{m_{P}}+\frac{1}{m_{O}}+\mathbf{n}\left(\left\{\mathbf{I}_{O}^{-1}\left(\mathbf{r}_{O C}^{\times} \mathbf{n}\right)\right\}^{\times} \mathbf{r}_{O C}\right)}
$$

Equation (2.38) is then used within Eqs. (2.32) and (2.33) to calculate the postcontact velocities of both objects.

In the case of the sub-tether configuration, multiple contacts may occur at once depending on the orientation of the target at the time of contact. Shan's [41] analytical solution to this problem is adapted for the sub-tether configuration and presented below.

The multiple contact problem is solved by decoupling each contact and establishing system equations to calculate the individual impulse on every contacting point. As stated in the conservation law of momentum, the linear and angular momentum will be conserved throughout the contact. They can be expressed as:

$$
\begin{gathered}
m_{O} \mathbf{v}_{O}^{-}+\sum\left(m_{b} \mathbf{v}_{i}^{-}\right)=m_{O} \mathbf{v}_{O}^{+}+\sum\left(m_{b} \mathbf{v}_{i}^{+}\right) \\
\mathbf{I}_{O}\left(\boldsymbol{\omega}_{O}^{+}-\boldsymbol{\omega}_{O}^{-}\right)=\sum\left(\mathbf{r}_{i}^{\times} j_{i} \mathbf{n}_{i}\right)
\end{gathered}
$$

where $\mathbf{v}_{i}$ is the velocity of the $i^{\text {th }}$ sub-tether bullet, $\mathbf{n}_{i}$ is the normal direction of the contact plane, $m_{b}$ is the mass of a single bullet node, and $j_{i}$ is the magnitude of the impulse of the $i^{\text {th }}$ contact.

The number of simultaneous contacts that can happen is defined as $k$. As constrained by the tether-only simulation, there are four sub-tethers on the novel tether configuration. Although every node of the tether can theoretically contact the target, in nominal cases the sub-tether bullets will contact the target first resulting in up to four simultaneous contacts occurring. The velocity and angular velocity of the object after contact with $k$ nodes is defined as: 


$$
\begin{aligned}
& \mathbf{v}_{O}^{+}=\mathbf{v}_{O}^{-}-\mathbf{N}^{T} \frac{\mathbf{j}}{m_{O}} \\
& \boldsymbol{\omega}_{O}^{+}=\boldsymbol{\omega}_{O}^{-}-\mathbf{I}_{O}^{-1} \sum_{r k}
\end{aligned}
$$

where the contact impulse vector, $\mathbf{j}$, is an undetermined vector based on the number of contacting points:

$$
\mathbf{j}=\left[j_{1}, j_{2}, \ldots, j_{k}\right]^{T}
$$

the normal vector, $\mathbf{N}$, is an undetermined vector based on the number of contacting points:

$$
\mathbf{N}=\left[n_{1}, n_{2}, \ldots, n_{k}\right]^{T}
$$

and $\sum_{r k}$ is defined as:

$$
\sum_{r k}=\sum_{i=1}^{i=k} \mathbf{r}_{i}^{\times} j_{i} \mathbf{n}_{i}
$$

Using the coefficient of restitution from Eq. (2.35) and the law of impulse conservation, the relative velocities after contact in the direction normal to the contact plane are derived as:

$$
\begin{gathered}
\left.-(e+1) v_{r i}^{-}=\mathbf{n}_{i} \frac{j_{i} \mathbf{n}_{i}}{m_{b}}+\mathbf{N}^{T} \frac{\mathbf{j}}{m_{O}}+\left(\mathbf{I}_{O}^{-1} \sum_{r k}\right)^{\times} \mathbf{r}_{i}\right] \\
\vdots \\
\left.-(e+1) v_{r k}^{-}=\mathbf{n}_{k} \frac{j_{k} \mathbf{n}_{k}}{m_{b}}+\mathbf{N}^{T} \frac{\mathbf{j}}{m_{O}}+\left(\mathbf{I}_{O}^{-1} \sum_{r k}\right)^{\times} \mathbf{r}_{k}\right]
\end{gathered}
$$

To simplify Eq. (2.46) two things are done. The first is the left side of the equation is simplified to:

$$
\mathbf{v}=-(e+1)\left[v_{r i}^{-}, v_{r i}^{-}, \ldots, v_{r k}^{-}\right]^{T}
$$


and the right side of the equation is reduced within the coefficient matrix $\mathbf{M}$ :

$$
\mathbf{M}=\left[\begin{array}{cccc}
\frac{1}{m_{b}}+\frac{1}{m_{O}}+\mathbf{n}_{1} \mathbf{I} \mathbf{C}_{11} & \frac{\mathbf{n}_{1} \mathbf{n}_{2}}{m_{O}}+\mathbf{n}_{1} \mathbf{I} \mathbf{C}_{21} & \ldots & \frac{\mathbf{n}_{1} \mathbf{n}_{k}}{m_{O}}+\mathbf{n}_{1} \mathbf{I} \mathbf{C}_{k 1} \\
\frac{\mathbf{n}_{2} \mathbf{n}_{2}}{m_{O}}+\mathbf{n}_{1} \mathbf{I} \mathbf{C}_{12} & \frac{1}{m_{b}}+\frac{1}{m_{O}}+\mathbf{n}_{2} \mathbf{I} \mathbf{C}_{22} & \ldots & \frac{\mathbf{n}_{2} \mathbf{n}_{k}}{m_{O}}+\mathbf{n}_{2} \mathbf{I} \mathbf{C}_{k 2} \\
\vdots & \vdots & \ddots & \vdots \\
\frac{\mathbf{n}_{k} \mathbf{n}_{1}}{m_{O}}+\mathbf{n}_{k} \mathbf{I} \mathbf{C}_{1 k} & \cdots & \cdots & \frac{1}{m_{b}}+\frac{1}{m_{O}}+\mathbf{n}_{k} \mathbf{I} \mathbf{C}_{k k}
\end{array}\right]
$$

where

$$
\mathbf{I C}_{i k}=\left(\mathbf{I}_{O}^{-1}\left\{\mathbf{r}_{i}^{\times} \mathbf{n}_{i}\right\}\right)^{\times} \mathbf{r}_{k}
$$

Using these simplifications, Eq. (2.46) is rewritten as:

$$
\mathbf{v}=\mathbf{M j}
$$

and the impulse of each contacting point can be calculated as:

$$
\mathbf{j}=\mathbf{M}^{-1} \mathbf{v}
$$

In the same vein as single contact, Eq. (2.51) can be used in Eqs. (2.41) and (2.42) to calculate the velocities after contact.

In addition to the impulse-based model, an additional penalty-based model was implemented by Shan [41]. In this model, contact forces are expressed as continuous functions of the penetrations between contacting bodies. It could not be implemented within this method as the penalty-based method cannot work with discrete models that assume the impact process is instantaneous. For this reason, it has been omitted in this work. In future work building upon this thesis by modeling a net attached to the end of the sub-tether configuration, it is recommended that this additional contact dynamics model be implemented. 


\subsubsection{Post-Capture Dynamics Formulation - Spring-Damper Tether Model}

Following Hovell's [46] work, the post-capture phase tethers are modeled as a single massless spring-damper element between the respective spacecraft body and junction. Torsion and bending effects are ignored. External forces are only generated while the tether elements are in tension, and they are applied to each connected mass.

Defining the $i^{\text {th }}$ sub-tether under analysis by the vector $\vec{L}_{i}$, in $\mathcal{F}_{I}$ has components of:

$$
\mathbf{L}_{i}=\mathbf{r}_{n_{i}}-\mathbf{r}_{t}-\mathbf{C}_{\mathrm{BI}}(\mathbf{q})^{\mathrm{T}} \mathbf{a t t}_{i}, \quad \forall i=1, \ldots, 4
$$

where $\mathbf{a t t}_{i}$ is the attachment point of the $i^{\text {th }}$ sub-tether relative to the center of mass of the target in $\mathcal{F}_{B}$.

By treating the linear spring and damper in parallel, the resultant tensile force magnitude developed by the $i^{\text {th }}$ sub-tether is:

$$
F_{i}= \begin{cases}k\left(\left\|\mathbf{L}_{i}\right\|-L_{0}\right)+c\left[\mathbf{v}_{n_{i}}-\left(\mathbf{v}_{t}+\mathbf{C}_{\mathrm{BI}}(\mathbf{q})^{\mathrm{T}} \boldsymbol{\omega}^{\times} \mathbf{a t t}_{i}\right)\right]^{\mathrm{T}} \frac{\mathbf{L}_{i}}{\left\|\mathbf{L}_{i}\right\|}, & \text { for }\left\|\mathbf{L}_{i}\right\|-L_{0}>0 \\ 0, & \text { otherwise }\end{cases}
$$

where $\mathbf{v}_{t}$ and $\mathbf{v}_{n_{i}}$ are the velocity components of the target and junction in $\mathcal{F}_{I}$, respectively. The tether material is assumed to have a constant spring constant, $k$, and damping coefficient, $c$, calculated as discussed in Eqs. 2.17 and 2.18, respectively.

\subsubsection{Equations of Motion}

The equations of motion of the chaser, target, and tether can be broken down into two phases: deployment of the tether, and the detumbling of the TSS.

To model the motion, all forces contributing to translational motion for each body are included in Eq. (2.13). As a result of the chaser and tether nodes modeled as point masses, rotational motion is only modeled for the target using Eq. (2.4).

During the tether deployment phase the resulting net force on the target in $\mathcal{F}_{I}$ is 
only due to gravitational effects. It is given by:

$$
m_{t} \frac{\mathrm{d} \dot{\mathbf{r}}_{t}}{\mathrm{~d} t}=\mathbf{F}_{t}=\mathbf{F}_{g}=-\frac{\mu m_{t}}{\left\|\mathbf{r}_{t}\right\|^{3}} \mathbf{r}_{t}
$$

Similarly, torque imparted on the target is calculated only through contributions by gravity-gradient effects defined in $\mathcal{F}_{B}$

$$
\boldsymbol{\tau}_{t}=\boldsymbol{\tau}_{g}=\frac{3 \mu}{\left\|\mathbf{r}_{t}^{b}\right\|^{5}} \mathbf{r}_{t}^{b \times} \mathbf{J r}_{t}^{b}
$$

The chaser spacecraft will be affected by gravitational effects and an impulse force, $\mathbf{F}_{\text {impulse }}$, that occurs when the tether is deployed. In cases, the chaser will also be affected by the addition of its own thrust force, $\mathbf{F}_{\text {thrust }}$. Although these forces are conditional, together they result in:

$$
m_{c} \frac{\mathrm{d} \dot{\mathbf{r}}_{c}}{\mathrm{~d} t}=\mathbf{F}_{c}=\mathbf{F}_{g}-\mathbf{F}_{\text {impulse }}+\mathbf{F}_{\text {thrust }}=-\frac{\mu m_{c}}{\left\|\mathbf{r}_{c}\right\|^{3}} \mathbf{r}_{c}-\sum_{i=1}^{4} F_{\text {impulse } i} \frac{\mathbf{r}_{b}}{\left\|\mathbf{r}_{b}\right\|}+\mathbf{F}_{\text {thrust }}
$$

A given tether node, denoted by the subscript $i$, experiences similar forces to the chaser before they are deployed from its surface as a result of being pulled by the proceeding node in the tether. A bullet will experience an additional one-time impulse force. These forces are given by the conditions:

$$
m_{n_{i}} \frac{\mathrm{d} \dot{n}_{n_{i}}}{\mathrm{~d} t}=\mathbf{F}_{n_{i}}= \begin{cases}\mathbf{F}_{c}, & \text { when } i \text { is attached to the chaser } \\ \mathbf{F}_{g_{i}}+\mathbf{F}_{i i+1}+\mathbf{F}_{i i-1}, & \text { when } i \text { is a tether node in flight } \\ \mathbf{F}_{g_{i}}+\sum_{k=1}^{4} \mathbf{F}_{i_{k_{1}}}+\mathbf{F}_{\text {imend }}, & \text { when } i \text { is the junction node in flight } \\ \mathbf{F}_{g_{i}}+\mathbf{F}_{i i-1}+\mathbf{F}_{\text {impulse }}, & \text { when } i \text { is a bullet node in flight }\end{cases}
$$

where the subscript ' $s$ ' denotes the sub-tether, ' $m$ ' denotes the main tether, and ' ${ }_{i-1}$ ' and ' ${ }_{i+1}$ ' denote the node before and after node $i$ of the tether, respectively.

During the contact phase the tether bullets and target post-contact velocities are calculated and applied at the first step of the post-capture phase. Practically, the tether bullets are much smaller in mass than the target and the impulse imparted 
is negligible. Omitting this step in simulation has no consequential effects for the simulation. However, if a net at the end of the sub-tethers were to be included this step would be necessary.

During the post-capture stabilization phase the resulting net force on the target in $\mathcal{F}_{I}$ is due to the tension forces generated in the four sub-tethers and the gravitational effects. It is given by:

$$
m_{t} \frac{\mathrm{d} \dot{\mathbf{r}}_{t}}{\mathrm{~d} t}=\mathbf{F}_{t}=\mathbf{F}_{s}+\mathbf{F}_{g}=\sum_{i=1}^{4} F_{i} \frac{\mathbf{L}_{i}}{\left\|\mathbf{L}_{i}\right\|}-\frac{\mu m_{t}}{\left\|\mathbf{r}_{t}\right\|^{3}} \mathbf{r}_{t}
$$

In cases, the chaser spacecraft will be affected by the addition of its own thrust force, $\mathbf{F}_{\text {thrust }}$. This results in:

$$
m_{c} \frac{\mathrm{d} \dot{\mathbf{r}}_{c}}{\mathrm{~d} t}=\mathbf{F}_{c}=-\mathbf{F}_{m}+\mathbf{F}_{g}+\mathbf{F}_{\text {thrust }}=-F_{m} \frac{\mathbf{L}_{m}}{\left\|\mathbf{L}_{m}\right\|}-\frac{\mu m_{c}}{\left\|\mathbf{r}_{c}\right\|^{3}} \mathbf{r}_{c}+\mathbf{F}_{\text {thrust }}
$$

where, $F_{m}$ denotes the force magnitude present in the main tether and $\mathbf{L}_{m}$ denotes its vector components. The junction experiences similar forces:

$$
m_{j} \frac{\mathrm{d} \dot{\mathbf{r}}_{j}}{\mathrm{~d} t}=\mathbf{F}_{j}=\mathbf{F}_{m}-\mathbf{F}_{s}+\mathbf{F}_{g}=F_{m} \frac{\mathbf{L}_{m}}{\left\|\mathbf{L}_{m}\right\|}-\sum_{i=1}^{4} F_{i} \frac{\mathbf{L}_{i}}{\left\|\mathbf{L}_{i}\right\|}-\frac{\mu m_{j}}{\left\|\mathbf{r}_{j}\right\|^{3}} \mathbf{r}_{n_{i}}
$$

Torque imparted on the target is calculated as the sum of the four sub-tether contributions and the gravity-gradient effects defined in $\mathcal{F}_{B}$.

$$
\boldsymbol{\tau}_{t}=\boldsymbol{\tau}_{s}+\boldsymbol{\tau}_{g}=\mathbf{C}_{\mathrm{BI}}(\mathbf{q}) \sum_{i=1}^{4} F_{i} \frac{\mathbf{a}_{i}^{\times} \mathbf{L}_{i}}{\left\|\mathbf{L}_{i}\right\|}+\frac{3 \mu}{\left\|\mathbf{r}_{t}^{b}\right\|^{5}} \mathbf{r}_{t}^{b \times} \mathbf{J r}_{t}^{b}
$$

To simulate the dynamics, Eqs. (2.58) to (2.61) are solved numerically with Eqs. (2.4) and (2.13).

\subsection{On-Orbit Numerical Simulations}

The equations of motion and tether numerical models presented in Sec. 2.2 are used to build upon Hovell and Ulrich's [11] work to examine the deployment dynamics of the novel tether configuration and create a complete end-to-end tether deployment 
and target detumbling scenario. This section presents the orbital simulation of the complete capture scenario.

The target is modeled as a rectangular prism and the chaser as a point mass. Two separate simulations are run over one orbital period and combined together in order to achieve the full deployment and capture scenario. First, the tether deployment is simulated up until the point of contact between the tether bullets and the target, and secondly, with the tethers attached to the target. The initial orbital elements of the target are defined in Table 2.1.

Table 2.1: Initial orbital elements of the on-orbit complete capture simulation.

\begin{tabular}{cc}
\hline \hline Orbital Element & Value \\
\hline Semi-major axis, $a$ & $6871 \mathrm{~km}$ \\
Eccentricity, $e$ & 0.001 \\
Right ascension of & $20 \mathrm{deg}$ \\
the ascending node, $\Omega$ & \\
Inclination, $i$ & $60 \mathrm{deg}$ \\
Argument of perigee, $\omega$ & $90 \mathrm{deg}$ \\
True anomaly, $\theta$ & $60 \mathrm{deg}$ \\
\hline \hline
\end{tabular}

For use in the simulation, the target and chaser initial positions, $\mathbf{R}$, and velocities, $\mathbf{V}$, in $\mathcal{F}_{I}$ are required. These are calculated using the orbital elements. First, the magnitude of the radius vector, $R$, is calculated:

$$
R=\frac{a\left(1-e^{2}\right)}{1+e \cos \theta}
$$

then using this magnitude, the position and velocity vector components in the perifocal reference frame, $\mathcal{F}_{p}$, are calculated as:

$$
\mathbf{R}_{p}=\left[\begin{array}{c}
R \cos \theta \\
R \sin \theta \\
0
\end{array}\right]
$$




$$
\mathbf{V}_{p}=\left[\begin{array}{c}
-\sqrt{\frac{\mu}{a\left(1-e^{2}\right)}} \sin \theta \\
\sqrt{\frac{\mu}{a\left(1-e^{2}\right)}}(e+\cos \theta) \\
0
\end{array}\right]
$$

where $\mu$ is the gravitational parameter of the Earth. The $\mathbf{R}_{p}$ and $\mathbf{V}_{p}$ vectors are then rotated into $\mathcal{F}_{I}$ through the rotation matrix:

$\mathbf{C}_{I P}=\left[\begin{array}{ccc}\cos \Omega \cos \omega-\sin \Omega \cos i \sin \omega & -\cos \Omega \sin \omega-\sin \Omega \cos i \cos \omega & \sin \Omega \sin i \\ \sin \Omega \cos \omega+\cos \Omega \cos i \sin \omega & -\sin \Omega \sin \omega+\cos \Omega \cos i \cos \omega & -\cos \Omega \sin i \\ \sin i \sin \omega & \sin i \cos \omega & \cos i\end{array}\right]$

which is used to obtain $\mathbf{R}_{I}$ and $\mathbf{V}_{I}$ of the target:

$$
\begin{gathered}
\mathbf{R}_{I_{t}}=\mathbf{C}_{I P} \mathbf{R}_{P}=\left[\begin{array}{c}
-6176 \\
-421 \\
2974
\end{array}\right] \mathrm{km} \\
\mathbf{V}_{I_{t}}=\mathbf{C}_{I P} \mathbf{V}_{P}=\left[\begin{array}{c}
-2.46 \\
-4.40 \\
-5.71
\end{array}\right] \mathrm{km} / \mathrm{s}
\end{gathered}
$$

The initial position and velocity vectors of the chaser spacecraft are similarly calculated, however it's position trails the target by $30 \mathrm{~m}$ in the flight direction.

The simulations are performed using an Adams integration scheme over 5668 seconds (one orbit). It uses a 0.1 second time step for the tether deployment portion, and a 1 second time step for the post-capture stabilization portion. The use of different time steps is done to better visualize the deployment phase of the simulation, while advancing it at a quicker rate during the detumbling phase. The tether bullets are deployed each at a unique normalized vector, $\mathbf{d}_{i}$, so that they radiate outwards from the chaser. The subscript $i$ denotes the bullet number. Simulation parameters are found in Table 2.2 .

Upon contact between the tether and target, the simulation is stopped and the final time step values of the deployment simulation are used as the initial conditions 
Table 2.2: Initial conditions and parameters of the tether deployment simulation.

\begin{tabular}{cccccc}
\hline \hline Parameter & Value & Parameter & Value & Parameter & Value \\
\hline$J_{x x}, \mathrm{~kg} \cdot \mathrm{m}^{2}$ & 15000 & $m_{t}, \mathrm{~kg}$ & 3000 & $\mathbf{d}_{1}$ & {$[0.9182,0.0193,0.3957]$} \\
$J_{y y}, \mathrm{~kg} \cdot \mathrm{m}^{2}$ & 3000 & $m_{c}, \mathrm{~kg}$ & 500 & $\mathbf{d}_{2}$ & {$[0.9318,0.0196,0.3625]$} \\
$J_{z z}, \mathrm{~kg} \cdot \mathrm{m}^{2}$ & 15000 & $m_{n}, \mathrm{~kg}$ & 1 & $\mathbf{d}_{3}$ & {$[0.9182,-0.0193,0.3957]$} \\
$k, \frac{\mathrm{N}}{\mathrm{m}}$ & 3150 & $m_{b}, \mathrm{~kg}$ & 5 & $\mathbf{d}_{4}$ & {$[0.9318,-0.0196,0.3625]$} \\
$c, \frac{\mathrm{Ns}}{\mathrm{m}}$ & 16 & $m_{j}, \mathrm{~kg}$ & 10 & Target size, $\mathrm{m}$ & {$[1.25,1.75,1.25]$} \\
$L_{\text {main }}, \mathrm{m}$ & 15.0 & $L_{\text {sub }}, \mathrm{m}$ & 15.2 & $v_{b}, \frac{\mathrm{m}}{\mathrm{s}}$ & 0.08 \\
$\beta$ & 0.3025 & $\gamma$ & 0.6 & \# nodes/tether & 5 \\
\hline \hline
\end{tabular}

of the detumbling simulation. It is run for the remainder of the 5668 second orbital period. Initial condition values of the detumbling simulation are both the chaser and target positions and velocities, the targets attitude, angular rate, and the four contact points of the tether bullets with the target in $\mathcal{F}_{B}$. The bullet contact points with the target were found to be consistent between both the spring-damper and ANCF simulations with negligible differences. As a result, one set of attachment points is used. The initial conditions used for the post-capture detumbling simulation are in Table 2.3.

Table 2.3: Initial conditions and parameters of the on-obrit post-capture simulation.

\begin{tabular}{cc}
\hline \hline Initial Condition & Value \\
\hline Target attitude, $\mathbf{q}$ & {$[0.2113,-0.4532,0.8627,0.0755]$} \\
Target angular rate, $\boldsymbol{\omega}$ & {$[-0.0096 .0 .0252 .0 .0341] \mathrm{rad}$} \\
Target position, $\mathbf{R}_{I_{t}}$ & {$[-6547,-1970,6720] \mathrm{km}$} \\
Target velocity, $\mathbf{V}_{I_{t}}$ & {$[0.47,-3.85,-6.55] \mathrm{km} / \mathrm{s}$} \\
Chaser position, $\mathbf{R}_{I_{c}}$ & {$[-6547,-1970,6720] \mathrm{km}$} \\
Chaser velocity, $\mathbf{V}_{I_{c}}$ & {$[0.47,-3.85,-6.55] \mathrm{km} / \mathrm{s}$} \\
Tether junction position, $\mathbf{R}_{I_{j}}$ & {$[-6547,-1970,6720] \mathrm{km}$} \\
Tether junction velocity, $\mathbf{V}_{I_{j}}$ & {$[0.47,-3.85,-6.55] \mathrm{km} / \mathrm{s}$} \\
Bullet 1 attachment, att & {$[0.625,-0.875,0.000] \mathrm{m}$} \\
Bullet 2 attachment, att $_{2}$ & {$[0.000,-0.875,0.625] \mathrm{m}$} \\
Bullet 3 attachment, att $_{3}$ & {$[-0.625,-0.875,0.000] \mathrm{m}$} \\
Bullet 4 attachment, att $_{4}$ & {$[0.000,-0.875,-0.625] \mathrm{m}$} \\
\hline \hline
\end{tabular}

A modified version of the first detumbling case examined by Hovell [46] is presented 
in this section. In this modified case, the tether does not initially connect the chaser and target. Instead, it is deployed from the chaser and stretches to it's maximum unstretched length, at which point contact between the target and tether is made and the detumbling scenario begins. Once detumbling, the chaser continually thrusts in the retrograde direction with a force of $20 \mathrm{~N}$ to maintain tension on the tether. The initial tether angle of $\pi / 6$ radians and single spin axis angular rate that Hovell [46] used for his first detumbling case are omitted in order to maintain the attitude and angular rates of the target at the time of instantaneous capture.

Figures. 2.4(a) to 2.4(h) depict the novel tether configuration deployment using the spring-damper model, and Fig. 2.5(a) to 2.5(h) depict the novel tether configuration deployment using the ANCF model. The tether deployment using both models takes place over 370 seconds. Fig. 2.6 shows the immediate TSS configuration post-capture. All on-orbit simulation figures are displayed using a fixed inertial frame. A video of the full capture scenario can be accessed at https://tinyurl.com/yy9faazm.

Figure 2.7 shows the results of the post-capture stabilization process given the initial conditions found in Table 2.3 over the remainder of the orbital period once the target has been captured. Fig. 2.7(a) shows the targets angular rates settling, Fig. 2.7(b) shows the tether equilibrium angle approaching zero, and Fig. 2.7(c) shows the tether elongation diminishing over time. These results are consistent with Hovell's [46] findings, and demonstrate the passive stabilization properties of the subtether configuration are still present in a full capture scenario. From this orbital simulation, it is concluded that the novel tether configuration is capable of deploying nominally in an orbital environment, contacting a tumbling target, and passively reducing it's angular velocity over time. 


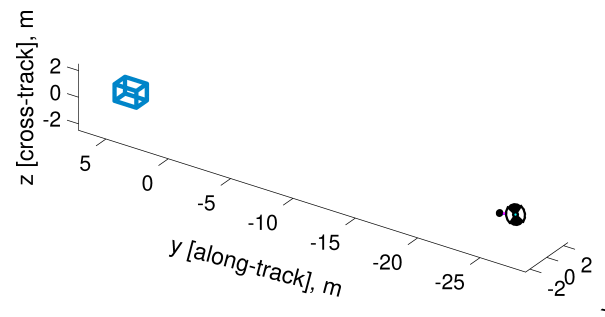

(a) Spring-damper model tether deployment initial configuration

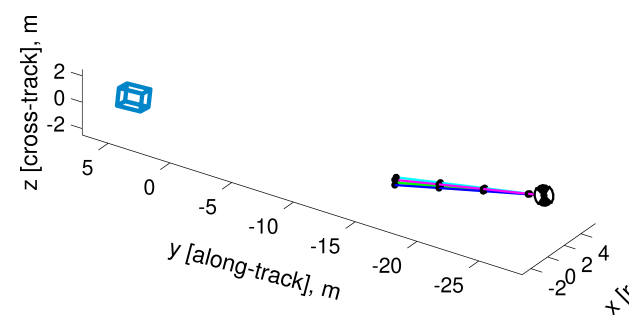

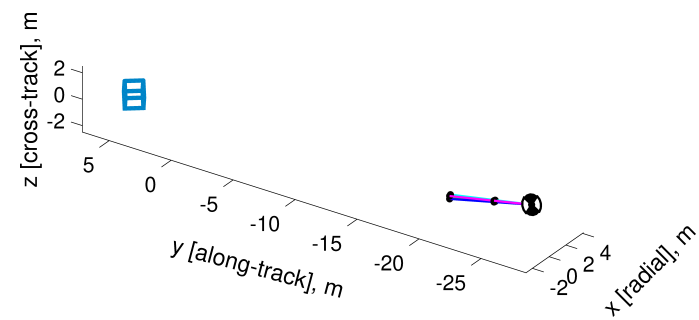

(b) Spring-damper model tether deployment $1 \%$ through orbit

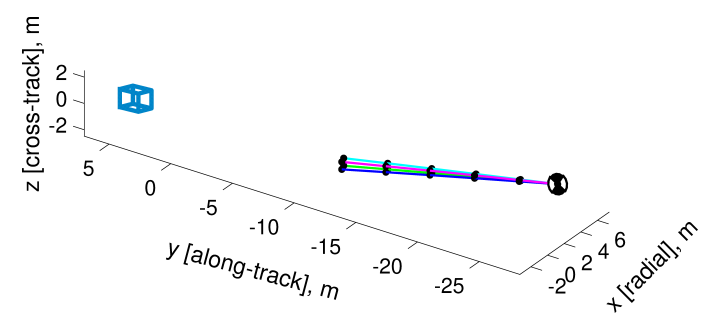

(c) Spring-damper model tether deployment (d) Spring-damper model tether deployment $2 \%$ through orbit

$3 \%$ through orbit
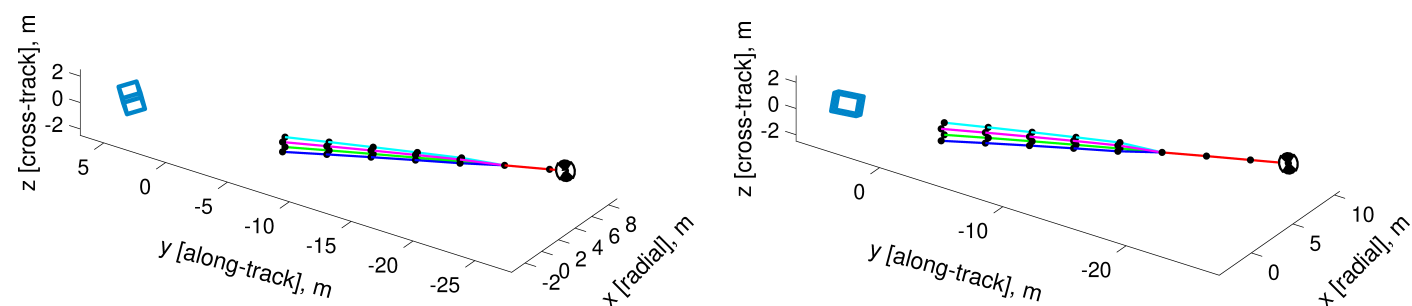

(e) Spring-damper model tether deployment (f) Spring-damper model tether deployment $4 \%$ through orbit

$5 \%$ through orbit
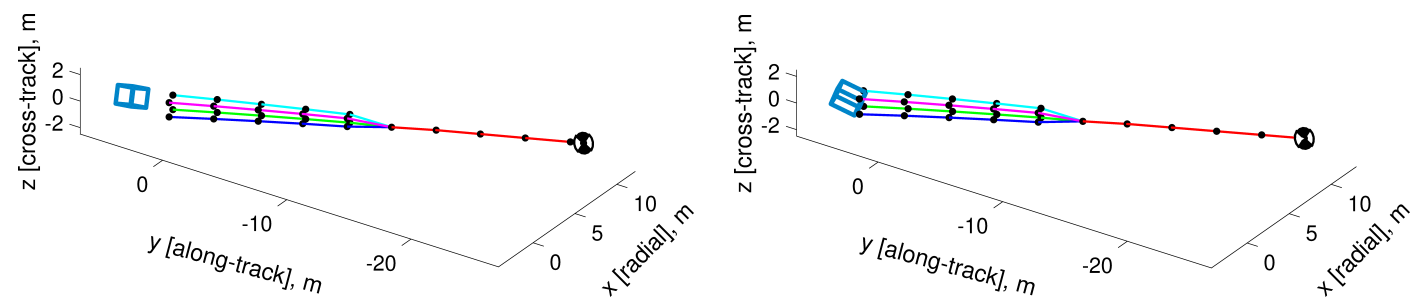

(g) Spring-damper model tether deployment (h) Spring-damper model tether deployment $6 \%$ through orbit

$6.5 \%$ through orbit, contacting the target

Figure 2.4: Novel tether configuration deployment in an orbital environment using the massless spring-damper model. 


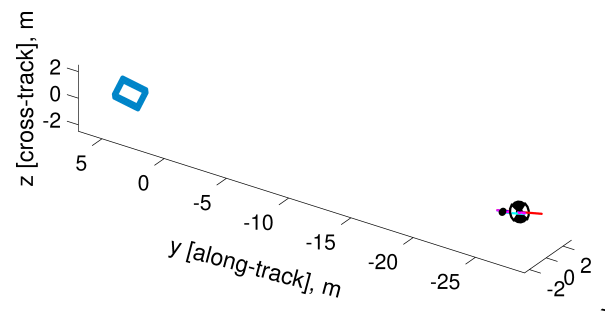

(a) ANCF model tether deployment initial configuration

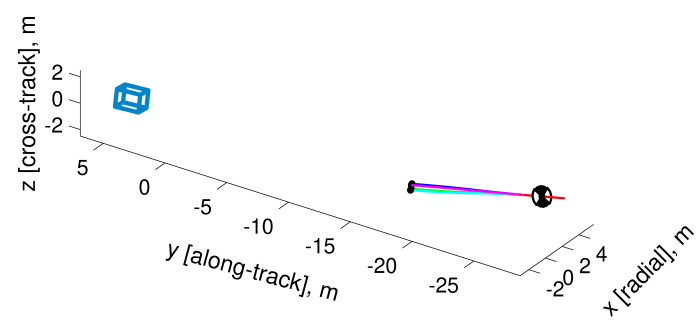

(c) ANCF model tether deployment $2 \%$ through orbit

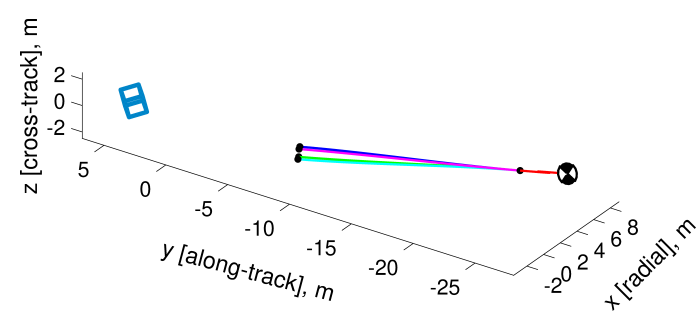

(e) ANCF model tether deployment $4 \%$ through orbit

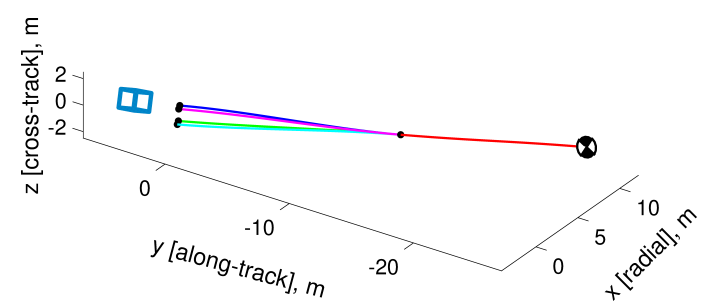

(g) ANCF model tether deployment $6 \%$ through orbit

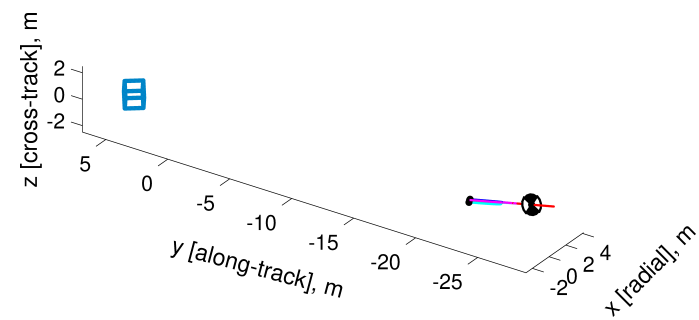

(b) ANCF model tether deployment $1 \%$ through orbit

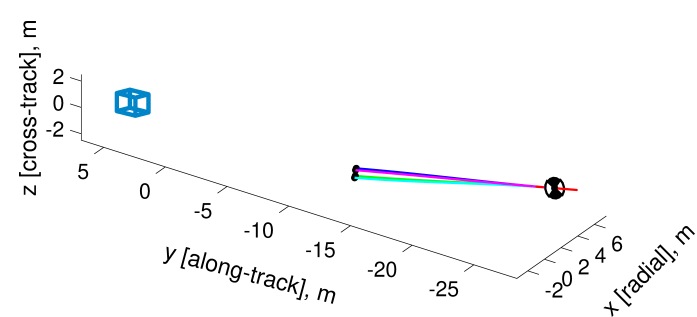

(d) ANCF model tether deployment $3 \%$ through orbit

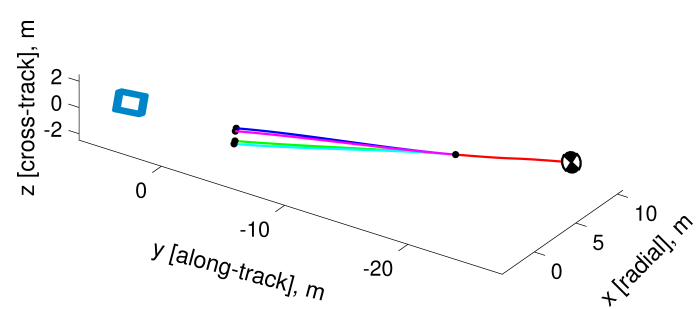

(f) ANCF model tether deployment 5\% through orbit

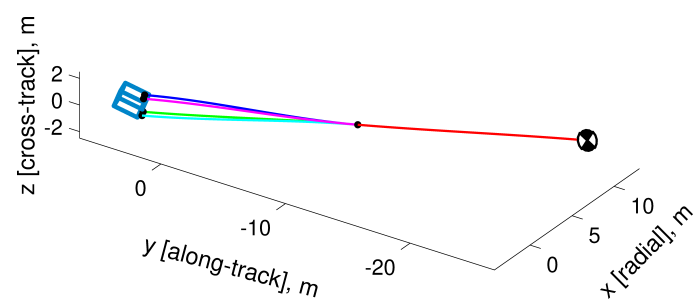

\% (h) ANCF model tether deployment $6.5 \%$ through orbit, contacting the target

Figure 2.5: Novel tether configuration deployment in an orbital environment using the ANCF model. 


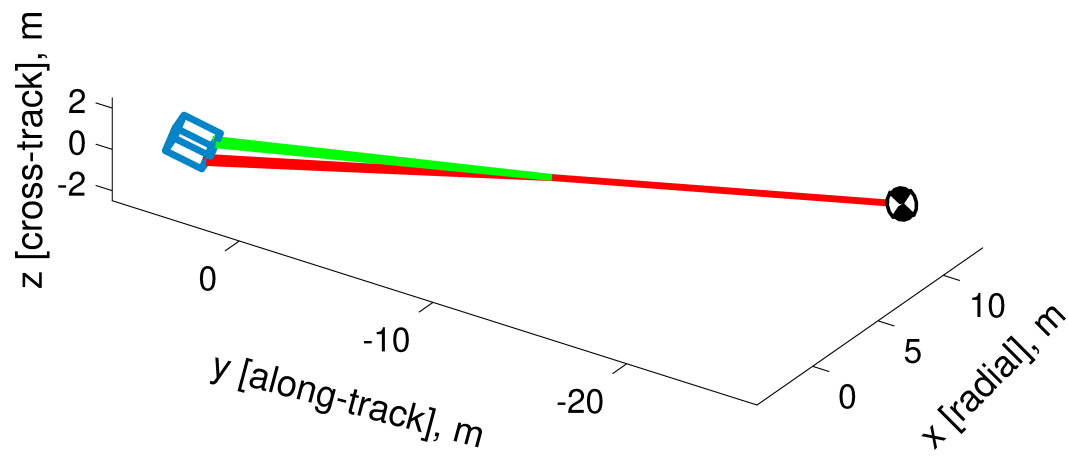

Figure 2.6: Initial position of the TSS post-capture. 


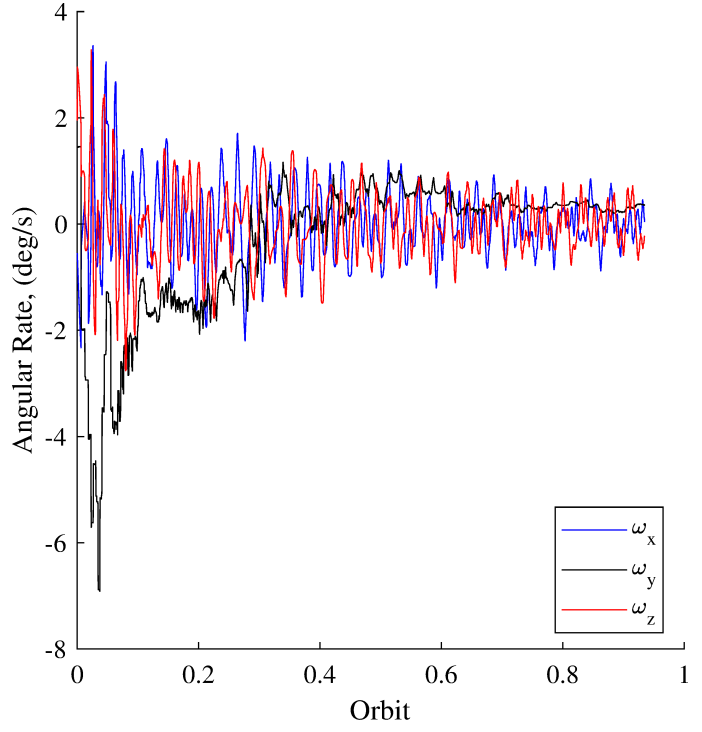

(a) Target angular rates

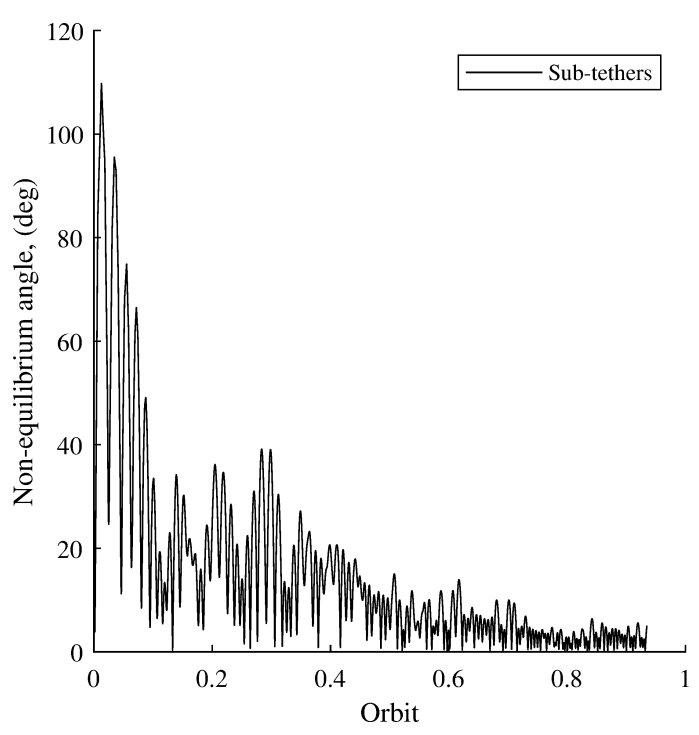

(b) Tether angle

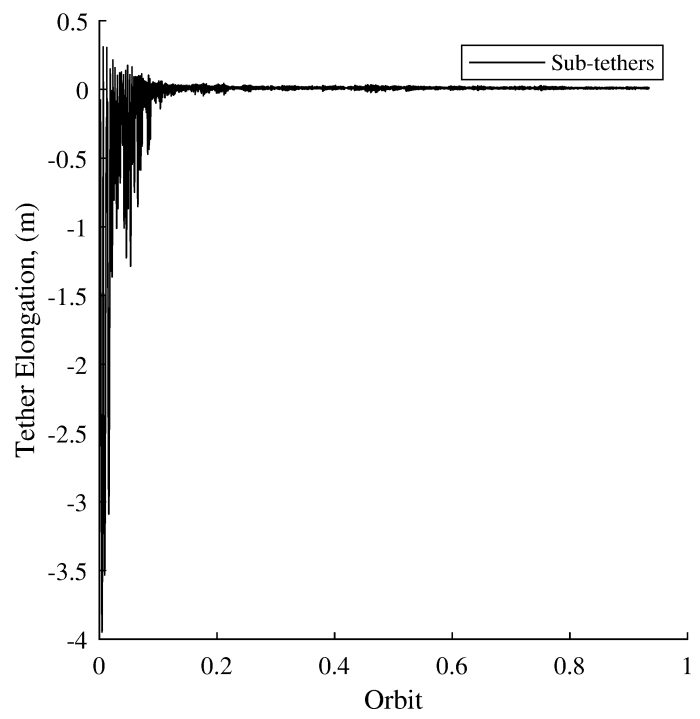

(c) Tether elongation

Figure 2.7: Detumbling motion of the tethered spacecraft system. 


\section{Chapter 3}

\section{Experimental Setup}

In order to experimentally validate the tether deployment and complete capture simulations, a tether deployment apparatus was constructed for use with Carleton University's Spacecraft Proximity Operations Testbed (SPOT) which is housed within the Spacecraft Robotics and Control Laboratory (SRCL). This chapter outlines the SPOT facility, the tether deployment apparatus, and accompanying laboratory components.

\subsection{Experimental Facility}

The SRCL is located at Carleton University in Ottawa, Ontario, Canada. It houses the SPOT, which consists of a smooth granite table mounted on a steel frame. Two identical platforms are used on this table to simulate spacecraft. They float through the use of air bearings which expel air downwards, creating a thin film of air between themselves and the table. This floating process is near friction-free and allows the platforms to mimic deep space movement within a plane of motion. Planar translational and rotational motion is done using eight air jets located on all four sides of each platform.

Both platforms are modular and can be easily modified to accommodate various experimental scenarios. The platform structures house three main levels each with a specific purpose. The bus is located on the first level of the platform. It contains the air tank, the flotation system, and the propulsion system. The avionics level is located in the middle. It contains the power, battery, computer, control panel, and reaction wheel systems. Lastly, the top level houses the payload. The two available payloads are a computer vision system and the tether deployment system outlined in Sec. 3.2.1. Additional exterior add-on systems are also available, such as a robotic arm or an electromagnetic docking system. 
MATLAB and Simulink software is used to define the platforms movement logic and accompanying control algorithms. This allows a user to create an experimental scenario that the platforms can execute. The scenario is compiled on the ground station computer and transmitted to the platforms prior to running an experiment. Each platform uses a Raspberry Pi 3 as an on-board computer to operate. They communicate wireless with the ground station computer while executing experiments to receive data and commands. Ground truth infrastructure, called the PhaseSpace system, records the platform's positions and attitudes during experiments using four LED's on each platform and transmits the data to the platforms in real-time for use in closed-loop control systems.

An overview of the SRCL and SPOT as of September 2019 is shown in Fig. 3.1. It shows the granite table with its protective cover on, the ground station computer and emergency stop button, the two spacecraft platforms, and PhaseSpace cameras mounted along the walls. Figure 3.2 highlights the two spacecraft platforms.

\subsection{Experimental Apparatus}

The experimental apparatus used in the tether deployment and complete capture scenario experiments is comprised of several different components. The tether deployment payload of the platform is the largest and most complex component, while the accompanying magnetic impact surface, bullets, and tether are smaller, yet equally important to the experiment.

The concept of the tether deployment mechanism is similar to that which would be found on an orbital debris removal mission. Due to the planar limitations of the laboratory it is not feasible to experimentally validate the novel tether configuration

while attached to a net. For this reason the bullets are attached directly to the ends of the tethers, and the tethers themselves are deployed rather than being pulled along by a deployed net. The connection method of metal-tipped bullets and a magnetic contact surface is also unrealistic for net capture as the net would wrap around the target and not stick to the surface with as much force. However, due to the planar limitations of the SPOT this method was deemed the most appropriate approach for validating the motion of the tethers during deployment. 


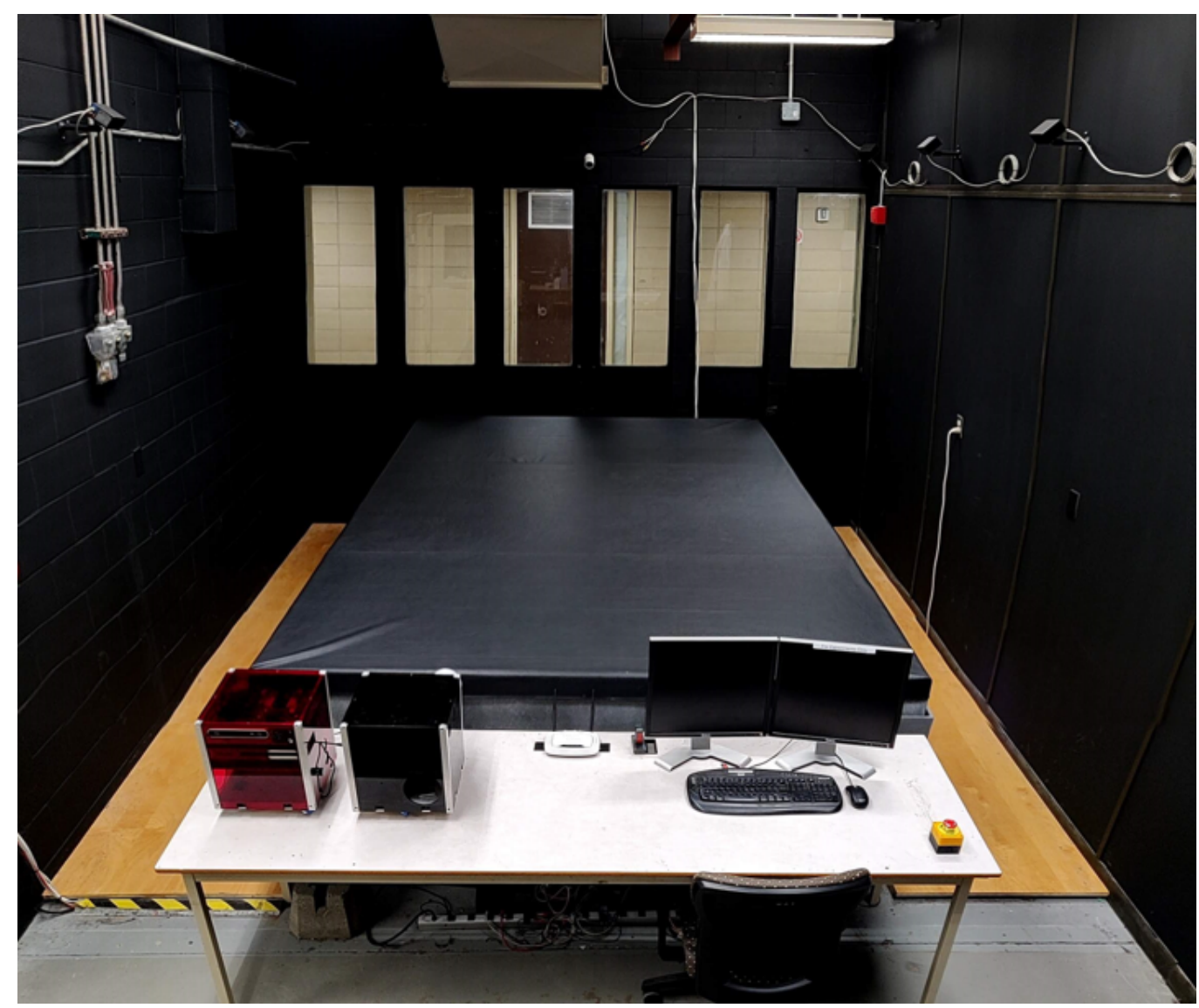

Figure 3.1: Overview of the Spacecraft Proximity Operations Testbed.

\subsubsection{Tether Deployment Apparatus}

The experimental apparatus is designed to launch two end masses, called bullets, which are connected to the tether from one platform to the other. Figure 3.3 shows and overview of this system. It uses compressed air to propel the bullets. The air begins in a 3000 psi tank with a built-in regulator that lowers the output pressure to 800 psi. The tank is connected to a high-pressure regulator through an air tank adapter and steel piping. When the turn-knob on the air tank is opened air is allowed to flow into the system. The high-pressure regulator allows air to pass through within a range of 0-500 psi, and is controlled through the use of a turn-knob. The system nominally operates at just below 250 psi. System pressure is monitored by an analog pressure gauge downstream of the high-pressure regulator. A 250 psi safety release valve is located between the high-pressure regulator and the normally closed solenoid 


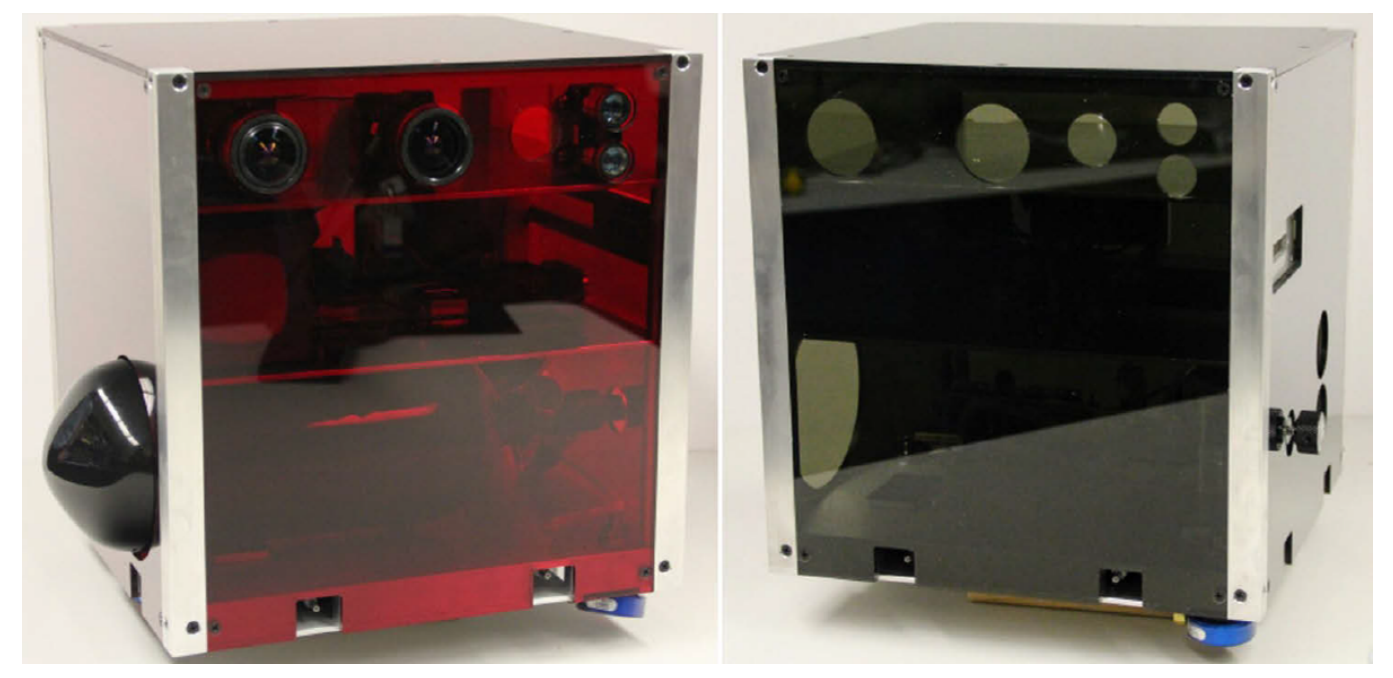

Figure 3.2: SPOT platforms.

valve connected to a small circuit on a breadboard. The solenoid valve has a burst pressure of $500 \mathrm{psi}$, and in the event that the high-pressure regulator fails the safety release valve will vent the system air to protect the solenoid valve from breaking. At the appropriate time during an experiment the platform Raspberry Pi will send a signal to the transistor on the solenoid valve signal control breadboard, closing the circuit and allowing power to flow to the solenoid valve, opening it. Air in the system will then continue to flow, exiting the system and propelling the bullets to their target.

\subsubsection{Tether Bullet}

The tether bullets are loosely modeled after harpoons. They are 3D printed using fused deposition modeling and an acrylonitrile butadiene styrene plastic filament. 3D printing is used as it allows for a lightweight and durable final product. The tether bullet is designed to interface with the tether deployment payload by sliding over the copper tubing of the system. This gives the bullets initial stability in their trajectory as they are propelled forward by the air, similar to the function of a cannon barrel. The bullets have a small metal tip in order to stick to the magnets located on the target impact panel. The tether is attached to the outer wall at the base of the bullet by a small strip of adhesive tape. A singular bullet can be seen in Fig. 3.4. Both 


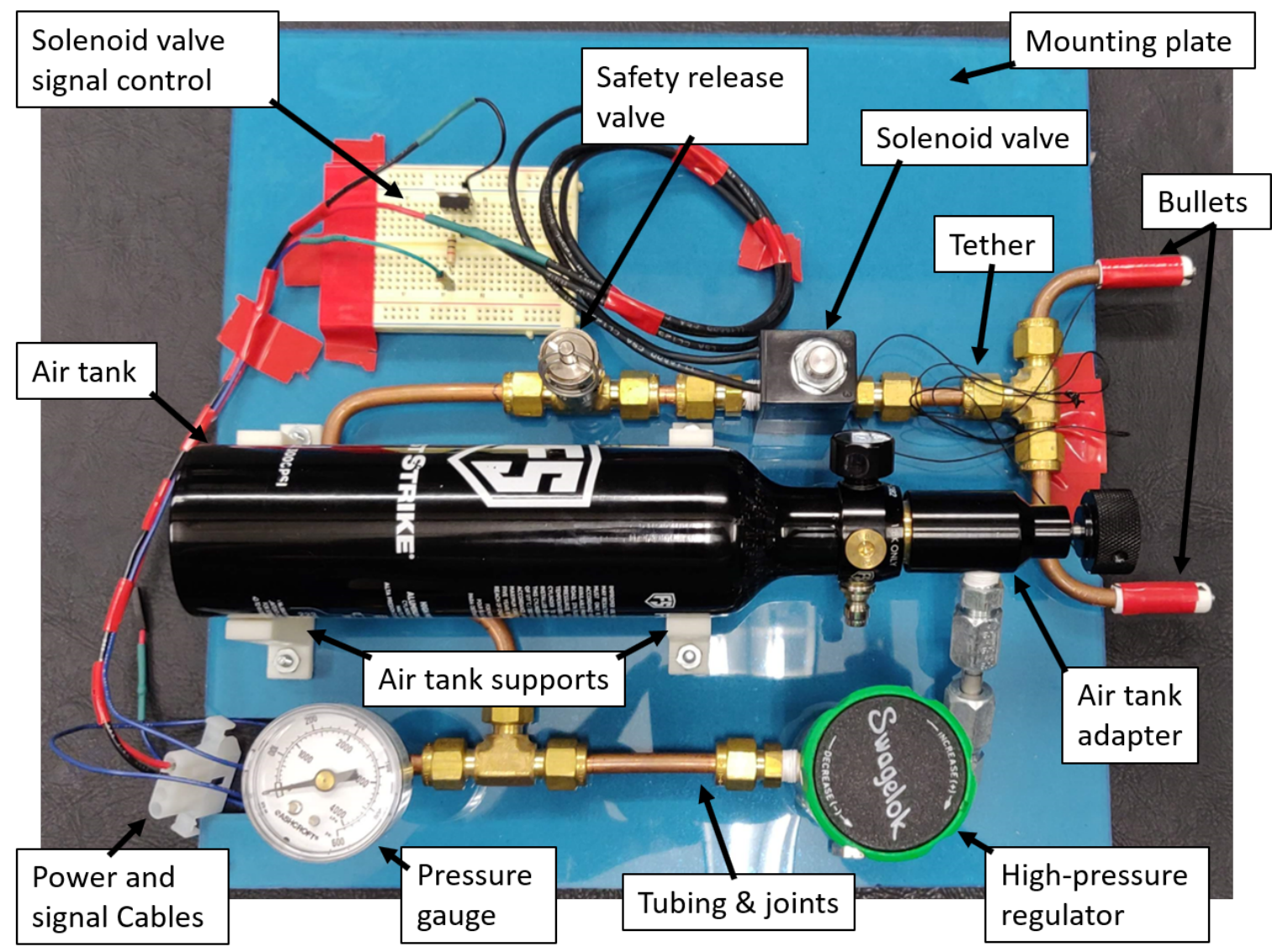

Figure 3.3: Tether Deployment Payload.

bullets used in the experiment are identical.

It is noted that unlike the simulations, the tether bullets are used in an environment with air. Early iterations of the bullets included fins for experimental use. They were intended to stabilize the bullets in flight due to the encountered air resistance, and move the center of mass of the bullet towards the rear. The addition of fins used for ground testing has been seen in other cases of dedicated space debris harpoons [56]. Ultimately, the fins were determined to not be necessary as they added too much mass to the overall weight of the bullet, and were too small to effectively stabilize it while in flight. 


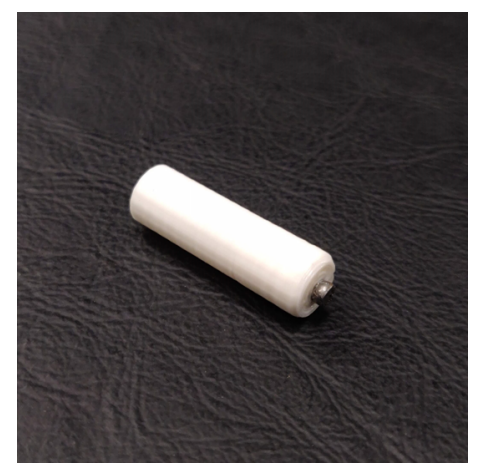

Figure 3.4: A tether bullet.

\subsubsection{Target Impact Surface}

The front panel of the target platform is modified to allow impacts to occur without causing damage to the normally black semi-transparent acrylic surface. A piece of painted sheet metal is mounted to the face of the panel at 8 points to increase durability. Magnets are attached to the panel at the predicted locations where the tether bullets will impact. This allows the magnetic tips of the bullets to stick to the panels and establish a tethered connection between the two platforms. The modified front panel of the platform and magnets are seen in Fig. 3.5.

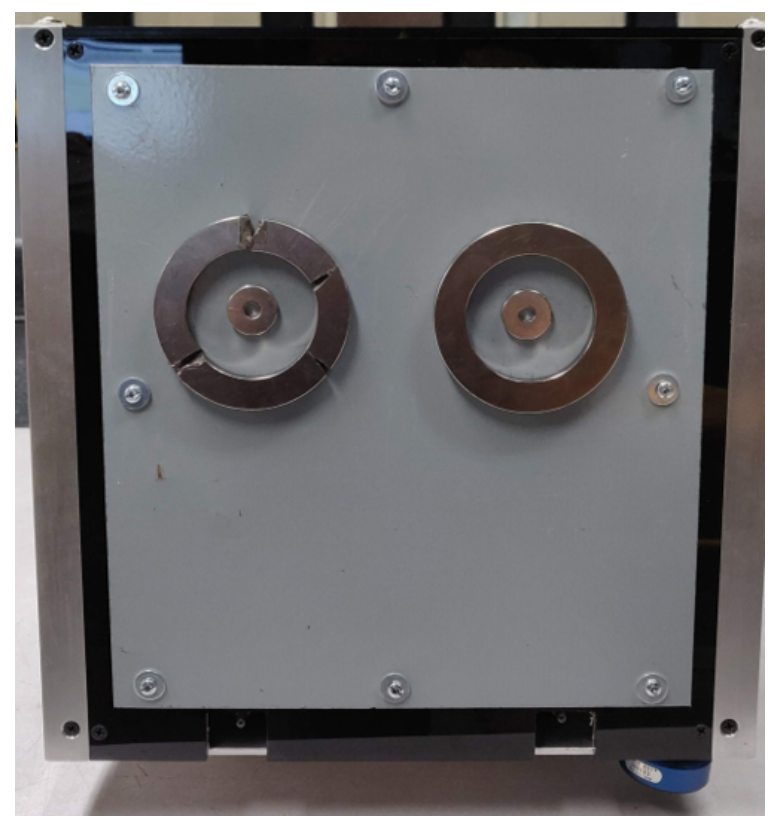

Figure 3.5: Target impact panel. 


\subsubsection{Tether}

Hovell [46] used a tether comprised of 90\% nylon, 10\% cotton, and a nonlinear stiffness to demonstrate the passive stabilization capabilities of the novel tether configuration. The tethers nonlinear elastic properties heightened its abilities to dampen the angular rates of a captured object. The same tether material was not used for validating the deployment motion of the novel tether. In order to effectively experimentally test the planar environment the deployment of the bullets and tether were maintained as horizontal to the surface as possible. Due to its mass, the nonlinear tether was not capable of being deployed over the required distance while maintaining a reasonable horizontal trajectory. The tether material used was Northern Sport's $11.3 \mathrm{~kg}$ "Northern Braid" braided polyethylene fishing line. It has no relevant elastic properties. 


\section{Chapter 4}

\section{Planar Tether Deployment and Complete Capture Scenario}

This chapter presents the planar dynamics of the Spacecraft Operations Testbed (SPOT) platforms within the SPOT, the planar tether deployment, and post-capture tethered spacecraft system (TSS). The same methods presented in Chap. 2 used to model and simulate the tether deployment and complete capture scenario are redefined within the planar environment outlined in Chap. 3.

\subsection{Introduction}

To validate the tether deployment and complete capture scenario the numerical models must be compare with experimental results. The planar environment of the SPOT is used to perform these experiments. As such, the numerical models presented in Chap. 2 must be redefined to be representative of the planar environment. Planar numerical models can be compared to experimental results to determine how accurate they are at predicting the motion of the tether during deployment, and the motion of the platforms during the complete capture scenario. This chapter presents the equations of motion for both SPOT platforms, the tether deployment, and post-capture phases in a planar environment. It builds on Hovell's [46] post-capture stabilization phase work by combining with the deployment phase to present the planar complete capture scenario.

\subsection{Planar Dynamics}

To create a planar representation of the 6 degrees of freedom (DOF) of movement of the on-orbit simulation, they must be reduced down to only 3 DOF. Translation is possible about two axes within a plane, and rotation is possible about a single axis perpendicular to the plane. The planar simulation consists of two SPOT platforms 
representing the target and chaser spacecraft. There are two phases of the simulation: the tether deployment, and post-capture stabilization phases. The contact phase is omitted as the impact force the tether bullets impart onto the target platform is negligible compared to the force required to move the platform. In order to create a relative angular rate between the two platforms, the actively controlled platform (the chaser) orbits around the uncooperative platform (the target) that is continually rotating in place. This scenario is chosen to create a differential in angular velocity between the two platforms, thereby emulating a non-ideal pose tracking maneuver.

The tether is initially attached to the face of the chaser platform. Both tether bullets are deployed perpendicular to the platform face. Figure 4.1 depicts the TSS configuration post-capture post-capture. The tether connected to the chaser is denoted as the main tether and the two tethers connected to the target platform are denoted as the sub-tethers. The point where the main tether splits into sub-tethers is denoted the junction.

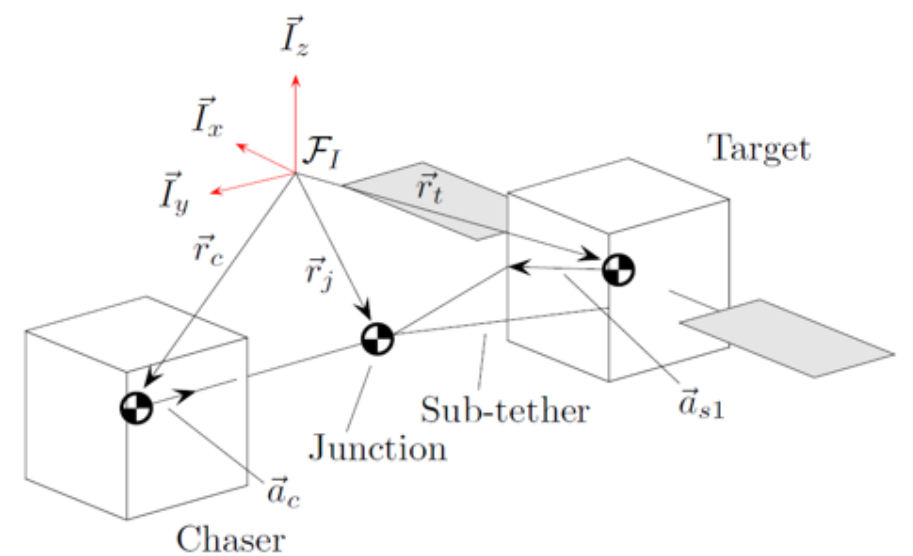

Figure 4.1: Reference frame and vector definition for planar dynamics modeling. For clarity, $\mathcal{F}_{c}, \mathcal{F}_{t}$, and $\vec{a}_{s_{2}}$ are omitted. [46].

During the deployment phase, the tethers are modeled using the lumped mass node with massless spring-damper elements, and ANCF models. In both cases torsion effects are ignored. In the post-capture stabilization phase the tethers are modeled with massless spring-damper elements connected at the junction node. All tether models ignore torsion. Additionally, the spring-damper models ignores bending effects. A net modeled at the end of the tethers is omitted as it is not compatible in the laboratory 
environment used to validate the numerical models.

This section presents the planar equations used to simulate the chaser and target spacecraft, and the tether during deployment and post-capture.

\subsubsection{Dynamics Formulation}

Figure 4.1 shows the configuration of the TSS. The inertial reference frame is given as $\mathcal{F}_{I}$, and the vector position vector of the center of mass of the target with respect to the origin of $\mathcal{F}_{I}$ is given as $\vec{r}_{t}$. The vector $\vec{r}_{t}$ has two-dimensional components, $\mathbf{r}_{t}$, in $\mathcal{F}_{I}$ such that:

$$
\vec{r}_{t}=\overrightarrow{\mathcal{F}}_{I}^{\mathrm{T}} \mathbf{r}_{t}
$$

Similarly, the two-dimensional positions of the center of mass of the chaser and a given tether lumped mass node of the tether are given as:

$$
\begin{aligned}
\vec{r}_{c} & =\overrightarrow{\mathcal{F}}_{I}^{\mathrm{T}} \mathbf{r}_{c} \\
\vec{r}_{n_{i}} & =\overrightarrow{\mathcal{F}}_{I}^{\mathrm{T}} \mathbf{r}_{n_{i}}
\end{aligned}
$$

where $\mathbf{r}_{c}$ and $\mathbf{r}_{n_{i}}$ are the two-dimensional components of the chaser and $i^{\text {th }}$ node position vectors in the inertially fixed reference frame, respectively.

From these vector and reference frame definitions, the $3 \mathrm{DOF}$, that is translation along $\vec{I}_{x}$ and $\vec{I}_{y}$ and rotation about $\vec{I}_{z}$, equations of motion for the system are presented.

\subsubsection{Rotational Motion}

Rotational motion is only applied to the two SPOT platforms. In a planar environment, rotational dynamics only consist of one DOF. To model this, Euler's equations are used to describe the platforms motion about a single axis the body-fixed reference frame $\mathcal{F}_{B}[47]$

$$
\mathrm{J} \dot{\omega}+\omega^{\times}(\mathrm{J} \omega)=\tau
$$


where $\boldsymbol{\omega}$ is the components of the angular rate vector in the body-fixed reference frame, $\mathcal{F}_{B}$, $\mathbf{J}$ is the inertia matrix of the target, and $\boldsymbol{\tau}$ is the sum of external torque applied to the target.

By assuming that $\mathcal{F}_{B}$ is aligned with the principal axes of the body, $\mathbf{J}$ can be simplified to:

$$
\mathbf{J}=\left[\begin{array}{ccc}
J_{x x} & 0 & 0 \\
0 & J_{y y} & 0 \\
0 & 0 & J_{z z}
\end{array}\right]
$$

where $J_{x x}, J_{y y}$, and $J_{z z}$ denote the principal moments of inertia. Due to the single axis of rotation of the planar environment, the angular rate vector components will always have the form:

$$
\boldsymbol{\omega}=\left[\begin{array}{l}
0 \\
0 \\
\omega
\end{array}\right]
$$

which eliminates the gyroscopic term in Eq. (4.4), simplifying it to:

$$
J_{z z} \dot{\omega}=\tau_{z}
$$

\subsubsection{Translational Motion}

In the planar environment there is $2 \mathrm{DOF}$ of translational motion. To model the target, chaser, and tether nodes linear motion, Newton's second law is used:

$$
\begin{aligned}
& \mathbf{F}=m \ddot{\mathbf{r}_{x}} \\
& \mathbf{F}=m \ddot{\mathbf{r}_{y}}
\end{aligned}
$$

where $\mathbf{F}$ is the net force vector components acting on a given body in $\mathcal{F}_{I}, m$ is the mass of the body in question, and $\ddot{\mathbf{r}}$ is the resulting inertial acceleration vector components in $\mathcal{F}_{I}$.

During deployment while the tether is not under tension, forces exerted on the 
chaser platform are due to the impulse generated during tether deployment, and no fores due to the tether are exerted on the target platform. Post-capture, the main tether and sub-tethers exert a force on each platform while in tension.

\subsubsection{Planar Deployment Dynamics Formulation - Lumped Mass Spring- Damper Model}

The planar massless spring-damper numerical model is nearly identical to the model presented in Chap. 2.2.4, with the exception that there is no translational motion in the $z$-axis. With this restriction, the tension force, $\mathbf{F}_{i k}$, between nodes $i$ and $k$ is expressed as:

$$
\mathbf{F}_{i k}=\left[\begin{array}{c}
F_{i k_{x}} \\
F_{i k_{y}}
\end{array}\right]= \begin{cases}F_{i k} \hat{\mathbf{r}}_{i k}, & \text { for } r_{i k}>L_{0} \\
0, & \text { otherwise }\end{cases}
$$

where

$$
\hat{\mathbf{r}}_{i k}=\frac{\mathbf{r}_{i k}}{\left\|\mathbf{r}_{i k}\right\|}, \quad \mathbf{r}_{i k}=\left[\begin{array}{c}
r_{i_{x}}-r_{k_{x}} \\
r_{i_{y}}-r_{k_{y}}
\end{array}\right]
$$

where $\mathbf{r}_{i k}$ is the relative position vector between the $i^{\text {th }}$ and $k^{\text {th }}$ nodes.

Aside from this restriction to achieve planar motion, the planar spring-damper model is identical to that which is presented in Chap. 2.2.4.

\subsubsection{Planar Deployment Dynamics Formulation - Absolute Nodal Coor- dinate Formulation}

The planar Absolute Nodal Coordinate Formulation (ANCF) dynamical model is identical to the 3 DOF model previously presented in Chap. 2.2.5. To account for planar motion, one degree of movement can be removed to create the planar environment by fixing the $z$-axis values of absolute nodal coordinates to be a constant 0 . This has the effects of restricting the motion of the tether to only the $x-y$ plane. The resulting absolute nodal coordinates of a tether element are expressed as:

$$
\mathbf{e}_{\mathbf{i}}=\left[e_{1}, e_{2}, 0, e_{4}, e_{5}, 0, e_{7}, e_{8}, 0, e_{10}, e_{11}, 0\right]
$$


Aside from this restriction to create planar motion, the planar ANCF model is identical to that which is presented in Chap. 2.2.5.

\subsubsection{Planar Post-Capture Dynamics Formulation - Spring-Damper Tether Model}

In order to numerically simulate Eqs. (4.7), (4.8), and (4.9) in the post-capture stabilization phase the forces and torques imparted on the chaser and the target must be quantified. Each tether in the post-capture phase is modeled as a massless springdamper system, as represented in Fig. 2.2 in Chap. 4.2.3. This is different than the deployment model, as the tether is not discretized into many nodes and tether element sections. The entire tether is represented by one single spring-damper element in the case of the single tether, and each of the main and two sub-tethers are represented by one single spring-damper element in the case of the sub-tether.

Hovell's [46] work done to model the post-capture stabilization process is presented below. The tether model has been modified with the omission of a nonlinear spring constant. This diversion from Hovell's [46] nonlinear model is due to the tether used for experimental validation having no nonlinear elastic properties. The nonlinear spring constant has been replaced with a constant spring constant.

In the post-capture detumbling phase the two sub-tethers are attached to the target as shown in Fig. 4.1. The attachment point vector components with respect to the target's center of mass are:

$$
\begin{aligned}
\operatorname{att}_{s_{1}} & \equiv\left[\begin{array}{l}
a t t_{s_{1}, x} \\
a t t_{s_{1}, y}
\end{array}\right]=\overrightarrow{\mathcal{F}}_{t} \cdot a \vec{t} t_{s_{1}} \\
\operatorname{att}_{s_{2}} & \equiv\left[\begin{array}{l}
a t t_{s_{2}, x} \\
a t t_{s_{2}, y}
\end{array}\right]=\overrightarrow{\mathcal{F}}_{t} \cdot a \vec{t} t_{s_{2}}
\end{aligned}
$$

and the attachment point of the main tether to the chaser is given as:

$$
\operatorname{att}_{c} \equiv\left[\begin{array}{l}
a t t_{c, x} \\
a t t_{c, y}
\end{array}\right]=\overrightarrow{\mathcal{F}}_{c} \cdot a \overrightarrow{t t} t_{c}
$$


The main tether vector, $\vec{L}_{m}$, attaches the chaser to the junction. The two subtether vectors, $\vec{L}_{s_{1}}$ and $\vec{L}_{s_{2}}$, extend from the junction to the two respective attachment points on the target. The tether vector coordinates in $\mathcal{F}_{I}$ are given by:

$$
\begin{aligned}
& \mathbf{L}_{m}=\mathbf{r}_{j}-\mathbf{r}_{c}-\mathbf{C}_{\mathrm{BI}}\left(\theta_{c}\right) \text { att }_{c} \\
& \mathbf{L}_{s_{1}}=\mathbf{r}_{t}+\mathbf{C}_{\mathrm{BI}}\left(\theta_{t}\right) \mathbf{a t t}_{s_{1}}-\mathbf{r}_{j} \\
& \mathbf{L}_{s_{2}}=\mathbf{r}_{t}+\mathbf{C}_{\mathrm{BI}}\left(\theta_{t}\right) \operatorname{att}_{s_{2}}-\mathbf{r}_{j}
\end{aligned}
$$

The stretches in the main, and two sub-tethers are:

$$
\begin{aligned}
& \Delta L_{m}=\left\|\mathbf{L}_{m}\right\|-L_{m, 0} \\
& \Delta L_{s_{1}}=\left\|\mathbf{L}_{s_{1}}\right\|-L_{s_{1}, 0} \\
& \Delta L_{s_{2}}=\left\|\mathbf{L}_{s_{2}}\right\|-L_{s_{2}, 0}
\end{aligned}
$$

where $L_{m, 0}, L_{s_{1}, 0}$, and $L_{s_{2}, 0}$ are the unstretched lengths of the main tether and subtethers, respectively.

The angle $\theta_{t}$ represents the rotation about $\vec{I}_{z}$ to go from $\mathcal{F}_{I}$ to $\mathcal{F}_{t}$. Similarly, $\theta_{c}$ describes how $\mathcal{F}_{I}$ is rotated about $\vec{I}_{z}$ to obtain $\mathcal{F}_{c}$. The two-dimensional attitude matrix $\mathbf{C}_{\mathrm{BI}}(\theta)$ is a pure rotation of $\theta$ about the $\vec{I}_{z}$ axis which rotates vector components from a body frame to $\mathcal{F}_{I}$, given by

$$
\mathbf{C}_{\mathrm{BI}}(\theta)=\left[\begin{array}{rr}
\cos (\theta) & -\sin (\theta) \\
\sin (\theta) & \cos (\theta)
\end{array}\right]
$$

such that vector components are rotated from either body frame to $\mathcal{F}_{I}$, as follows:

$$
\mathbf{x}_{I}=\mathbf{C}_{\mathrm{BI}}^{T}(\theta) \mathbf{x}_{B}
$$

where $\mathbf{x}_{B}$ are the vector coordinates in a body frame and $\mathbf{x}_{I}$ are the vector components in $\mathcal{F}_{I}$.

Using this, the resulting forces in the main tether and sub-tethers are described as: 


$$
\begin{aligned}
& \mathbf{F}_{m}=\left\{\begin{array}{lr}
{\left[k \Delta L_{m}+c\left(\mathbf{v}_{j}-\mathbf{v}_{c}-\mathbf{C}_{\mathrm{BI}}\left(\theta_{c}\right)\left[\begin{array}{r}
-\omega_{c} a t t_{c, y} \\
\omega_{c} a t t_{c, x}
\end{array}\right]\right)^{\mathrm{T}} \frac{\mathbf{L}_{m}}{\left\|\mathbf{L}_{m}\right\|}\right]} & \frac{\mathbf{L}_{m}}{\left\|\mathbf{L}_{m}\right\|}, \\
0, & \text { for } \Delta L_{m}>0
\end{array}\right. \\
& \mathbf{F}_{s_{1}}=\left\{\begin{array}{lr}
{\left[k \Delta L_{s_{1}}+c\left(\mathbf{v}_{t}+\mathbf{C}_{\mathrm{BI}}\left(\theta_{t}\right)\left[\begin{array}{c}
-\omega_{t} a t t_{s_{1}, y} \\
\omega_{t} a t t_{s_{1}, x}
\end{array}\right]-\mathbf{v}_{j}\right)^{\mathrm{T}} \frac{\mathbf{L}_{s_{1}}\left\|\mathbf{L}_{s_{1}}\right\|}{\left\|\mathbf{L}_{s_{1}}\right\|},\right.} \\
0 & \text { for } \Delta L_{s_{1}}>0 \\
\mathbf{0} & \text { otherwise }
\end{array}\right. \\
& \mathbf{F}_{s_{2}}=\left\{\begin{array}{lr}
{\left[k \Delta L_{s_{2}}+c\left(\mathbf{v}_{t}+\mathbf{C}_{\mathrm{BI}}\left(\theta_{t}\right)\left[\begin{array}{c}
-\omega_{t} a t t_{s_{2}, y} \\
\omega_{t} a t t_{s_{2}, x}
\end{array}\right]-\mathbf{v}_{j}\right)^{\mathrm{T}} \frac{\mathbf{L}_{s_{2}}}{\left\|\mathbf{L}_{s_{2}}\right\|}\right]} & \frac{\mathbf{L}_{s_{2}}}{\left\|\mathbf{L}_{s_{2}}\right\|}, \\
0, & \text { for } \Delta L_{s_{2}}>0
\end{array}\right.
\end{aligned}
$$

where $\mathbf{v}_{j}$ is the velocity components of the junction in $\mathcal{F}_{I}$. The resulting forces, $\mathbf{F}_{m}, \mathbf{F}_{s_{1}}$, and $\mathbf{F}_{s_{2}}$ are calculated in $\mathcal{F}_{I}$, and are only non-zero when the tethers are stretched. The torque delivered to each spacecraft is expressed in its body frame. Therefore, as with the single tether configuration, the tether forces must be rotated into the appropriate body-fixed reference frame, through:

$$
\begin{array}{r}
\mathbf{F}_{m}^{c} \equiv\left[\begin{array}{c}
F_{m, x}^{c} \\
F_{m, y}^{c}
\end{array}\right]=\mathbf{C}_{\mathrm{BI}}\left(\theta_{c}\right)^{\mathrm{T}} \mathbf{F}_{m} \\
\mathbf{F}_{s_{1}}^{t} \equiv\left[\begin{array}{c}
F_{s_{1}, x}^{t} \\
F_{s_{1}, y}^{t}
\end{array}\right]=\mathbf{C}_{\mathrm{BI}}\left(\theta_{t}\right)^{\mathrm{T}} \mathbf{F}_{s_{1}} \\
\mathbf{F}_{s_{2}}^{t} \equiv\left[\begin{array}{c}
F_{s_{2}, x}^{t} \\
F_{s_{2}, y}^{t}
\end{array}\right]=\mathbf{C}_{\mathrm{BI}}\left(\theta_{t}\right)^{\mathrm{T}} \mathbf{F}_{s_{2}}
\end{array}
$$


where, $\mathbf{F}_{m}^{c}$ is the main tether force vector components in $\mathcal{F}_{c}$, and $\mathbf{F}_{s_{1}}^{t}$ and $\mathbf{F}_{s_{2}}^{t}$ are the force vector components of the two sub-tethers in $\mathcal{F}_{t}$.

\subsubsection{Planar Equations of Motion}

The equations of motion of the chaser, target, and tether can be broken down into two phases: deployment of the tether, and establishment of the tethered spacecraft system (TSS) for the post-capture stabilization of the target.

To model the motion of the platforms and tethers, all forces contributing to translational motion are included in Eq. (4.8) and (4.9) for each body. Rotational motion caused by torque effects, as described by Eq. (4.7), are only modeled for the platforms as the tether nodes are considered point masses.

During the tether deployment phase the resulting net force on the target in $\mathcal{F}_{I}$ is only due to the platform thrusters that are used to translate or rotate it. It is given by:

$$
\left[\begin{array}{c}
\ddot{r}_{t, x} \\
\ddot{r}_{t, y} \\
\dot{\omega}_{t}
\end{array}\right]=\left[\begin{array}{c}
\frac{F_{\text {control }, t, x}}{m_{t}} \\
\frac{F_{\text {control }, t, y}}{m_{t}} \\
\frac{\tau_{c o n t r o l, t}}{J_{z z, t}}
\end{array}\right]
$$

The chaser platform is similar, with the inclusion of a one-time impulse force, $\mathbf{F}_{\text {impulse }}$, that occurs when the tether is deployed. Due to there being two bullets that are deployed, this force is symmetrical and does not impart a torque on the platform.

$$
\left[\begin{array}{c}
\ddot{r}_{c, x} \\
\ddot{r}_{c, y} \\
\dot{\omega}_{c}
\end{array}\right]=\left[\begin{array}{c}
\frac{F_{\text {control }, c, x}-F_{\text {impulse }, x}}{m_{c}} \\
\frac{F_{\text {control }, c, y}-F_{\text {impulse }, y}}{m_{c}} \\
\frac{\tau_{c o n t r o l, c}}{J_{z z, c}}
\end{array}\right]
$$

where:

$$
\mathbf{F}_{\text {control }} \equiv\left[\begin{array}{c}
F_{\text {control }, x} \\
F_{\text {control }, y}
\end{array}\right], \quad \mathbf{F}_{\text {impulse }} \equiv\left[\begin{array}{c}
F_{\text {impulse }, x} \\
F_{\text {impulse }, y}
\end{array}\right]
$$

A given tether node $i$ experiences similar forces to the chaser before they are deployed from its surface as a result of being pulled by the proceeding node in the tether. A bullet will experience an additional initial impulse force. These forces are 
given by the conditions:

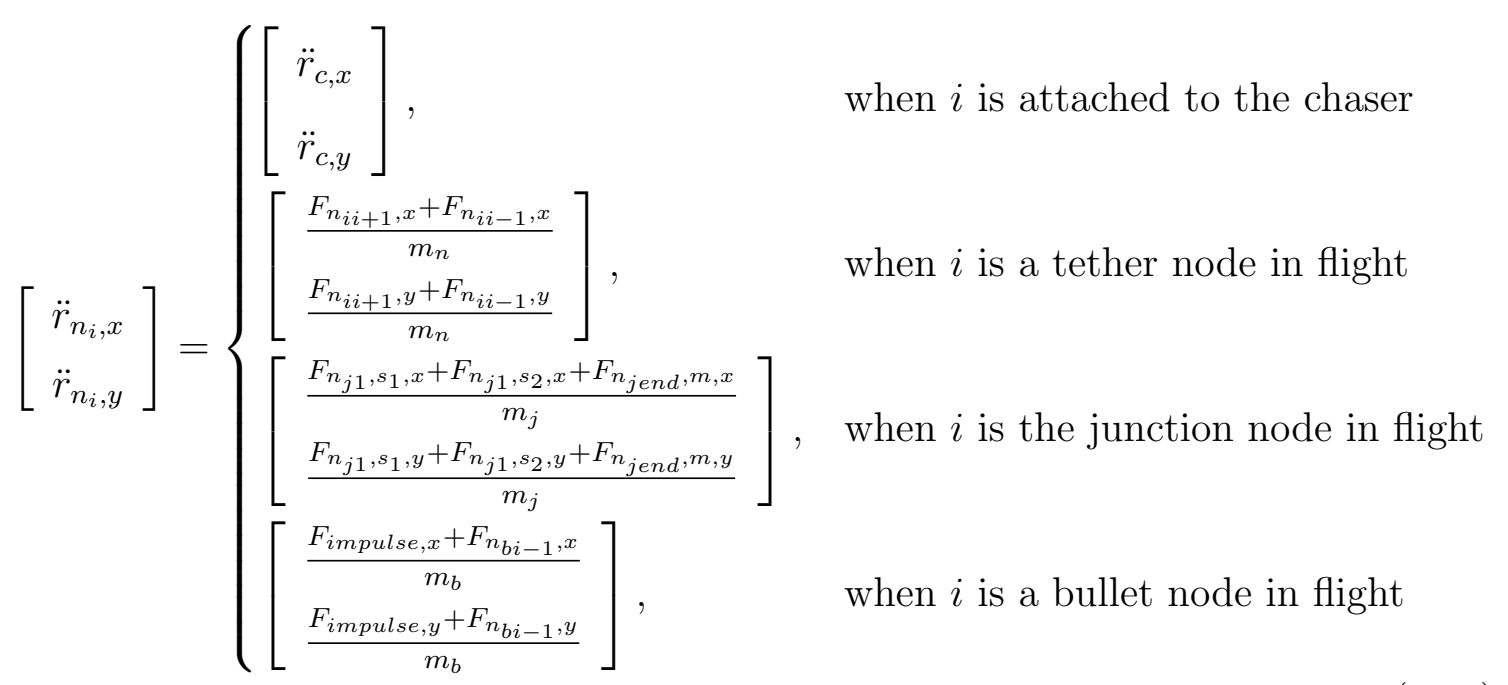

where the subscripts ' ' $s$ denotes a sub-tether, ' $m$ ' denotes the main tether, ' $j$ ' denotes the junction node, and ' ${ }_{i-1}$ ' and ' ${ }_{i+1}$ ' denote the node before and after node $i$ of the tether, respectively.

in question.

During the contact phase the tether bullet and target post-contact velocities are calculated and applied at the first step of the post-capture phase. Practically, the tether bullets are much smaller in mass than the target and the impulse imparted is negligible. Omitting this step in simulation has no consequential effects for the simulation. However, if a net at the end of the sub-tethers were to be included this step would be necessary.

During the post-capture stabilization phase the sub-tethers attach to the target platform while the main tether is attached to the chaser platform. The equations of motion are for the target are:

$$
\left[\begin{array}{c}
\ddot{r}_{t, x} \\
\ddot{r}_{t, y} \\
\dot{\omega}_{t}
\end{array}\right]=\left[\begin{array}{c}
\frac{-\left(F_{s_{1}, x}+F_{s_{2}, x}\right)}{m_{t}} \\
\frac{-\left(F_{s_{1}, y}+F_{s_{2}, y}\right)}{m_{t}} \\
\frac{\tau_{t, s_{1}}+\tau_{t, s 2}}{J_{z z, t}}
\end{array}\right]
$$


for the junction:

$$
\left[\begin{array}{c}
\ddot{r}_{j, x} \\
\ddot{r}_{j, y}
\end{array}\right]=\left[\begin{array}{c}
\frac{F_{s_{1}, x}+F_{s_{2}, x}-F_{m, x}}{m_{j}} \\
\frac{F_{s_{1}, y}+F_{s_{2}, y}-F_{m, y}}{m_{j}}
\end{array}\right]
$$

and for the chaser:

$$
\left[\begin{array}{c}
\ddot{r}_{c, x} \\
\ddot{r}_{c, y} \\
\dot{\omega}_{c}
\end{array}\right]=\left[\begin{array}{c}
\frac{F_{m, x}+F_{\text {control }, x}}{m_{c}} \\
\frac{F_{m, y}+F_{c o n t r o l, y}}{m_{c}} \\
\frac{\tau_{c, m}+\tau_{c o n t r o l}}{J_{z z, c}}
\end{array}\right]
$$

where:

$$
\mathbf{F}_{m} \equiv\left[\begin{array}{c}
F_{m, x} \\
F_{m, y}
\end{array}\right], \quad \mathbf{F}_{s_{1}} \equiv\left[\begin{array}{c}
F_{s_{1}, x} \\
F_{s_{1}, y}
\end{array}\right], \quad \mathbf{F}_{s 2} \equiv\left[\begin{array}{c}
F_{s_{2}, x} \\
F_{s_{2}, y}
\end{array}\right]
$$

where $\mathbf{F}_{m}$ represents the force vector components of the main tether in $\mathcal{F}_{I}, \mathbf{F}_{s_{1}}$ and $\mathbf{F}_{s_{2}}$ represent the force vector components of the two sub-tethers in $\mathcal{F}_{I}, \tau_{t, s_{1}}$ and $\tau_{t, s_{2}}$ denote the torques imparted to the target due to the two sub-tethers in $\mathcal{F}_{t}$, and $\tau_{c, m}$ denotes the torque applied to the chaser from the main tether segment in $\mathcal{F}_{c}$.

The resulting torques on the spacecraft are calculated as follows:

$$
\begin{aligned}
& \tau_{t, s_{1}}=a t t_{s_{1}, y} F_{s_{1}, x}^{t}-a t t_{s_{1}, x} F_{s_{1}, y}^{t} \\
& \tau_{t, s 2}=a t t_{s_{2}, y} F_{s_{2}, x}^{t}-a t t_{s_{2}, x} F_{s_{2}, y}^{t} \\
& \tau_{c, m}=a t t_{c, x} F_{m, y}^{c}-a t t_{c, y} F_{m, x}^{c}
\end{aligned}
$$

and are used with Eqs. (4.24) to (4.26) in Eqs. (4.34) to (4.36) to simulate the motion.

\subsection{Planar Numerical Simulations}

\subsection{Planar Tether Deployment Simulation}

The equations presented in this chapter are used to simulate the experimental environment of the SPOT presented in Chap. 3, the tether deployment experiment, and the planar complete capture scenario. The two SPOT platforms are represented in simulation by the red and black squares, with the line extending from the center to an edge on each denoting the front facing side of the platform. Tether elements are represented by gray lines while tether nodes are represented by black dots. In 
the spring-damper model tether nodes are evenly spaced along the front face of the platform, spanning from the center point between both tether bullets where the remaining tether is stored at a single point. In the ANCF model, the bullet nodes are places at the same positions as the spring-damper model, with the junction and platform connecting nodes at the midpoint between the two bullets. A planar tether deployment simulation is run for each deployment model. The parameters of these simulations are found in Table 4.1.

Table 4.1: Initial conditions and parameters for the planar tether deployment and post-capture scenario.

\begin{tabular}{cccccc}
\hline \hline Parameter & Value & Parameter & Value & Parameter & Value \\
\hline$A, \mathrm{~m}^{2}$ & $3.14 \times 10^{-6}$ & $m_{b}, \mathrm{~kg}$ & 0.05 & $L_{\text {main }}, \mathrm{m}$ & 0.28 \\
$E, \mathrm{~Pa}$ & $34.73 \times 10^{6}$ & $m_{n}, \mathrm{~kg}$ & 0.01 & $L_{\text {sub }}, \mathrm{m}$ & 0.28 \\
$I, \mathrm{~m}^{4}$ & $2.61 \times 10^{-6}$ & $m_{j}, \mathrm{~kg}$ & 0.015 & $k, \frac{\mathrm{N}}{\mathrm{m}}$ & 175 \\
$\rho, \mathrm{kg} / \mathrm{m}^{3}$ & 800 & $v_{b}, \frac{\mathrm{m}}{\mathrm{s}}$ & 0.75 & $c, \frac{\mathrm{Ns}}{\mathrm{m}}$ & 1.1 \\
$\beta$ & 0.3025 & $\gamma$ & 0.6 & $\#$ nodes $/$ tether & 5 \\
\hline \hline
\end{tabular}

Figures $4.2(\mathrm{a})$ to $4.2(\mathrm{k})$ depict the tether deployment using the spring-damper model in 0.1 second increments. Figures $4.3(\mathrm{a})$ to $4.3(\mathrm{k})$ depict the tether deployment using the ANCF model in 0.1 second increments. The $y$-axis positions of both platforms have been adjusted in Figs. 4.2 and 4.3 to better view the simulated SPOT platforms during the tether deployment. The axes shown and platform positions are not the true values of the simulation. This change is visual only and does not affect the results.

Figures 4.2(1) and 4.3(1) show the immediate TSS configuration post-capture of both models. Upon contacting the target, the tether dynamics are immediately switched over from the deployment tether model to the nonlinear spring damper outlined in Chap. 4.2.6. When the dynamics are switched, the contact positions with the chaser and the position of the junction are maintained.

In simulation, the novel tether configuration was capable of deploying nominally and contacting a target with a different angular velocity.

To validate the planar simulation and accompanying dynamical models, the tether models are compared against experimental results preformed in a planar environment 
in Chap. 5.2.
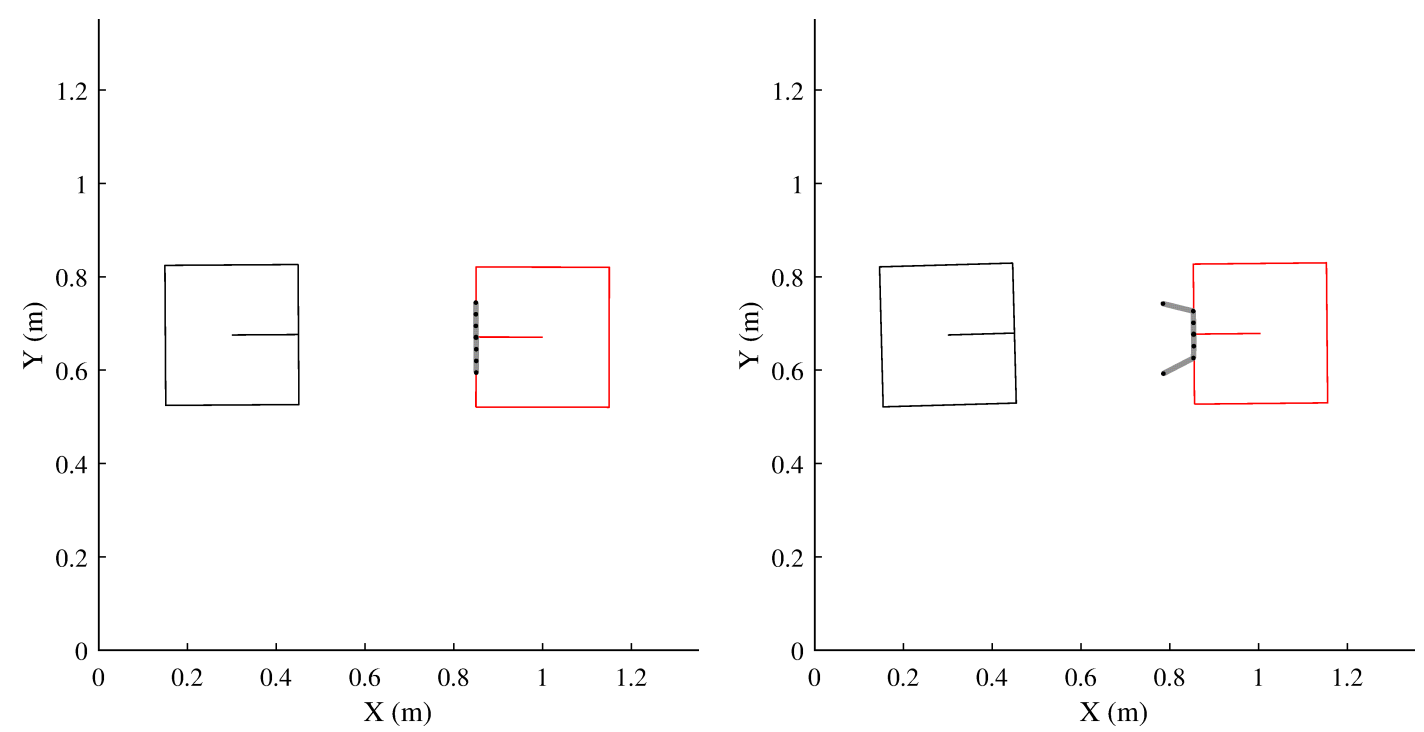

(a) Planar spring-damper model tether deploy- (b) Planar spring-damper model tether deployment initial configuration ment at $0.1 \mathrm{~s}$.
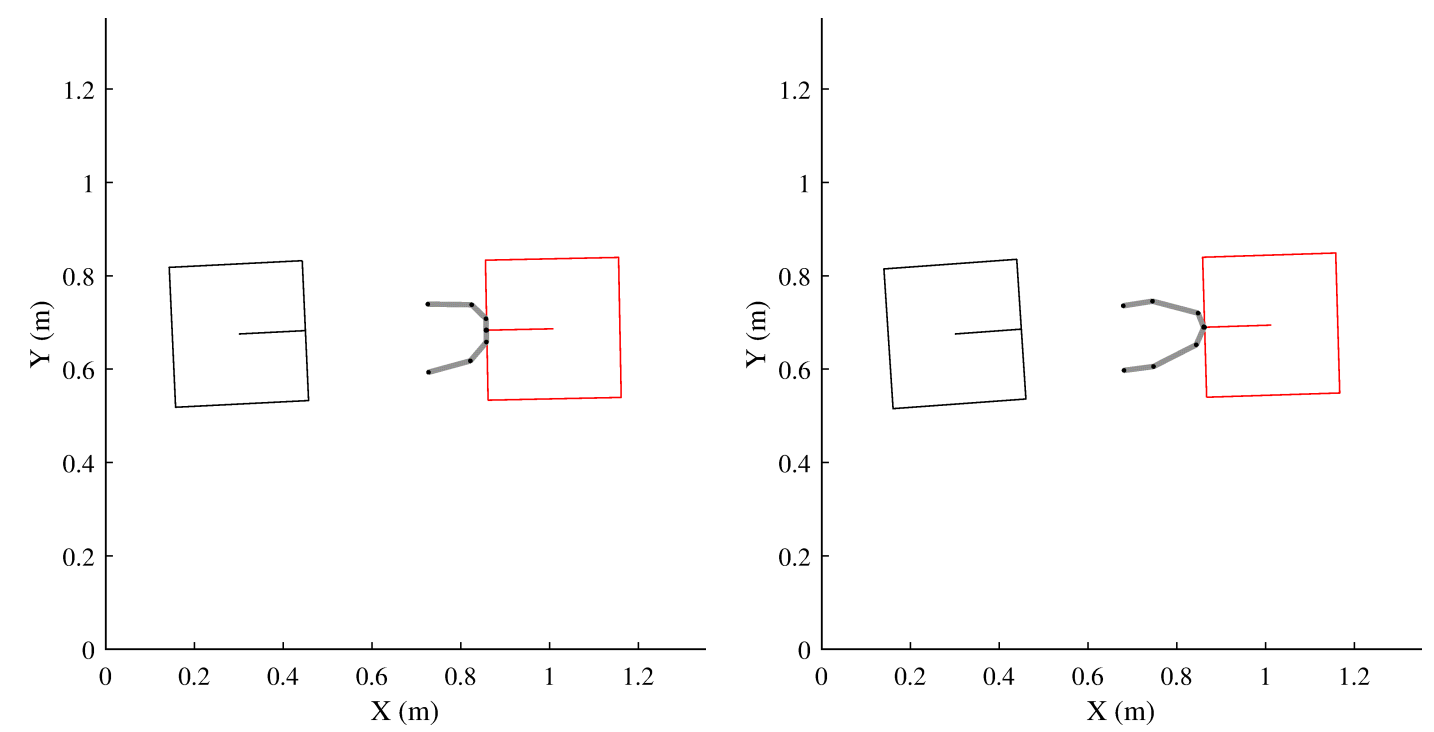

(c) Planar spring-damper model tether deploy- (d) Planar spring-damper model tether deployment at $0.2 \mathrm{~s}$. ment at $0.3 \mathrm{~s}$.

\subsection{Planar Complete Capture Scenario Simulation}

The complete capture scenario was preformed in simulation. The post-capture stabilization phase persisted for 60 seconds once the TSS had been established. Once 

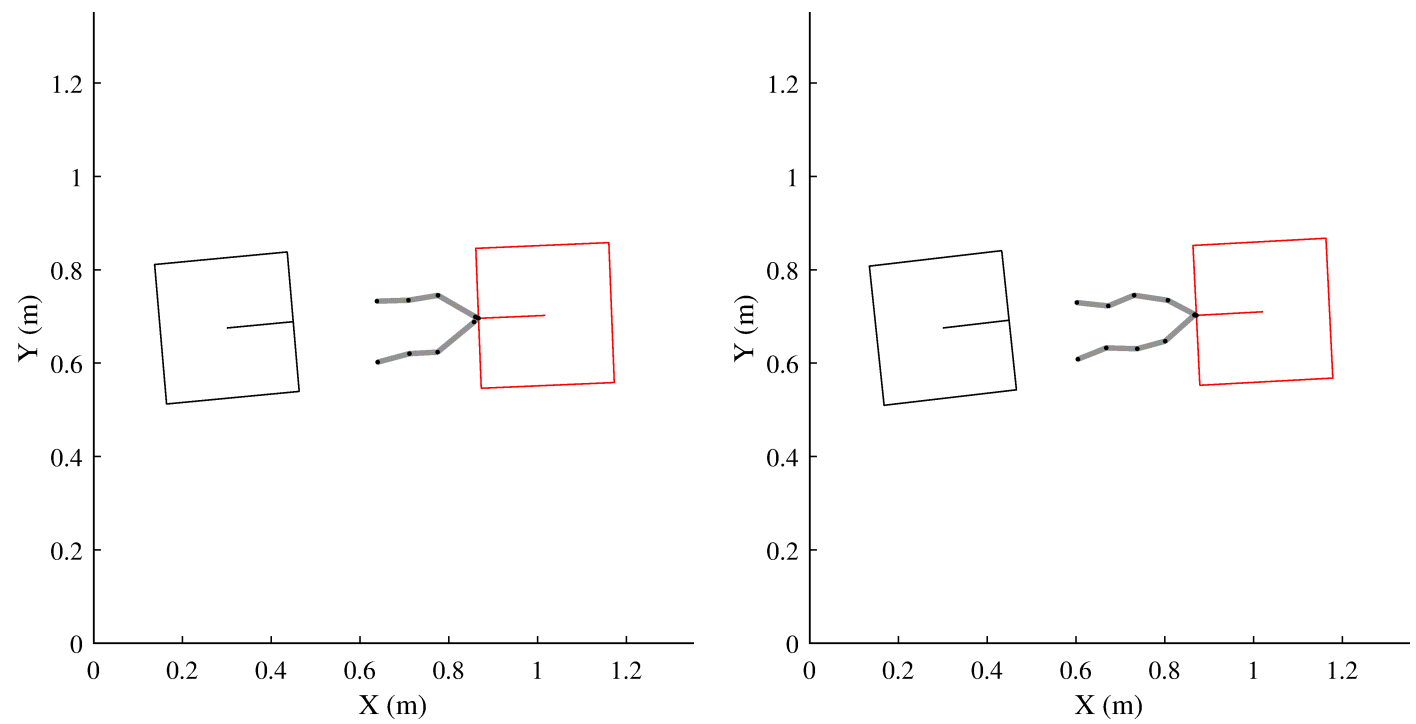

(e) Planar spring-damper model tether deploy- (f) Planar spring-damper model tether deployment at $0.4 \mathrm{~s}$. ment at $0.5 \mathrm{~s}$.
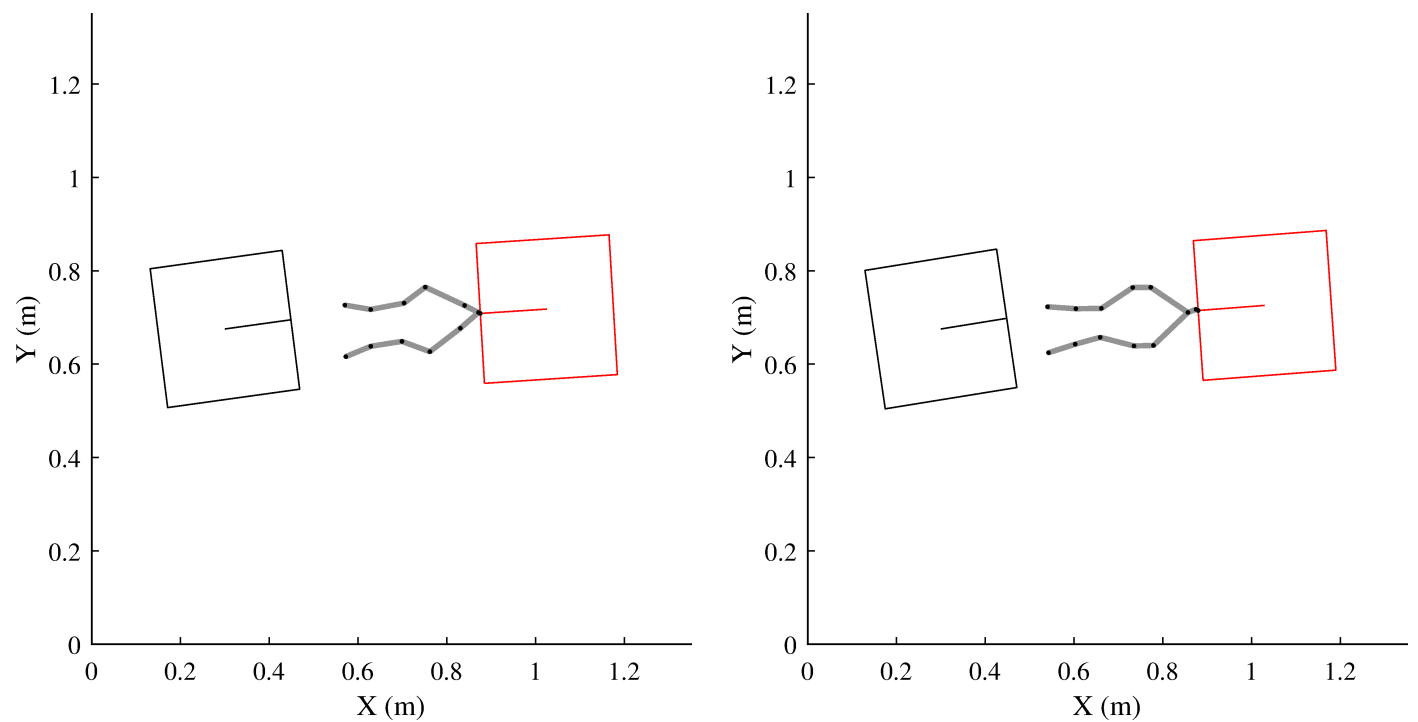

(g) Planar spring-damper model tether deploy- (h) Planar spring-damper model tether deployment at $0.6 \mathrm{~s}$. ment at $0.7 \mathrm{~s}$. 

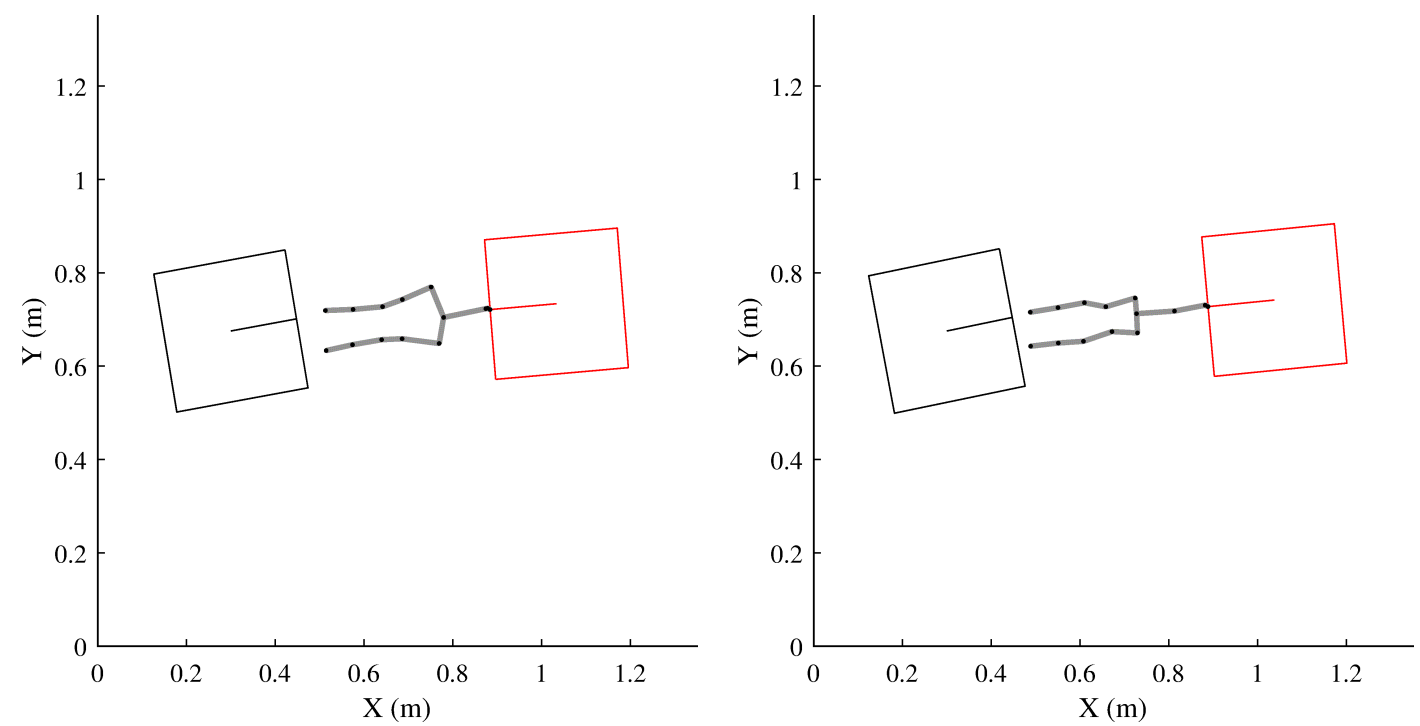

(i) Planar spring-damper model tether deploy- (j) Planar spring-damper model tether deployment at $0.8 \mathrm{~s}$. ment at $0.9 \mathrm{~s}$.
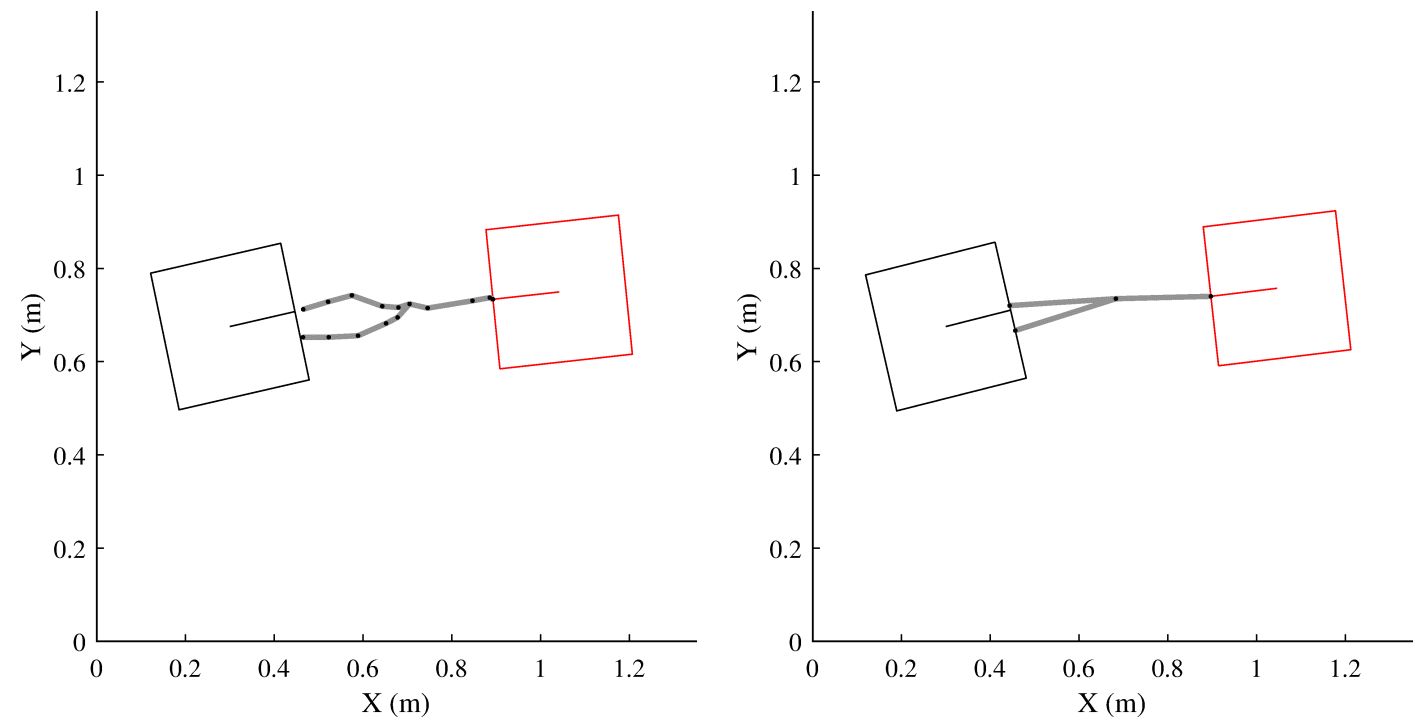

(k) Planar spring-damper model tether deploy- (l) Initial position of the planar spring-damper ment at $1.0 \mathrm{~s}$. model TSS post-capture at $1.1 \mathrm{~s}$.

Figure 4.2: Novel tether configuration deployment in a planar environment using the massless spring-damper model. 

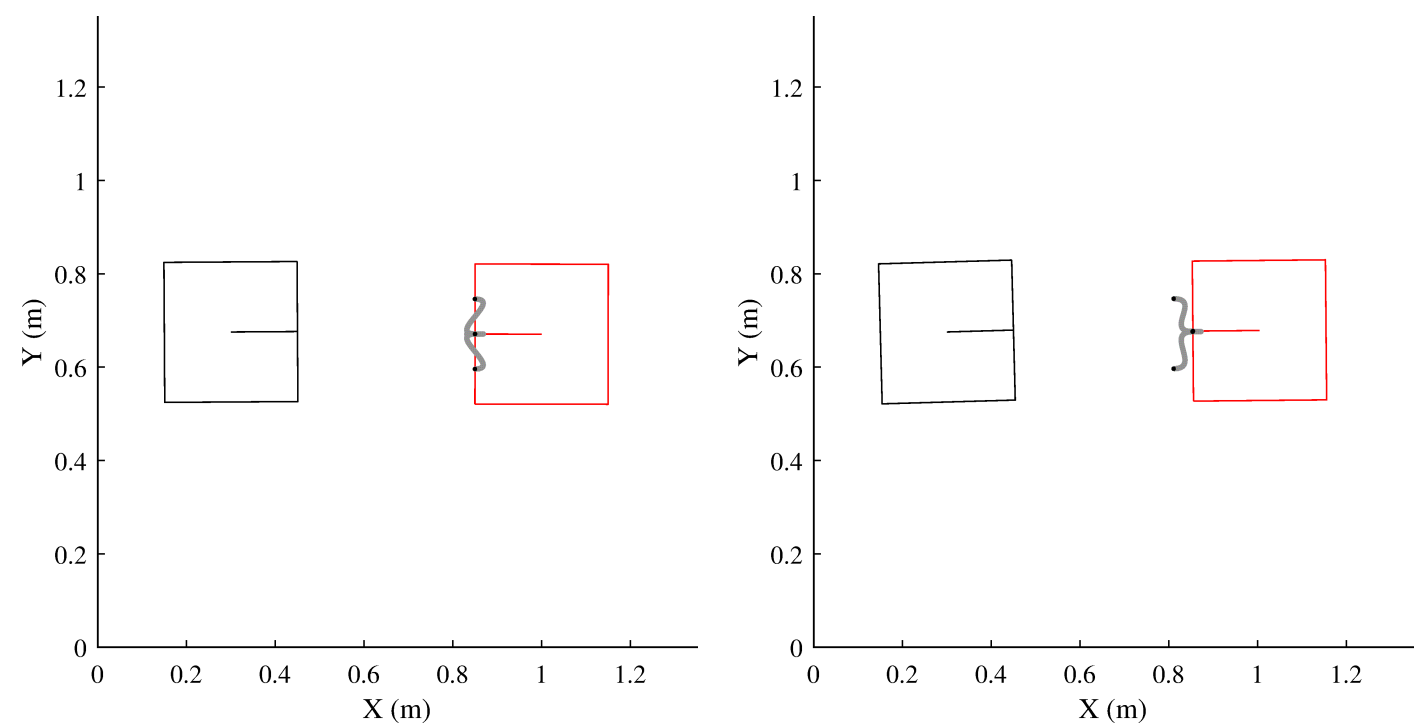

(a) Planar ANCF model tether deployment ini- (b) Planar ANCF model tether deployment at tial configuration $0.1 \mathrm{~s}$.
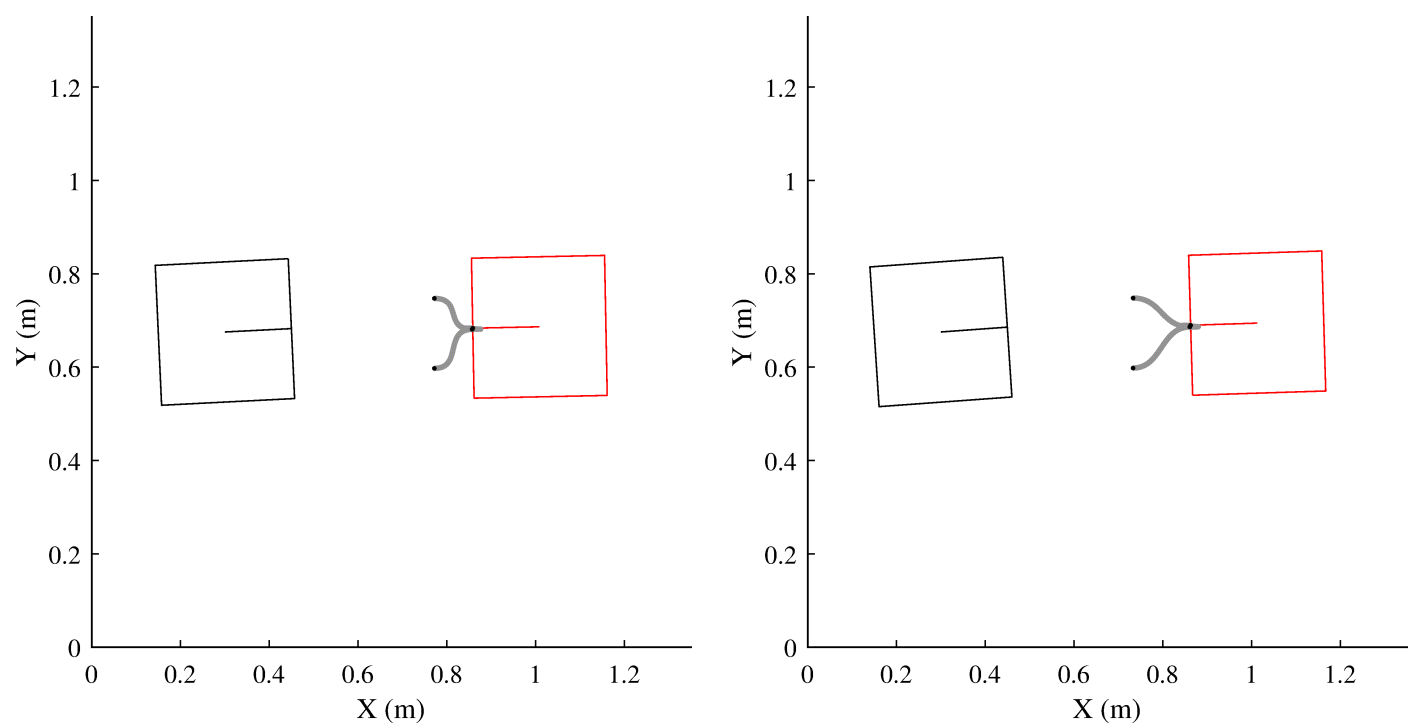

(c) Planar ANCF model tether deployment at (d) Planar ANCF model tether deployment at $0.2 \mathrm{~s}$. $0.3 \mathrm{~s}$. 

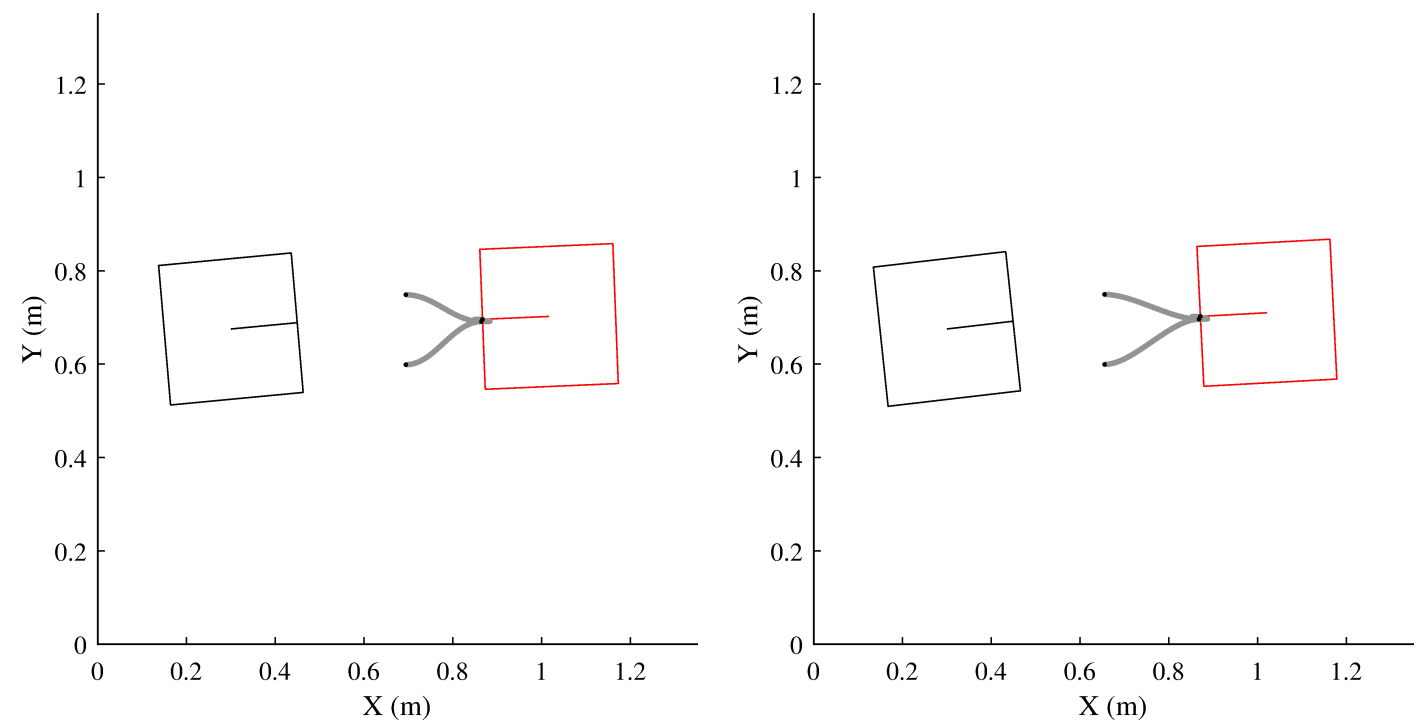

(e) Planar ANCF model tether deployment at (f) Planar ANCF model tether deployment at $0.4 \mathrm{~s}$. $0.5 \mathrm{~s}$.
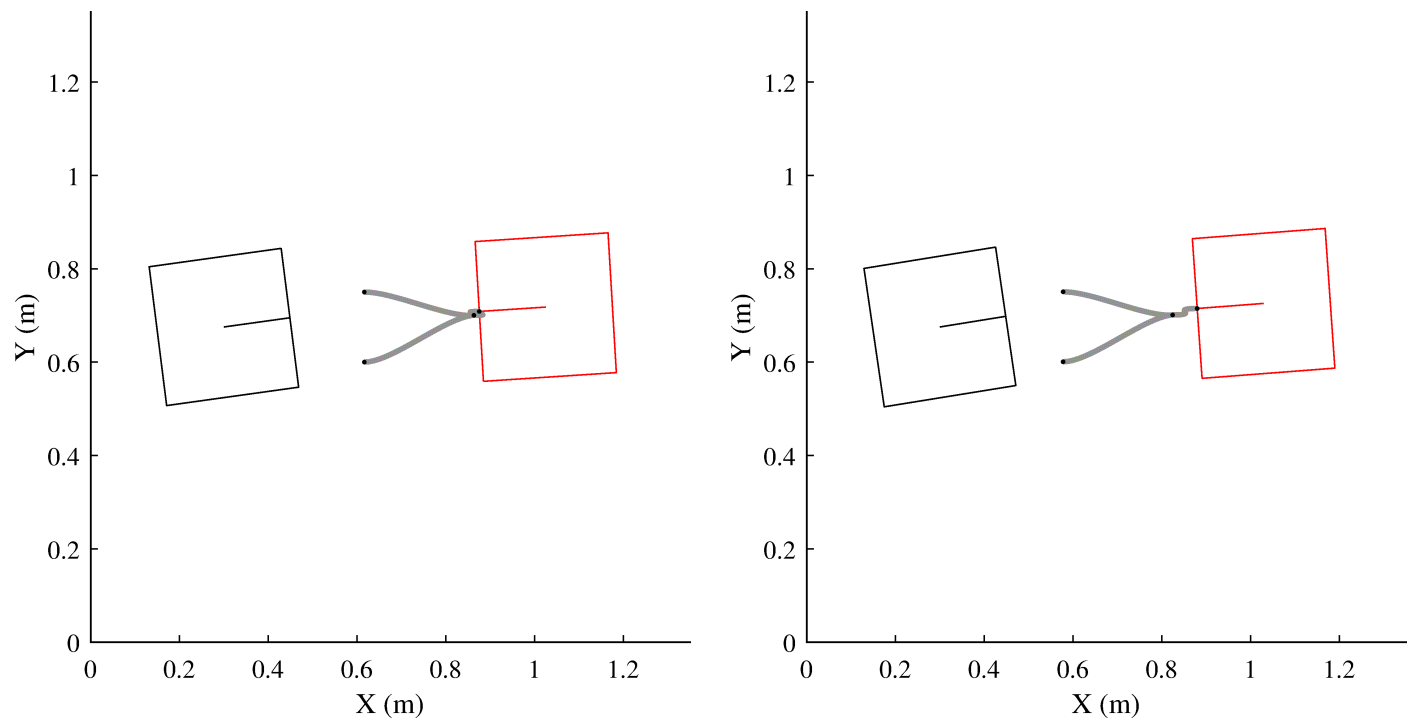

(g) Planar ANCF model tether deployment at (h) Planar ANCF model tether deployment at $0.6 \mathrm{~s}$. $0.7 \mathrm{~s}$. 

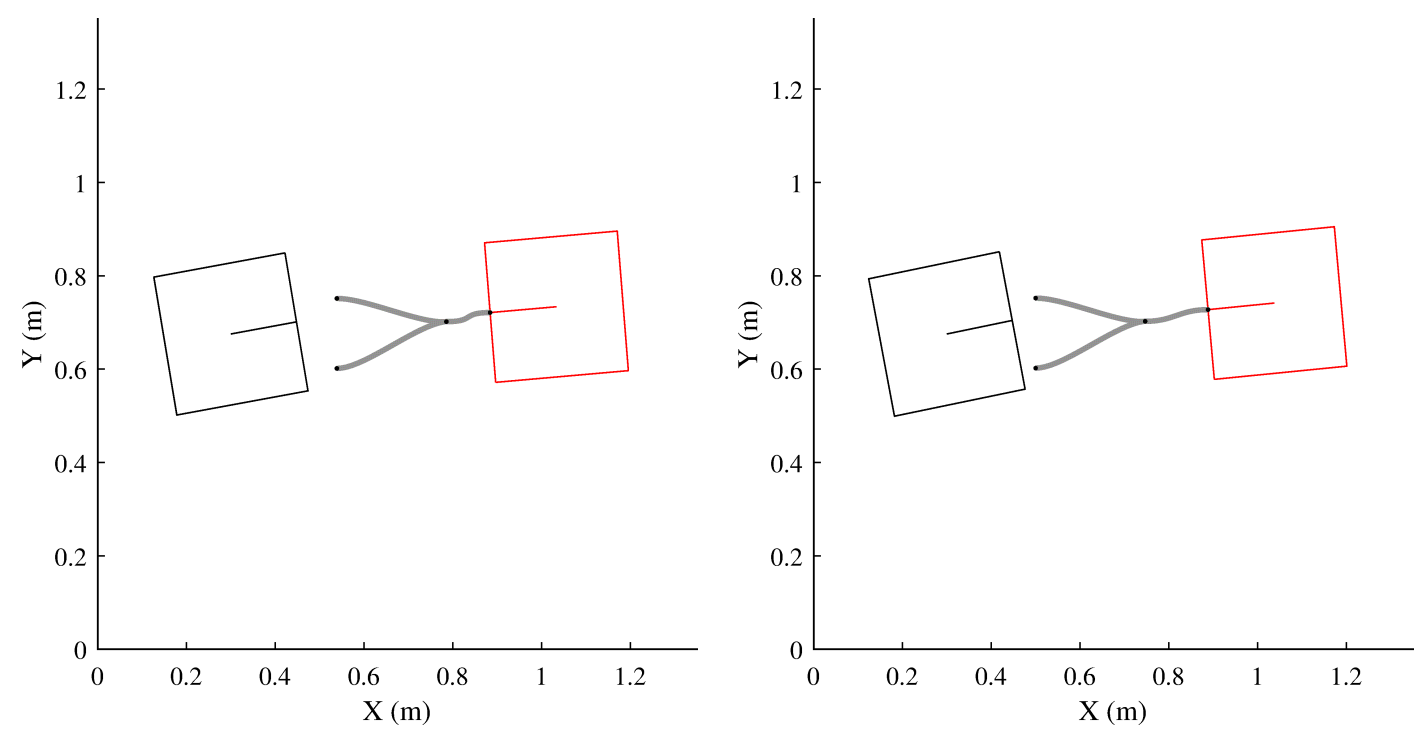

(i) Planar ANCF model tether deployment at (j) Planar ANCF model tether deployment at $0.8 \mathrm{~s}$. $0.9 \mathrm{~s}$.
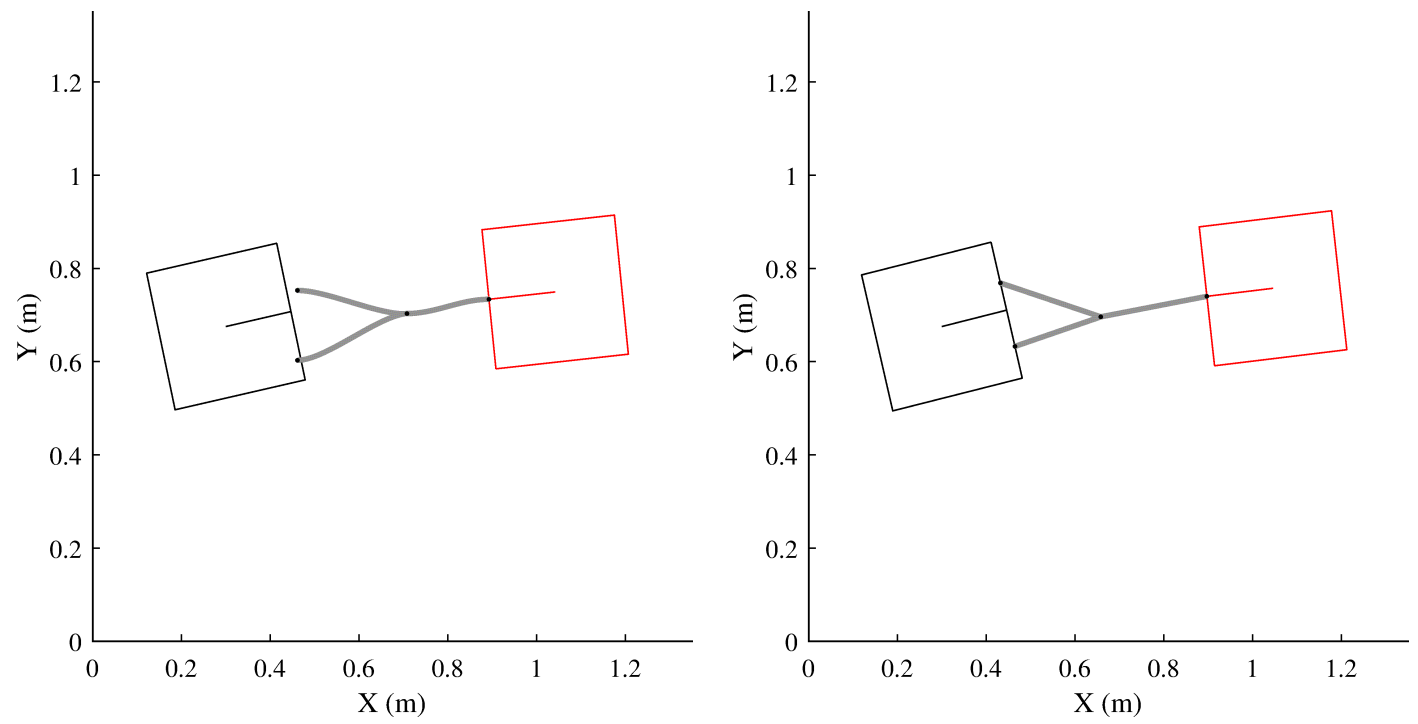

(k) Planar ANCF model tether deployment at (l) Initial position of the planar ANCF model $1.0 \mathrm{~s}$.

TSS post-capture at $1.1 \mathrm{~s}$.

Figure 4.3: Novel tether configuration deployment in a planar environment using the ANCF model. 
the tether was deployed both platforms ceased all active transnational motion and the chaser platform only used its thrusters to point in the direction of the target platform. Simulation parameters were unchanged from those in Table 4.1. The same tether connection points as shown in Figs. 4.2(l) and 4.3(l) were used.

Figures 4.4(a) and 4.4(c) show the positions of the chaser and target platforms over time. Figures 4.4(b) and 4.4(d) show the pointing angles of the platforms over time. All plots in Fig. 4.4 begin just before tether deployment occurs. This is because the motion before tether deployment is controlled and constant.

It is demonstrated that the complete capture scenario is possible in a planar environment. Videos of the planar tether deployment can be accessed at https: //tinyurl.com/yy9faazm.

To validate the planar complete capture scenario, the platforms positions and angles are compared with the experimental complete capture scenario in Chap. 5.3. 

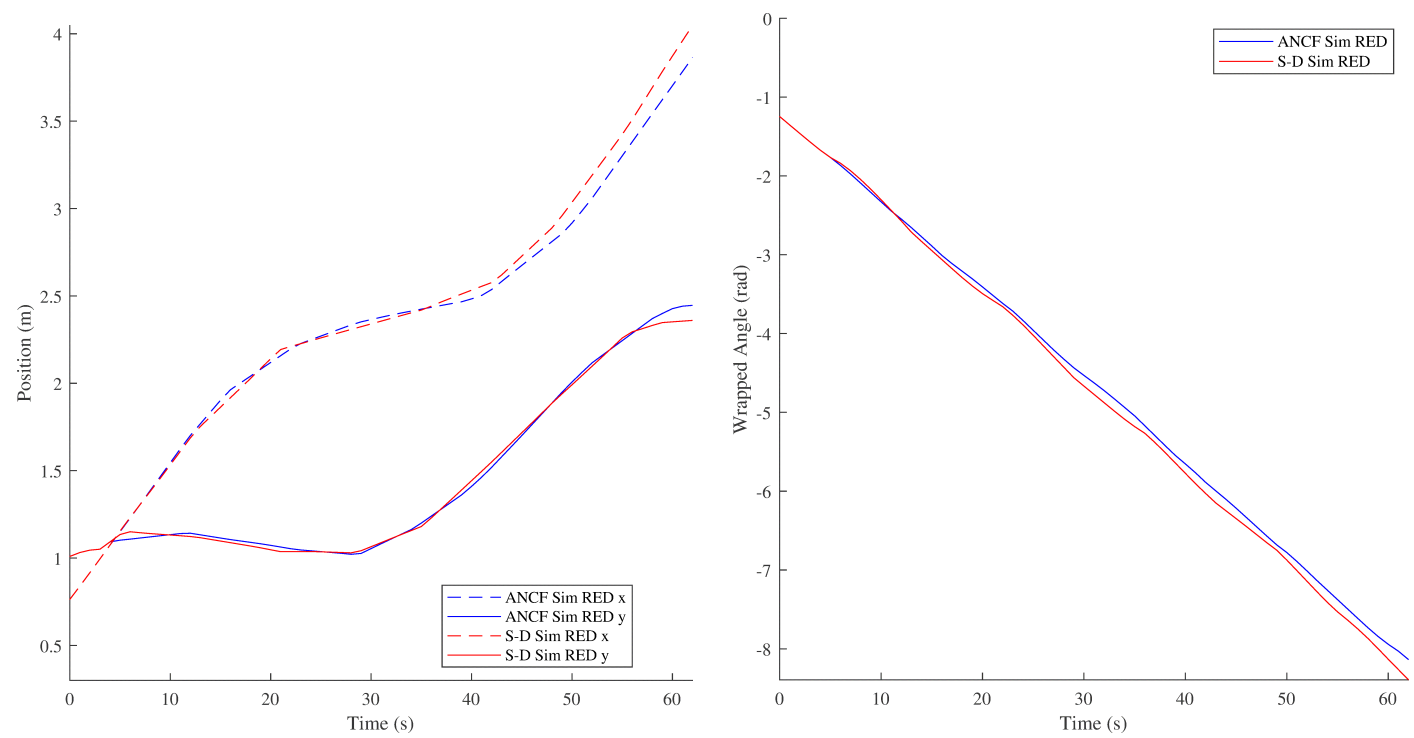

(a) Chaser platform positions during the sim- (b) Chaser platform pointing angles during ulated complete capture scenario post-capture the simulated complete capture scenario postphase. capture phase.
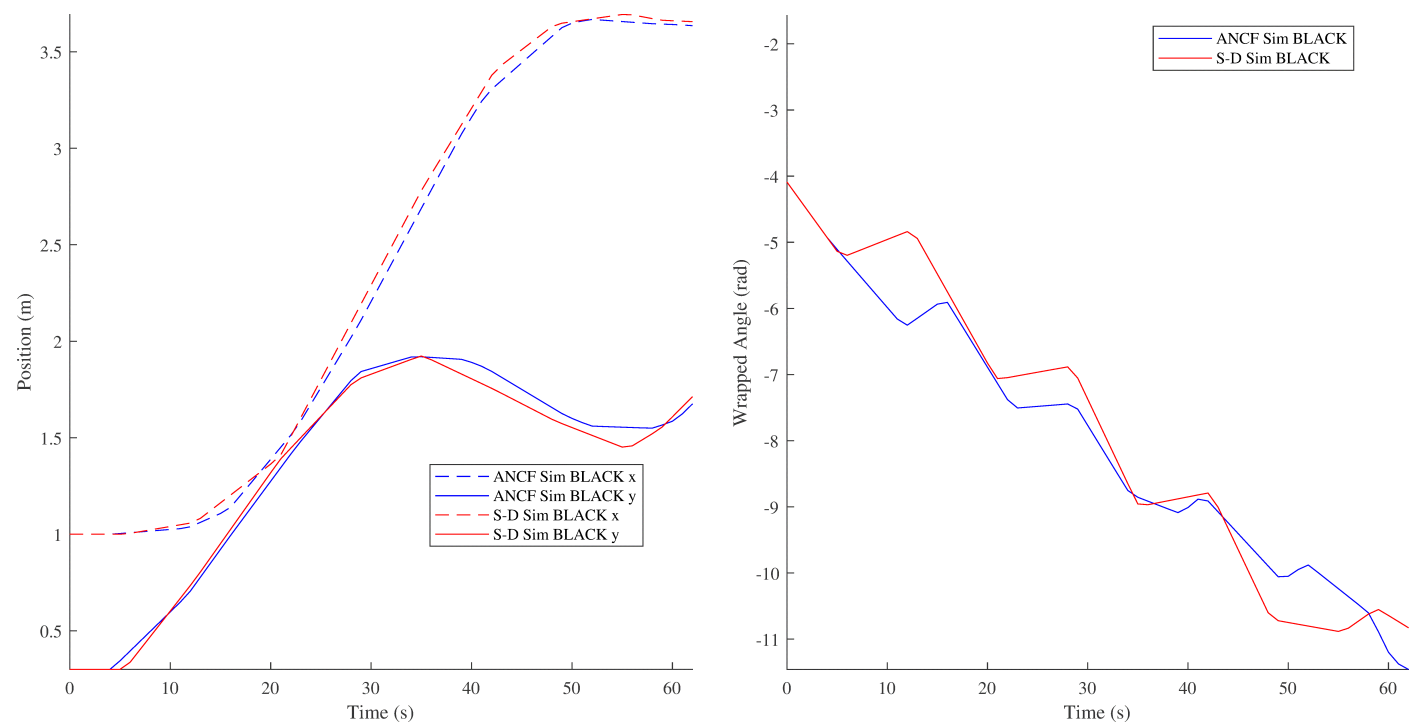

(c) Target platform positions during the sim- (d) Target platform pointing angles during ulated complete capture scenario post-capture the simulated complete capture scenario postphase.

capture phase.

Figure 4.4: Platform positions and pointing angles during the simulated planar complete capture scenario. 


\section{Chapter 5}

\section{Experimental Validation}

Chapter 5 presents both the tether deployment and complete capture scenario experiments. It is preformed in Carleton University's Spacecraft Robotics and Control Laboratory (SRCL) using the Spacecraft Operations Testbed (SPOT). The planar simulations discussed in Chap. 4.3 are compared to the experimental tether deployment data to determine the validity of the numerical models presented in this thesis.

\subsection{Introduction}

To gatherer experimental results, the SPOT facility along with both SPOT platforms were used. The tether deployment and complete capture scenario experiments were chosen such that they are experimentally replicable. The experiment consists of two spacecraft platforms placed on the table. The red platform represents the chaser and the black platform represents the target. The tether is initially coiled up and resting on the payload level of the chaser platform. In the setup phases of the experiment the platforms sit on the table and communicate with the PhaseSpace ground truth system system until a strong lock has been acquired. Once acquired, the platforms begin to float and are allotted 10 seconds to reach their respective initial positions on the table. Once there, the platforms spend 2 seconds holding their positions before the experiment begins. The experiment consists of an initial 15 seconds of coordinated platform movement before the tether is deployed and the TSS floats 60 seconds. The movement of the platforms has been chosen to create a difference in the angular rates of the two platforms, thereby emulating a non-ideal pose tracking maneuver.

Figure 5.1(a) shows the trajectory of the two platforms during the initial 15 seconds of the experiment. The target rotates in place, simulating debris arbitrarily drifting in space. The chaser orbit around the target in a circular motion and always faces it. When an opportunity for tether deployment is presented, that is a suitable 
surface on the target is aligned with the chaser, the tether is deployed. As seen in Fig. 5.1(b), tether deployment occurs when the chaser is roughly at the position $(1,1) \mathrm{m}$ on the table and the tether deployment payload and target impact panel are aligned. Post-deployment, both platforms will halt all controlled movement and the chaser only uses its thrusters to maintain its orientation aligned with the center of the target. In the post-capture stabilization phase once the tethered spacecraft system (TSS) has been established, both platforms will rotate about the barycentre of the TSS for 60 seconds, or until the TSS drifts too close to the edge of the table and the experiment is aborted, whichever comes first.
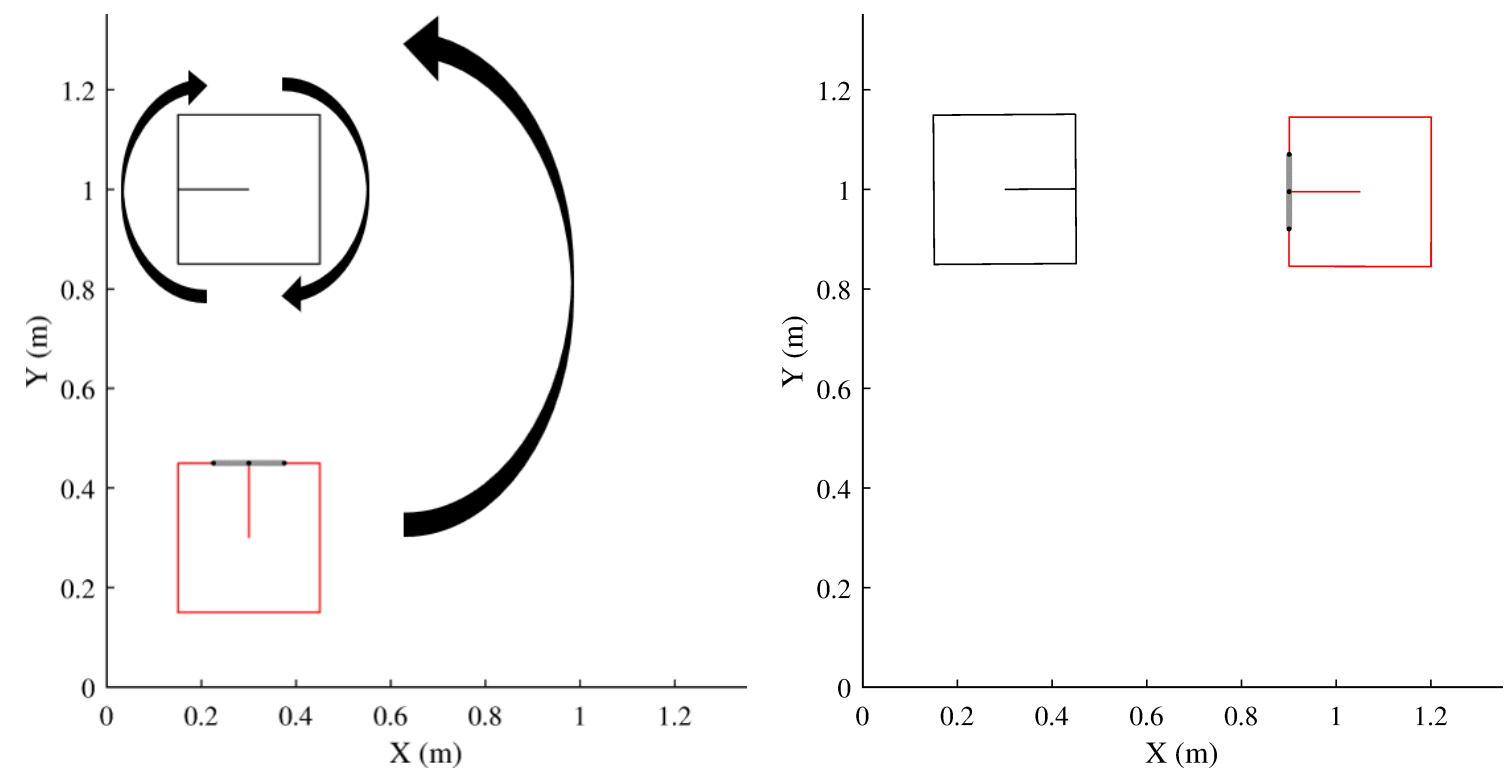

(a) Simulated platform movement during experi- (b) Simulated platform locations at the time of ment. tether deployment.

Figure 5.1: Experimental scenario SPOT platform movement.

Ten tether deployment experiments and a complete capture experiment were preformed. Platform and tether parameters for all experiments are presented in Table 5.1.

\subsection{Planar Tether Deployment Validation}

The experiment outlined in Chap. 5.1 was performed as described ten times. It was modified so that the experiment was halted 1 second after tether deployment as the post-capture phase was not relevant. In each experiment the chaser platform was 
Table 5.1: Laboratory experiment parameters.

\begin{tabular}{cccccc}
\hline \hline Parameter & Value & Parameter & Value & Parameter & Value \\
\hline$A, \mathrm{~m}^{2}$ & $7.85 \times 10^{-7}$ & $m_{b}, \mathrm{~kg}$ & $1.50 \times 10^{-3}$ & $L_{\text {main }}, \mathrm{m}$ & 0.28 \\
$\rho, \mathrm{kg} / \mathrm{m}^{3}$ & 1150 & $m_{\text {tether }}, \mathrm{kg}$ & $1.00 \times 10^{-4}$ & $L_{\text {sub }}, \mathrm{m}$ & 0.28 \\
$k, \frac{\mathrm{N}}{\mathrm{m}}$ & 175 & $m_{R E D}, \mathrm{~kg}$ & 21.23 & $I_{R E D}, \mathrm{kgm}^{2}$ & 0.29 \\
$c, \frac{\mathrm{Ns}}{\mathrm{m}}$ & 1.1 & $m_{B L A C K}, \mathrm{~kg}$ & 13.75 & $I_{B L A C K}, \mathrm{kgm}^{2}$ & 0.19 \\
$F_{\text {deployment }}, \mathrm{N}$ & 77 & & & & \\
\hline \hline
\end{tabular}

able to deploy the tether and successfully establish the TSS with the target platform. Each tether deployment trial was recorded using a slow-motion video camera at 480 frames per second. Node locations matching those of the planar simulations were marked on the experimental tether and their positions were manually recorded on each frame of the video at 0.1 second intervals. To correlate the experimental and planar simulation node positions a calibration ratio between a given pixel in the video frame and a corresponding position on the SPOT table was used. This calibration ratio was applied to the experimental node positions in order to visualize the experimental tether in the same inertial frame as the planar simulations.

The experimental, spring damper, and ANCF tether node positions are plotted for each time step of each trial. An example of these overlaid tether deployment plots for various points during an experiment are seen in Fig. 5.2. It is noted that the tethers are beginning approximately at the position $(0.9,1.0) \mathrm{m}$ of the SPOT table and deploy in the negative $x$-direction.

As seen in Fig. 5.2, there is a general agreement between the motion of the simulations and the experimental tethers, however, differences between the nodal positions of the two models and the experiment are nonnegotiable. To quantify these errors, the absolute residual of the tether node positions is used. It is expressed as:

$$
\mathbf{r e}_{i}=\left|\mathbf{s}_{i}-\mathbf{e x}_{i}\right|
$$

where $\mathbf{s}_{i}$ and $\mathbf{e x}_{i}$ represent the displacement of the $i^{\text {th }}$ tether node in simulation and experiment, respectively. 
For each time step of each experiment, the absolute residual of each tether node is calculated. As an example, the absolute residuals of the tether deployment of experiment 3 are shown in the box plots of Fig. 5.3. On each plot the ANCF model is in blue, the spring-damper model is in red, the central mark on the box indicates the median value, the bottom and top edges of the box indicate the $25^{\text {th }}$ and $75^{\text {th }}$ percentiles, respectively, the whiskers extend to the most extreme data points not considered outliers, and any data points considered to be outliers are plotted individually using the "+" symbol. Plotting the data in a box plot allows for easy visualization of which nodes of the tether have a wide range in their absolute residuals during the deployment.

The absolute residuals of each node, averaged across all ten experiments, for both the massless spring-damper and ANCF numerical models are shown in Table 5.2. Individual box plots of the absolute residuals for each tether and the junction of each experiment, omitting experiment 3, are found in App. A.

Overall the average absolute residual of every node for both the spring-damper and ANCF models lies at only a few centimeters of error. It can be seen that on average the spring-damper model error is less for the bullets and for the nodes close to the tip of the sub-tethers. While the ANCF model error is less at the junction, the sub-tether nodes closest to it, and the main tether. Additionally, the error found in the ANCF model is on average higher than that of the spring-damper model. This is explained due to artificial errors that are inherently introduced into the model. Each ANCF tether element must always remain it's total unstretched length when no forces are acting on it. Due to this, when the end-points of the tether are initially defined, the shape function at that state will dictate how the tether is folded. As an example shown by Fig. 5.4, when in its initial state, the tether junction and attachment point to the SPOT platform are halfway between the two bullets, with the two bullets directly above and below. The ANCF shape function initially folds the tether along this axis, and in order to accommodate the full unstretched length of the tether it will create an "S" shape. While unfolding, the nodes of the ANCF tether are not as close to the nodes of the spring-damper simulation which are evenly spaced along the edge of the platform with excess tether folded into a single point at the median 
Table 5.2: Average relative residuals of the spring-dapmer and ANCF models.

\begin{tabular}{|c|c|c|c|c|}
\hline \multirow{2}{*}{$\begin{array}{c}\text { Axis } \\
\text { Model }\end{array}$} & \multicolumn{2}{|c|}{$\mathbf{x}$} & \multicolumn{2}{|c|}{$\mathbf{y}$} \\
\hline & S-D & ANCF & S-D & ANCF \\
\hline Node & \multicolumn{4}{|c|}{1} \\
\hline Sub-Tether 1 & $3.09 \mathrm{~cm}$ & $3.46 \mathrm{~cm}$ & $1.45 \mathrm{~cm}$ & $1.31 \mathrm{~cm}$ \\
\hline Sub-Tether 2 & $2.89 \mathrm{~cm}$ & $3.91 \mathrm{~cm}$ & $1.36 \mathrm{~cm}$ & $1.44 \mathrm{~cm}$ \\
\hline Main Tether & $2.25 \mathrm{~cm}$ & $1.27 \mathrm{~cm}$ & $0.40 \mathrm{~cm}$ & $0.59 \mathrm{~cm}$ \\
\hline Node & \multicolumn{4}{|c|}{2} \\
\hline Sub-Tether 1 & $2.92 \mathrm{~cm}$ & $3.04 \mathrm{~cm}$ & $1.52 \mathrm{~cm}$ & $1.82 \mathrm{~cm}$ \\
\hline Sub-Tether 2 & $3.15 \mathrm{~cm}$ & $3.08 \mathrm{~cm}$ & $1.16 \mathrm{~cm}$ & $1.32 \mathrm{~cm}$ \\
\hline Main Tether & $2.14 \mathrm{~cm}$ & $1.36 \mathrm{~cm}$ & $0.36 \mathrm{~cm}$ & $0.53 \mathrm{~cm}$ \\
\hline Node & \multicolumn{4}{|c|}{3} \\
\hline Sub-Tether 1 & $2.88 \mathrm{~cm}$ & $3.39 \mathrm{~cm}$ & $1.69 \mathrm{~cm}$ & $2.19 \mathrm{~cm}$ \\
\hline Sub-Tether 2 & $3.46 \mathrm{~cm}$ & $3.16 \mathrm{~cm}$ & $1.82 \mathrm{~cm}$ & $2.17 \mathrm{~cm}$ \\
\hline Main Tether & $1.36 \mathrm{~cm}$ & $1.00 \mathrm{~cm}$ & $0.30 \mathrm{~cm}$ & $0.32 \mathrm{~cm}$ \\
\hline Node & \multicolumn{4}{|c|}{4} \\
\hline Sub-Tether 1 & $2.92 \mathrm{~cm}$ & $3.32 \mathrm{~cm}$ & $1.75 \mathrm{~cm}$ & $1.96 \mathrm{~cm}$ \\
\hline Sub-Tether 2 & $3.45 \mathrm{~cm}$ & $3.06 \mathrm{~cm}$ & $3.12 \mathrm{~cm}$ & $2.36 \mathrm{~cm}$ \\
\hline Main Tether & $0.60 \mathrm{~cm}$ & $0.49 \mathrm{~cm}$ & $0.20 \mathrm{~cm}$ & $0.12 \mathrm{~cm}$ \\
\hline Node & \multicolumn{4}{|c|}{5} \\
\hline Sub-Tether 1 & $2.75 \mathrm{~cm}$ & $2.68 \mathrm{~cm}$ & $1.72 \mathrm{~cm}$ & $0.97 \mathrm{~cm}$ \\
\hline Sub-Tether 2 & $3.08 \mathrm{~cm}$ & $2.44 \mathrm{~cm}$ & $2.16 \mathrm{~cm}$ & $1.60 \mathrm{~cm}$ \\
\hline Main Tether & $0.00 \mathrm{~cm}$ & $0.00 \mathrm{~cm}$ & $0.00 \mathrm{~cm}$ & $0.00 \mathrm{~cm}$ \\
\hline Node & \multicolumn{4}{|c|}{ Junction } \\
\hline & $2.08 \mathrm{~cm}$ & $1.55 \mathrm{~cm}$ & $0.46 \mathrm{~cm}$ & $0.60 \mathrm{~cm}$ \\
\hline
\end{tabular}

between the bullet nodes. This difference in tether folding results in artificial errors being introduced to the average absolute residuals of the ANCF model early on in the deployment of the tether.

Other errors associated with the tether deployment experiment are air resistance and gravitational force acting in the $z$-axis. Air resistance is not accounted for in the simulated planar environment because it is assumed to have a minimal effect on the tether dynamics due to their very small surface area. However, in practice there will be a very small change in the tether dynamics due to the resisting force. In simulation gravitational forces are not accounted for as the simulated planar environment does not have a third dimension for the force to act in. In experiment, the 
gravitational forces were found to cause the tether and bullets to drop in height by a few centimeters. The existence of a third dimension and constant force in experiment changes tether flight dynamics, but since the force cannot be removed and effects the experiments equally all results would have the same amount of induced error.

Considering all of the errors, the average absolute residuals are found to be low and confirm that although both numerical models are capable of describing the motion of the experimental tethers. The ANCF model is better at describing the motion of the junction, nearby nodes, and the main tether, while the spring-damper model is better at describing the motion of the tether ends and nodes closest to them. The ANCF model is better at describing the motion of the tether once it has been mostly deployed as seen by Fig. 5.2(c), while the spring-damper model is better at describing the early deployment motion of the tether as seen by Fig. 5.2(a). However, it should be noted that this conclusion is influenced by the artificial errors of the initial tether positions in the ANCF model. Overall both tether models can be considered adequate at describing the motion of the tethers during deployment. The spring-damper model has a maximum average absolute residual error of $3.46 \mathrm{~cm}$ in the $x$-axis and $3.12 \mathrm{~cm}$ in the $y$-axis across ten experiments, and the ANCF model has a maximum average absolute residual error of $3.91 \mathrm{~cm}$ in the $x$-axis and $2.36 \mathrm{~cm}$ in the $y$-axis across ten experiments. These errors are across a constant flight distance of $53.8 \mathrm{~cm}$ in the $x$-axis, and average $5 \mathrm{~cm}$ displacement in the $y$-axis.

If these errors were scaled up to the on-orbit complete capture scenario presented in Chap. 2.3 then they would result in a worst-case scenario of $2.18 \mathrm{~m}$ of error along the deployment direction of the tether. Although this is a sizable error, it can easily be compensated for by ensuring that the distance between the chaser and debris is less than the unstretched length of the tether. It is concluded that the numerical models are cable of accurately modeling the sub-tether configuration and the associated errors would be manageable in a real-world scenario.

\subsection{Complete Capture Scenario Results}

To demonstrate the complete capture scenario in a planar environment the experimental trajectories of the chaser and target platforms are compared to their simulated 
counterparts. In simulation once the TSS has been established, Hovell's [46] springdamper model was used as outlined in Chap. 4.2.6. The attachment points between both sub-tether bullets and the target platform are the same as shown in Figs. 4.2(l) and 4.3(1) of Chap. 4.3.

The platforms positions in the experiment are shown in Figs. 5.5(a) and 5.5(c) while their pointing wrapped pointing angles are shown in Figs. 5.5(b) and 5.5(d). It is important to note that these plots omit the initial setup time of the experiment as it is controlled movement and begin just before tether deployment.

The experimental and simulation TSS platform trajectories have the same general direction of movement and pointing angles post-capture. However, beyond the similar motion a sleight discrepancy can be seen between the experimental and simulation values when the sub-tethers are in tension, as seen during the 40-70 second time span of Figs 5.5(b) and 5.5(d). The angle that the target achieves in simulation is much larger than in the experiment. This discrepancy was concluded to be due to several factors, including an imperfect experimentally determined spring constant and damping ratio of the tether, the platform velocities being lower than expected, air resistance on the platforms, and the tether not contacting at the maximum unstretched length.

The full capture scenario was only run for a total of 70 seconds due to the platforms approaching the limits of the SPOT table. If it were to be run longer it could be expected that the plot trends would be similar to those seen in Fig. 4.4 of Chap. 4.5.

This experiment was preformed to demonstrate, for the first time, a complete capture scenario of the tethering to an uncooperative target with differing angular rates using the novel tether configuration. It is concluded that a complete capture scenario in a planar environment using the novel tether configuration is possible. A video of the complete capture scenario can be accessed at https://tinyurl.com/ yy9faazm. 


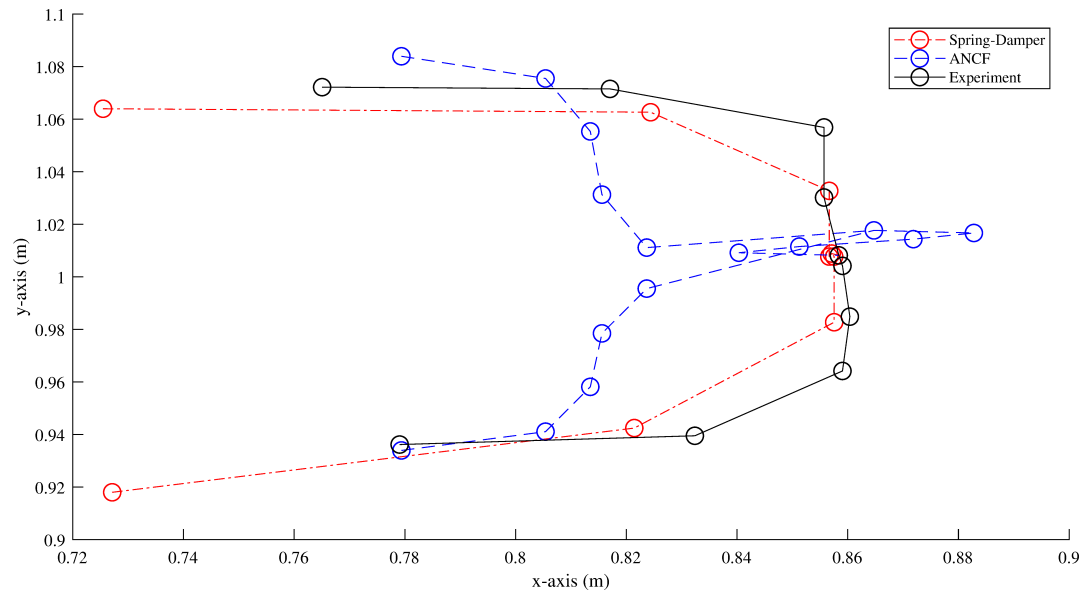

(a) Experiment 3 overlaid tether deployment at 0.2 seconds

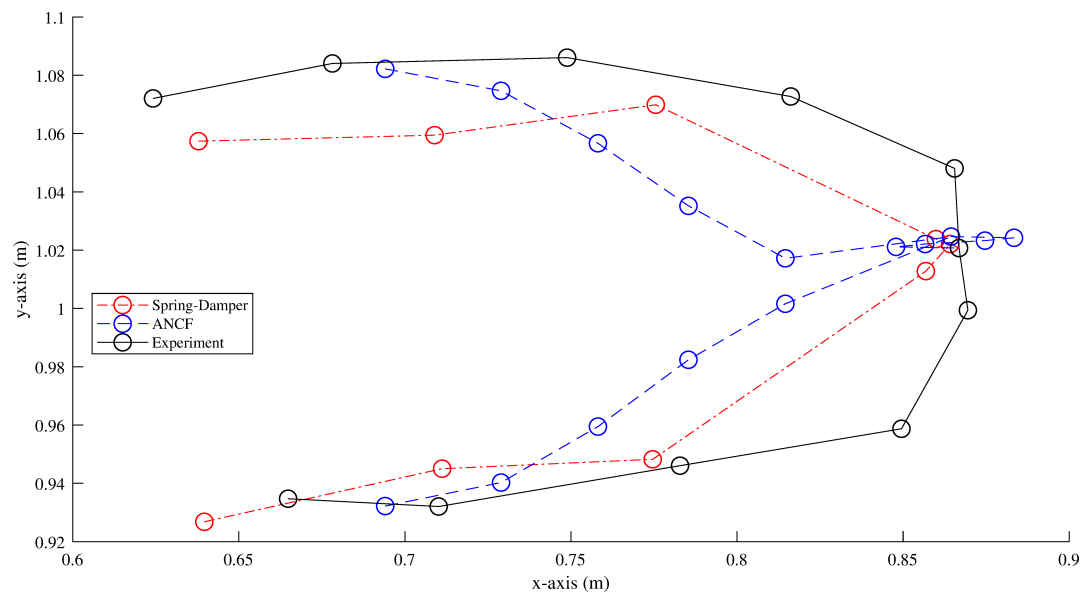

(b) Experiment 3 overlaid tether deployment at 0.4 seconds

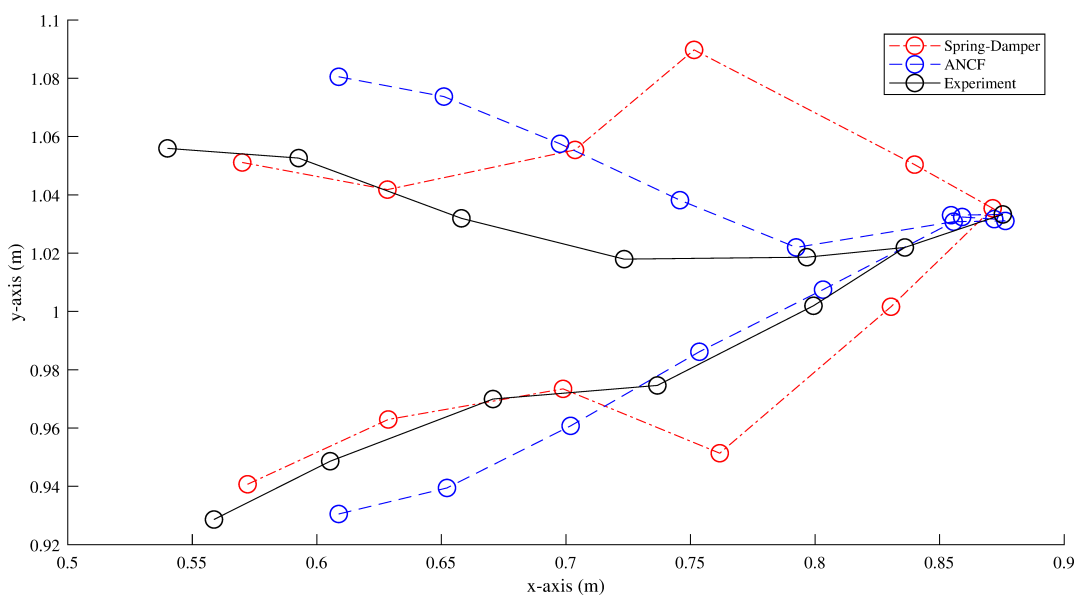

(c) Experiment 3 overlaid tether deployment at 0.6 seconds 


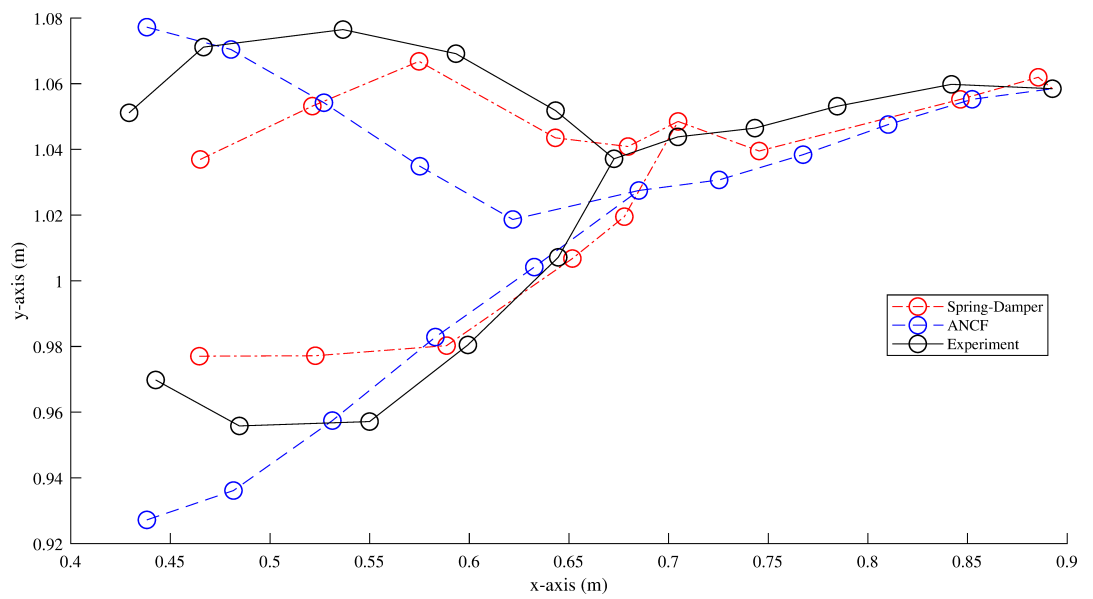

(d) Experiment 3 overlaid tether deployment at 1.0 seconds

Figure 5.2: Spring-damper, ANCF, and experimental tether deployment of trial 2 at various time steps. 


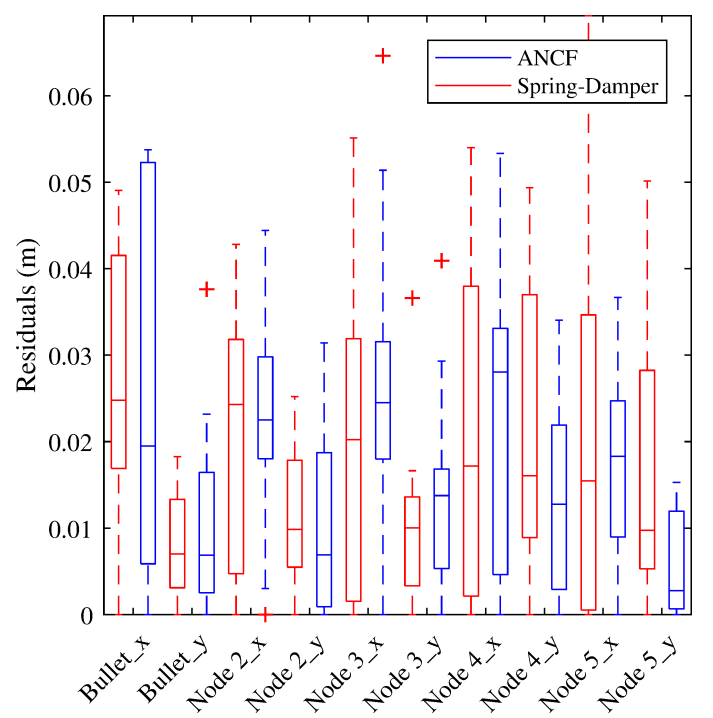

(a) Experiment 3 Sub-Tether 1 Residuals

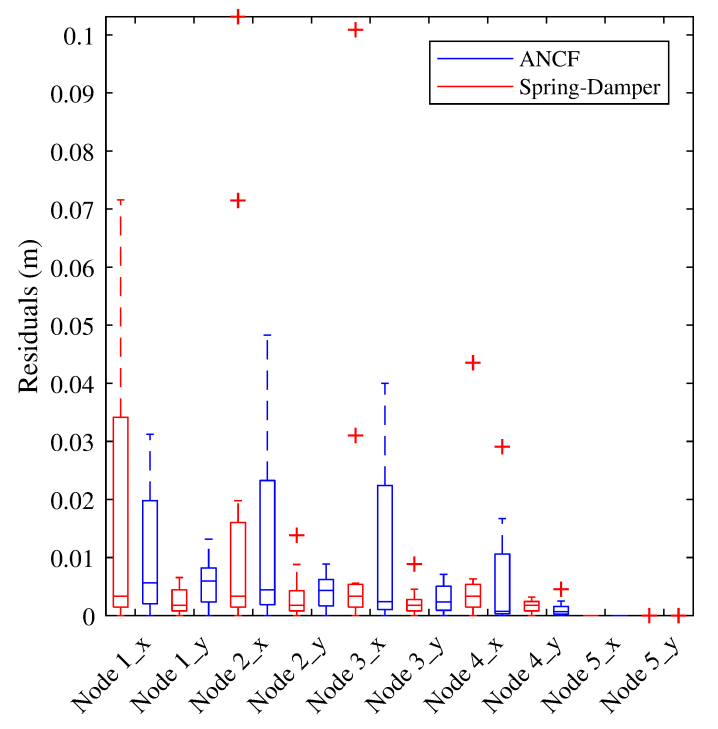

(c) Experiment 3 Main Tether Residuals

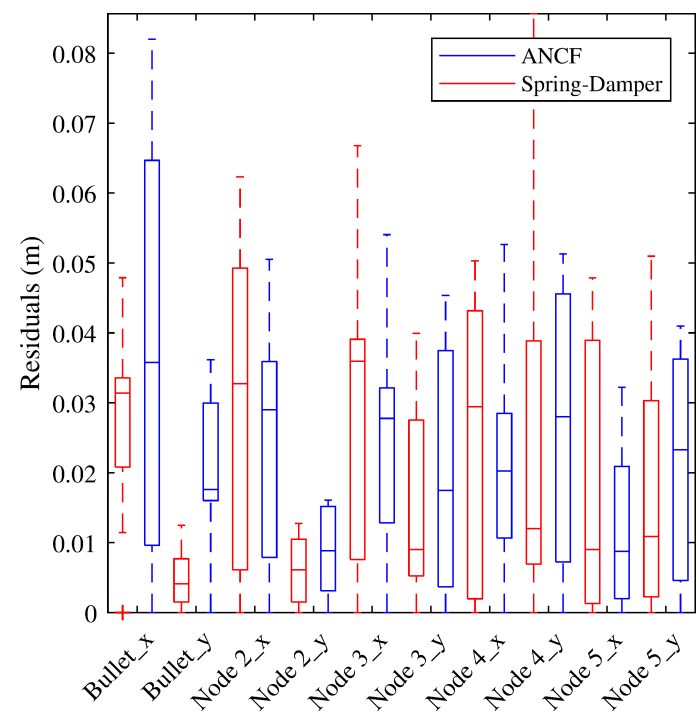

(b) Experiment 3 Sub-Tether 2 Residuals

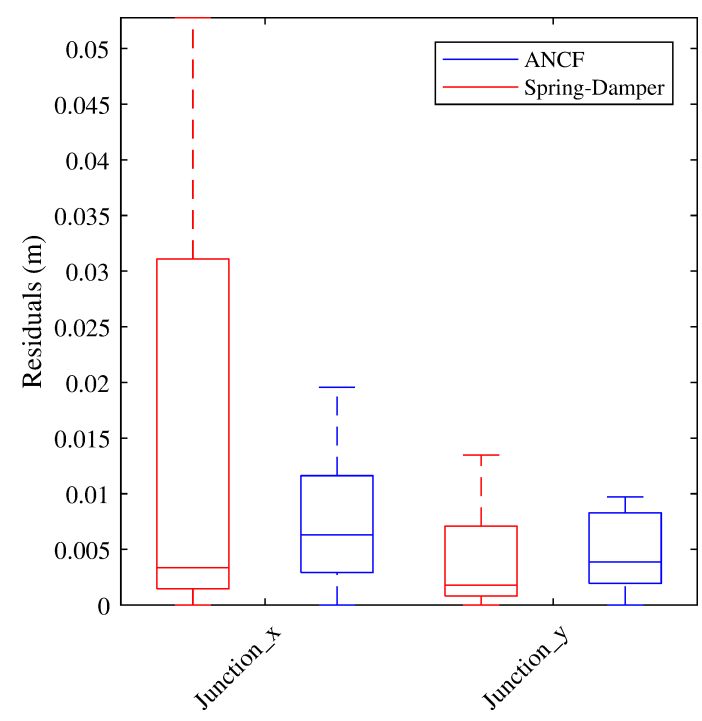

(d) Experiment 3 Junction Residuals

Figure 5.3: Experiment 3 individual tether node absolute residuals over the duration of tether deployment. 


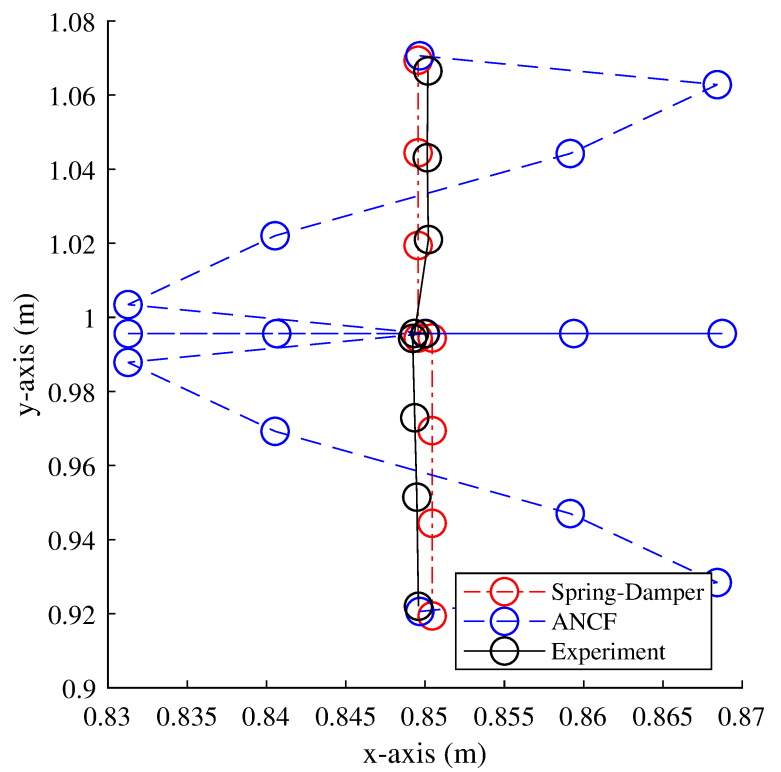

Figure 5.4: Spring-damper, ANCF, and experimental tether nodes initial positions. 

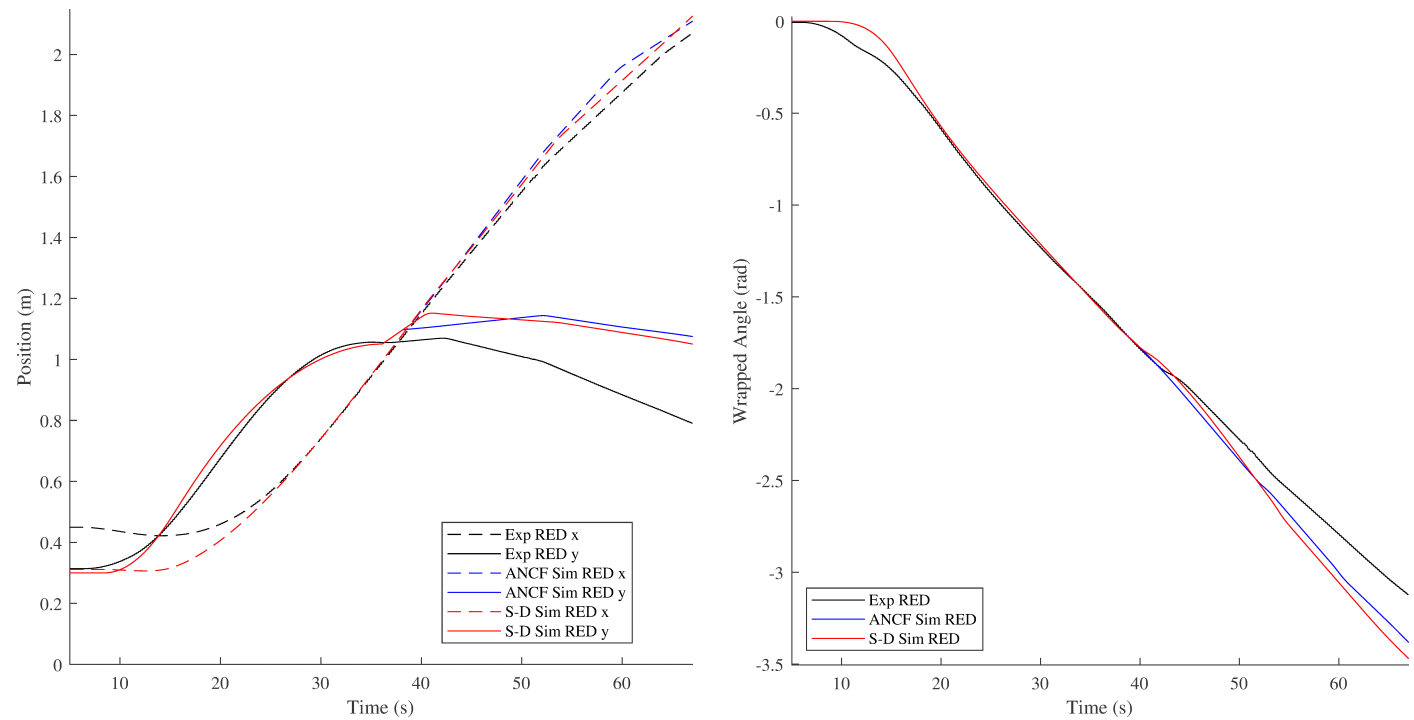

(a) Chaser platform positions during the com- (b) Chaser platform pointing angles during the plete capture scenario. complete capture scenario.
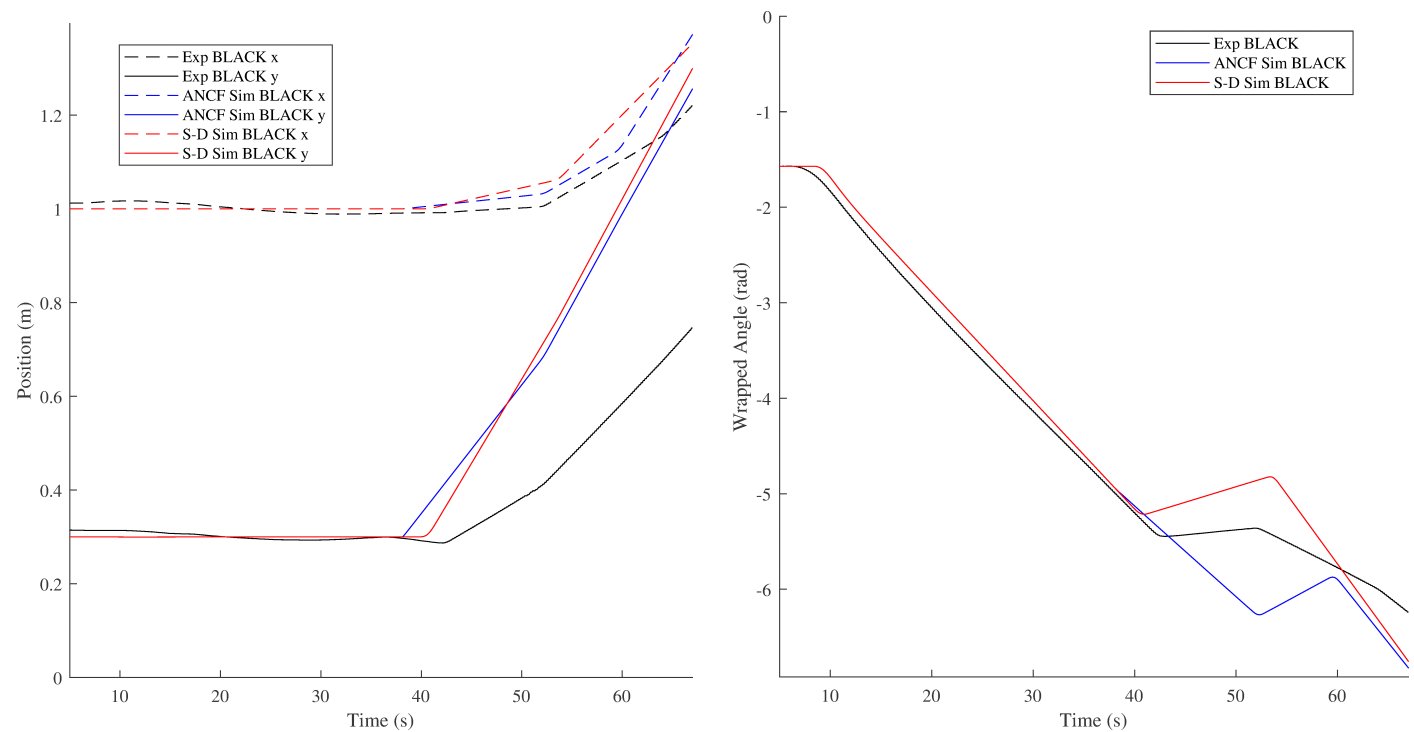

(c) Target platform positions during the complete capture scenario.

(d) Target platform pointing angles during the complete capture scenario.

Figure 5.5: Platform positions and pointing angles during the planar complete capture scenario. 


\section{Chapter 6}

\section{Conclusion}

This chapter summarizes the motivation, research methodology, and results previously presented in this work. The impact, contributions, and the practicality of this research on the field of space debris removal is discussed alongside future work that can be done to build upon this research.

\subsection{Thesis Summary}

The threat of space debris is a growing concern for all spacefaring organizations of the world and those who benefit from the space-based services provided to them. Known as the Kessler Syndrome, collisions in orbit have cascading effects because even after a collision, resulting debris remains in orbit. Depending on the objects involved, each orbital collision can create hundreds to thousands of new debris pieces. Newly created debris will continue to orbit, each posing the threat of causing future collisions and therefore greatly increasing the overall risk of the orbital environment. The first solution to this problem is to restrict the rate of new orbital debris growth. Mitigation measures have been enacted, with cooperating countries implementing a 25 year limit to spacecraft missions. The second solution to this problem is the active removal of high-risk debris from orbit. It is estimated that the removal of $5-10$ of these objects per year will be sufficient in halting the growth of the orbital debris population.

This thesis focuses on the active debris removal concept. Hovell and Ulrich [8-11] first proposed a novel tether design for the improved detumbling capabilities of a flexible tethered spacecraft system and demonstrated its improved post-capture target stabilization capabilities. This thesis specifically focuses on the deployment phase of the novel tether, presenting two numerical models which are used to replicate its 
motion in simulation, and a numerical model for determining the simultaneous multibody contact dynamics of the tether and target object. Modeling the deployment phase of the novel tether, and validating the models, is important as it gives initial insight into how the tether would behave in an orbital mission scenario. There is a multitude of research focused on the deployment and contact dynamics of nets in an orbital environment, however, they all use a single tether to establish the tethered spacecraft system. This work bridges the gap between dynamical net models and the novel tether configuration. This is shown firstly by validating the numerical models used to simulate the deployment of the tethers. Additionally, a complete capture scenario using the novel tether was presented for the first time.

The main contribution of this thesis is the validation of the novel tether design over the complete capture scenario. First, two tether deployment models were presented in order to numerically model the expected motion of the tether during deployment. These models were implemented alongside a contact dynamics model and Hovell's [46] post-capture spring-damper tether model in order to numerically simulate the complete capture scenario. The first simulation was comprised of two spacecraft, the deployed tether, and incorporated the orbital dynamics and gravity gradient forces of lower Earth orbit. The second simulation was in a planar environment replicating Carleton University's Spacecraft Operations Testbed. The planar numerical models were experimentally validated. To accomplish this, a tether deployment system, tether bullets, and a platform impact panel were developed and incorporated into the lab. Using the laboratories experimental platforms, both the tether deployment and complete capture scenarios were experimentally preformed and the results were used to validate the numerical tether models presented in this thesis. Discrepancies between the simulations and experiments were found to be small. Unmodeled effects

present in the laboratory environment such as friction, drag, and signal noise are attributed to these discrepancies.

\subsection{Contributions to the Field of Space Debris Removal}

The significance of this work is demonstrated by the publication: 
Stadnyk, K., and Ulrich, S., Validating the Deployment of a Novel Tether Design for Net-Based Orbital Debris Removal Missions, 30 ${ }^{\text {th }}$ AIAA/AAS Space Flight Mechanics Meeting, Orlando, FL, 6-10 Jan, 2020.

\subsection{Practicality of this Work}

This work presented the complete capture scenario of the sub-tether design. It focused on the addition of the tether deployment and contact phases to establish a tethered connection between the chaser and the target. In doing so, only the tether configurations were considered and there was no method of anchoring the tether to the target. That is, there is no net modeled at the end of the tether.

The four bullet nodes at the end of the sub-tethers act as harpoons, however, in practice the harpoon method is not considered piratical. In the single tether configuration, a harpoon is a practical method of establishing the tether between the chaser and target. However, in the sub-tether configuration multiple tethered harpoons would need to simultaneously be launched and contact the target. This greatly increases the number of failure points of the system, and is further complicated when the target is tumbling. In the event that one or more of the sub-tethers do not contact the target, they cannot be reeled in and redeployed. This would compromise the stabilization process and ultimately cause the failure of the mission. The use of harpoons with the sub-tether configuration is not feasible.

A net attached to the end of the sub-tethers is a more suitable method of capturing the target. This is because nets are capable of ensnaring its target regardless of the targets orientation. In addition, nets are generally much larger than the target when fully deployed, allowing it to not require pinpoint accuracy in order to hit its target. The sub-tether configuration interfaces well with a net as the sub-tethers can be equally spaced out and attached at the desired locations. Although a net is the preferred sub-tether configuration attachment method, it has been omitted from this work. Indeed, the planar experimental environment does not allow for a net to be used. In keeping this work focused solely on validating the tether deployment and complete capture scenario of the novel tether design the modeling of a net for the on-orbit simulation was outside of the scope of this work. 


\subsection{Recommendations for Future Work}

Future work should focus on the modeling of a net attached to the end of the subtethers which is integrated into the deployment and contact phases of the numerical simulations. Experimentally validating these updated numerical simulations should be done in a microgravity environment, such as one provided on aircraft capable of preforming parabolic flight maneuvers. The deployment of the tethered-net should be done using a stored mechanical energy device such as a set of springs to avoid a compressed gas used to propel the bullets from affecting the flight of the net and tethers. An orbital demonstrator mission should be preformed in order to validate this technology. Lastly, the capture scenario phases can be expanded upon to include more complex rendezvous maneuvers with the target, and possible maneuvers to despin the TSS while maintaining control of the target. 


\section{Bibliography}

[1] Kessler, D. J. and Cour-Palais, B. G., "Collision Frequency of Artificial Satellites: The Creation of a Debris Belt," Journal of Geophysical Research, Vol. 83, No. A6, 1978, pp. 2637-2646.

[2] European Space Agency, "Space Debris by the Numbers," 2019.

[3] NASA Orbital Debris Program Office, "Orbital Debris Quarterly News," Vol. 23, No. 1 \& 2, 2019, pp. 1-14.

[4] Pardini, C. and Anselmo, L., "Assessment of the consequences of the Fengyun$1 \mathrm{C}$ breakup in low Earth orbit," Advances in Space Research, Vol. 44, No. 5, 2009, pp. 545-557.

[5] Kelso, T., "Analysis of the Iridium 33-Cosmos 2251 Collision," Tech. rep., 2009.

[6] Krag, H., "The Inter-Agency Space Debris Coordination Committee - An overview of the IADC annual activities," Harwell Oxford, UK, 2016, p. 18.

[7] Liou, J.-C., "An active debris removal parametric study for LEO environment remediation," Advances in Space Research, Vol. 47, No. 11, 2011, pp. 1865-1876.

[8] Hovell, K. and Ulrich, S., "Attitude Stabilization of an Unknown and Spinning Target Spacecraft Using a Visco-Elastic Tether," 13th Symposium on Advanced Space Technologies in Robotics and Automation, Noordwijk, The Netherlands, 2015 .

[9] Hovell, K. and Ulrich, S., "Attitude Stabilization of an Uncooperative Spacecraft in an Orbital Environment using Visco-Elastic Tethers," AIAA Guidance, Navigation, and Control Conference, San Diego, CA, 2016.

[10] Hovell, K. and Ulrich, S., "Experimental Validation for Tethered Capture of Spinning Space Debris," AIAA Guidance, Navigation, and Control Conference, Grapevine, TX, 2017.

[11] Hovell, K. and Ulrich, S., "Postcapture Dynamics and Experimental Validation of Subtethered Space Debris," AIAA Journal of Guidance, Control, and Dynamics, Vol. 41, No. 2, 2018, pp. 519-525.

[12] Shan, M., Guo, J., and Gill, E., "Review and Comparison of Active Space Debris Capturing and Removal Methods," Progress in Aerospace Sciences, Vol. 80, 2015, pp. 18-32. 
[13] Phipps, C., "A laser-optical system to re-enter or lower low earth orbit space debris," Acta Astronaut, Vol. 93, 2014, pp. 418-429.

[14] ESA, "Magnetic Space Tug Could Target Dead Satellites," 2017.

[15] Persson, S. M. and Sharf, I., "Ground-Based Experiments Towards the Interception of Non-Cooperative Space Debris with a Robotic Manipulator," IEEE International Conference on Intelligent Robots and Systems, Hamburg, Germany, 2015.

[16] Shah, S. V., Sharf, I., and Misra, A. K., "Reactionless Path Planning Strategies for Capture of Tumbling Objects in Space Using a Dual-Arm Robotic System," AIAA Guidance, Navigation, and Control Conference, Boston, MA, 2013.

[17] Chiesa, A. and Alberto, F., "Enabling technologies for active space debris removal: the cadet project," Paris, France, 2014.

[18] Canadian Space Agency, "Canadarm, Canadarm2, and Canadarm3 A comparative table," 2019.

[19] Ellery, A., "Tutorial Review on Space Manipulators for Space Debris Mitigation," Robotics, Vol. 8, No. 2, 2019, pp. 1-52.

[20] Biesbroek, R., "The e.Deorbit CDF Study," Tech. rep., Montréal, Canada, 2012.

[21] Chiesa, A., Fossati, F., Gambacciani, G., and Pensavalle, E., "Enabling Technologies for Active Space Debris Removal: The Cadet Project," Space Safety is No Accident, Springer, 2015, pp. 29-38.

[22] Yoshida, K. and Nakanichi, H., "The TAKO (Target Collaborativise) Flyer: a New Concept for Future Satellite Servicing," 6th International Symposium on Artificial Intelligence and Robotics \& Automation in Space, Quebec, 2001.

[23] Williams, P., "Optimal Orbit Transfer with Electrodynamic Tether," Journal of Guidance, Control, and Dynamics, Vol. 28, No. 2, 2005, pp. 369-372.

[24] Ohkawa, Y., Kawamoto, S., Nishida, S., and Kitamura, S., "Research and Development of Electrodynamic Tethers for Space Debris Mitigation," Transactions of the Japan Society for Aeronautical and Space Sciences, Space Technology Japan, Vol. 7, No. ists26, 2009, pp. 5-10.

[25] "Japan goes fishing for space junk but 700-metre 'tether' fails," 2017.

[26] Wormnes, K., Le Letty, R., Summerer, L., Schonenborg, R., Dubois-Matra, O., Luraschi, E., Cropp, A., Krag, H., and Delaval, J., "ESA Technologies for Space Debris Remediation," 6th European Conference on Space Debris, Darmstadt, Germany, 2013. 
[27] Dudziak, R., Tuttle, S., and Barraclough, S., "Harpoon Technology Development for the Active Removal of Space Debris," Advances in Space Research, Vol. 56, No. 3, 2015, pp. 509-527.

[28] Forshaw, J. L., Aglietti, G. S., Navarathinam, N., Kadhem, H., Salmon, T., Pisseloup, A., Joffre, E., Chabot, T., Retat, I., Axthelm, R., Barraclough, S., Ratcliffe, A., Bernal, C., Chaumette, F., Pollini, A., and Steyn, W. H., "RemoveDEBRIS: An in-orbit active debris removal demonstration mission," Acta Astronautica, Vol. 127, 2016, pp. 448-463.

[29] Zhao, Y., Huang, P., and Zhang, F., "Capture Dynamics and Net Closing Control for Tethered Space Net Robotics," Journal of Guidance, Control, and Dynamics, Vol. 42, No. 1, 2019, pp. 199-208.

[30] Botta, E. M., Sharf, I., Misra, A. K., and Teichmann, M., "On the Simulation of Tether-Nets for Space Debris Capture with Vortex Dynamics," Acta Astronautica, Vol. 123, 2016, pp. 91-102.

[31] Botta, E. M., Sharf, I., and Misra, A. K., "Contact Dynamics Modeling and Simulation of Tether Nets for Space-Debris Capture," Journal of Guidance, Control, and Dynamics, Vol. 40, No. 1, 2017, pp. 110-123.

[32] Benvenuto, R. and Lavagna, M., "Flexible Capture Devices for Medium To Large Debris Active Removal: Simulations Results To Drive Experiments," 12th Symposium on Advanced Space Technologies in Automation and Robotics, Noordwijk, The Netherlands, 2013.

[33] Benvenuto, R. and Lavagna, M., "Net Capturing of Tumbling Space Debris: Contact Modelling Effects on the Evolution of the Disposal Dynamics," 13th Symposium on Advanced Space Technologies in Automation and Robotics, Noordwijk, The Netherlands, 2015.

[34] Benvenuto, R., Lavagna, M. R., and Salvi, S., "Multibody Dynamics Driving GNC and System Design in Tethered Nets for Active Debris Removal," Advances in Space Research, Vol. 58, No. 1, 2016, pp. 45-63.

[35] Shan, M., Guo, J., and Gill, E., "Deployment dynamics of tethered-net for space debris removal," Acta Astronautica, Vol. 132, 2017, pp. 293-302.

[36] Medina, A., Cercòs, L., Stefanescu, R., Benvenuto, R., Lavagna, M., Gonzalez, I., Rodriguez, N., and Ormnes, K., "Capturing Nets for Active Debris Removal: A Follow-Up on Microgravity Experiment Design To Validate Flexible Dynamic Models," 13th Symposium on Advanced Space Technologies in Automation and Robotics, Noordwijk, The Netherlands, 2015. 
[37] Cercos, L., Stefanescu, R., Medina, A., Benvenuto, R., Lavagna, M., Gonzalez, I., Rodriguez, N., and Wormnes, K., "Validation of a Net Active Debris Removal Simulator Within Parabolic Flight Experiment," International Symposium on Artificial Intelligence, Robotics and Automation in Space, Montreal, Quebec, Canada, 2014.

[38] Lavagna, M. R., Armellin, R., Bombelli, A., Benvenuto, R., and Carta, R., "Debris Removal Mechanism Based on Tethered Nets," International Symposium on Artificial Intelligence, Robotics and Automation in Space, Turin, Italy, 2012.

[39] Shan, M., Guo, J., Gill, E., and Gołębiowski, W., "Validation of Space Net Deployment Modeling Methods Using Parabolic Flight Experiment," Journal of Guidance, Control, and Dynamics, Vol. 40, No. 12, 2017, pp. 3319-3327.

[40] Benvenuto, R., Salvi, S., and Lavagna, M., "Dynamics analysis and GNC design of flexible systems for space debris active removal," Acta Astronautica, Vol. 110, 2015, pp. 247-265.

[41] Shan, M., Net deployment and contact dynamics of capturing space debris objects, Ph.D. thesis, 2018.

[42] Liu, L., Shan, J., Ren, Y., and Zhou, Z., "Deployment dynamics of throw-net for active debris removal," 65th International Astronautical Congress, 2014.

[43] Mankala, K. K. and Agrawal, S. K., "Dynamic Modeling and Simulation of Satellite Tethered Systems," Vol. 127, No. April, 2005, pp. 144-156.

[44] Shuai, G., Yong, Y., Xiaofeng, S., and Yuhao, S., Dynamic Simulation of Fishing Net Based on Cubic B-Spline Surface., 2012.

[45] J., C. and Michael, G., "Deployment and Retrieval Simulation Of A Single Tether Satellite Systems," System Theory, 1988., Proceedings of the Twentieth Southeastern Symposium, Charlotte, NC, USA, 1988, pp. 657-660.

[46] Hovell, K., Detumbling Space Debris Using Tethers, Ph.D. thesis, Carleton University, 2017.

[47] Hughes, P. C., Spacecraft Attitude Dynamics, Dover Publications, Mineola, NY, 2004.

[48] Shabana, A. A., "Definition of the Slopes and the Finite Element Absolute Nodal Coordinate Formulation," Multibody System Dynamics, Vol. 1, No. 3, 1997, pp. 339-348.

[49] Shabana, A. A., Hussien, H., and Escalona, J., "Application of the Absolute Nodal Coordinate Formulation to Large Rotation and Large Deformation Problems," Journal of Mechanical Design, Vol. 120, No. 2, 1998, pp. 188-195. 
[50] Berzeri, M. and Shabana, A. A., "Development of simple models for the elastic forces in the absolute nodal co-ordinate formulation," Journal of Sound and Vibration, Vol. 235, No. 4, 2000, pp. 539-565.

[51] Gerstmayr, J. and Shabana, A. A., "Analysis of thin beams and cables using the absolute nodal co-ordinate formulation," Nonlinear Dynamics, Vol. 45, No. 1-2, 2006, pp. 109-130.

[52] Mirtich, B. and Canny, J., "Impulse-based Simulation of Rigid Bodies," Symposium on Interactive 3D Graphics, 1995, pp. 181-188.

[53] Mirtich, B. V., Impulsebased Dynamic Simulation of Rigid Body Systems, Ph.D. thesis, 1996.

[54] Chang, B. and Colgate, J. E., "Real-Time Impulse-Based Simulation of Rigid Body Systems for Haptic Display," Proceedings of the 1997 ASME International Mechanical Engineering Congress and Exhibition, 1997, pp. 8.

[55] Bender, J., "Impulse-based dynamic simulation in linear time," Computer Animation and Virtual Worlds, Vol. 18, No. 4-5, 2007, pp. 225-233.

[56] Reed, J. and Barraclough, S., "Development of Harpoon System for Capturing Space Debris," 6th European Conference on Space Debris, No. August, 2013, pp. $22-25$. 


\section{Appendix A}

\section{Supplemental Figures}

This appendix contains all tether node absolute residual boxplots of each experimental tether deployment trial that is not included in the main body of this thesis.

\section{A.1 Experimental Tether Deployment Absolute Residual Plots}




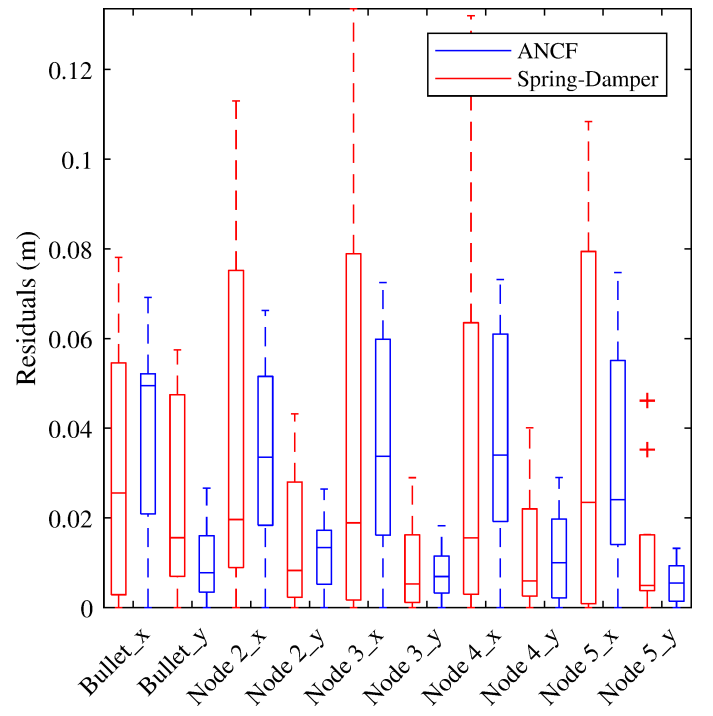

(a) Trial 1 Sub-Tether 1 Residuals

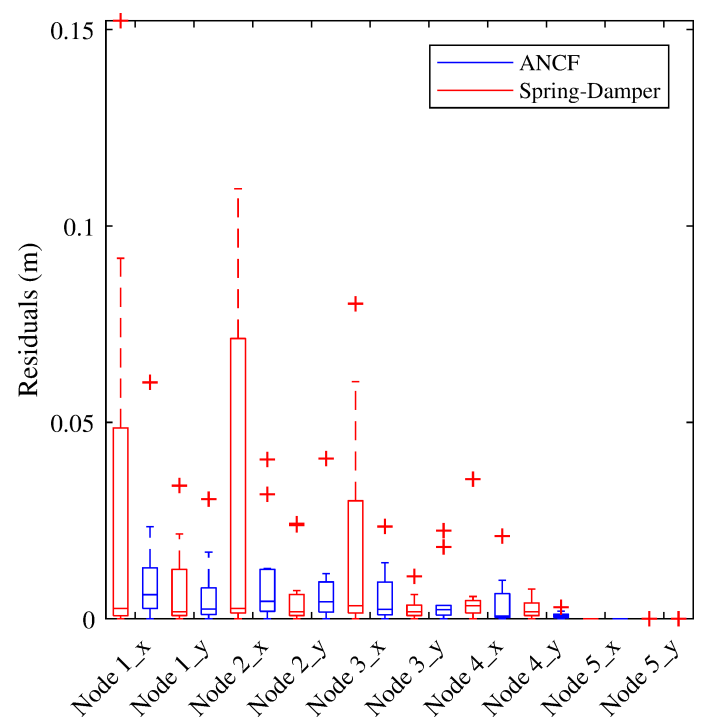

(c) Trial 1 Main Tether Residuals

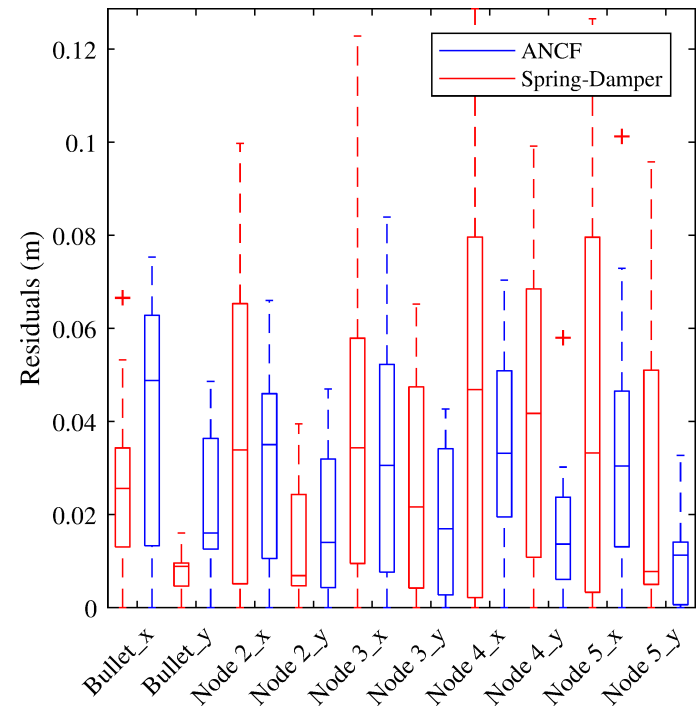

(b) Trial 1 Sub-Tether 2 Residuals

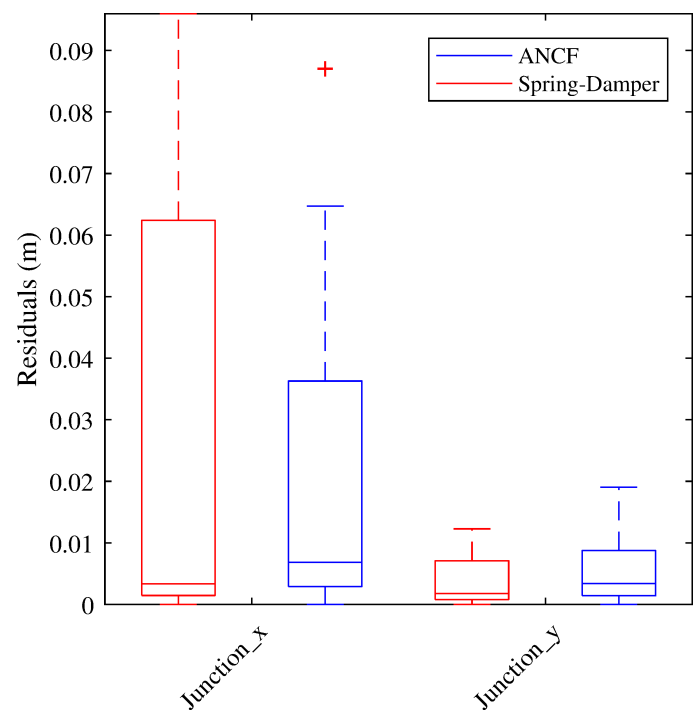

(d) Trial 1 Junction Residuals

Figure A.1: Trial 1 individual tether node absolute residuals over the duration of tether deployment. 


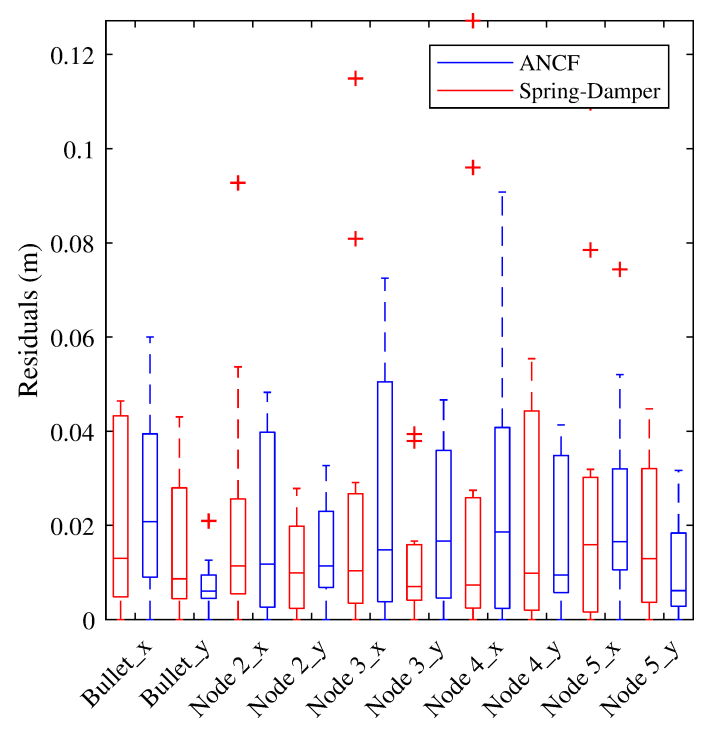

(a) Trial 2 Sub-Tether 1 Residuals

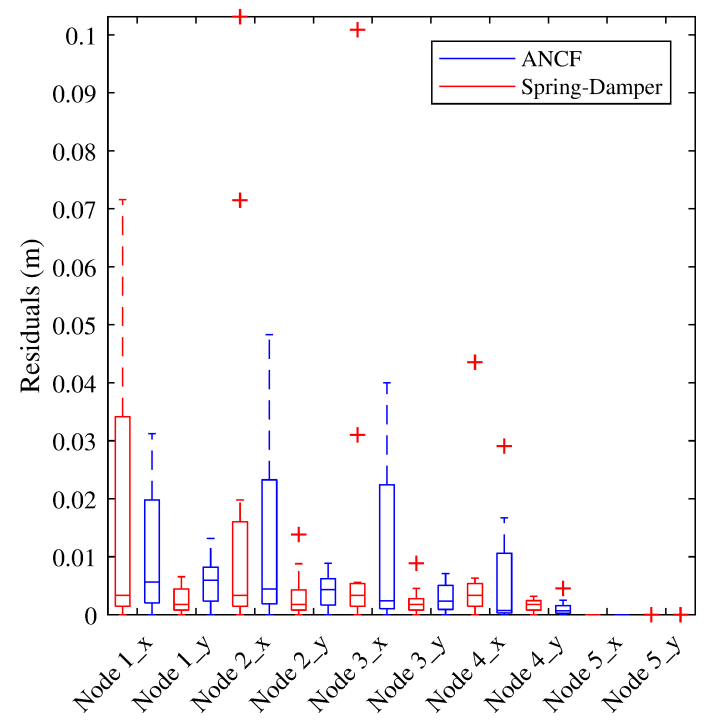

(c) Trial 2 Main Tether Residuals

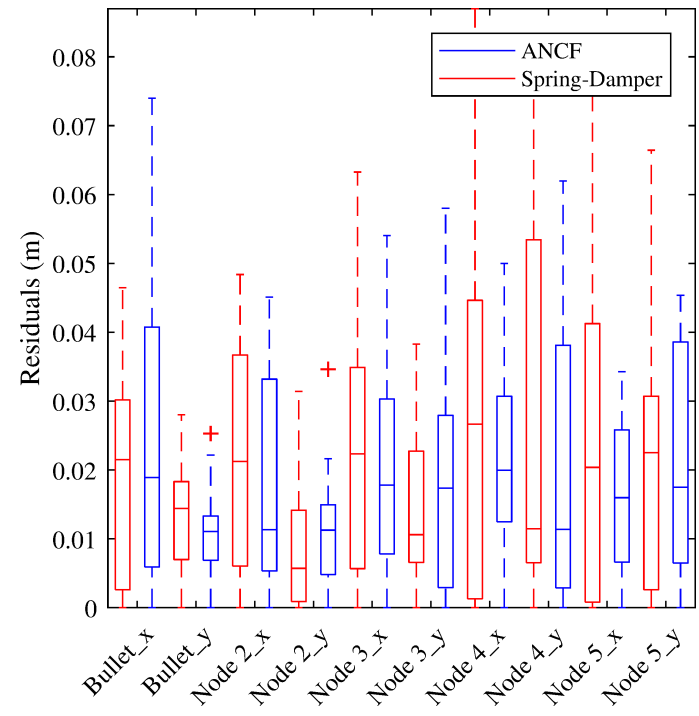

(b) Trial 2 Sub-Tether 2 Residuals

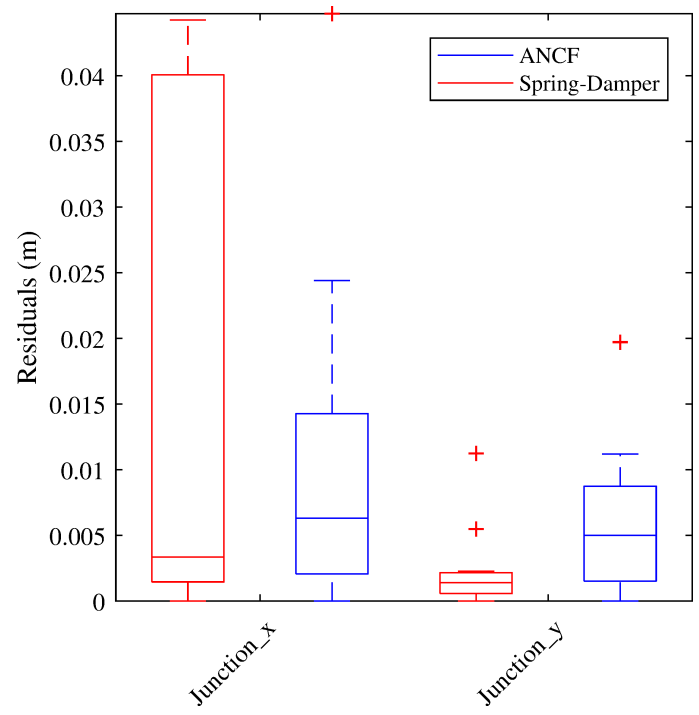

(d) Trial 2 Junction Residuals

Figure A.2: Trial 2 individual tether node absolute residuals over the duration of tether deployment. 


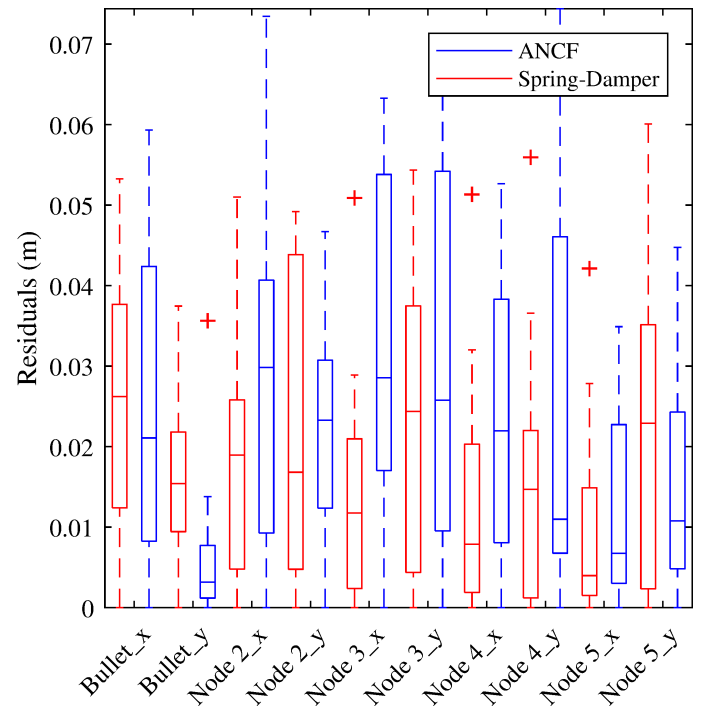

(a) Trial 4 Sub-Tether 1 Residuals

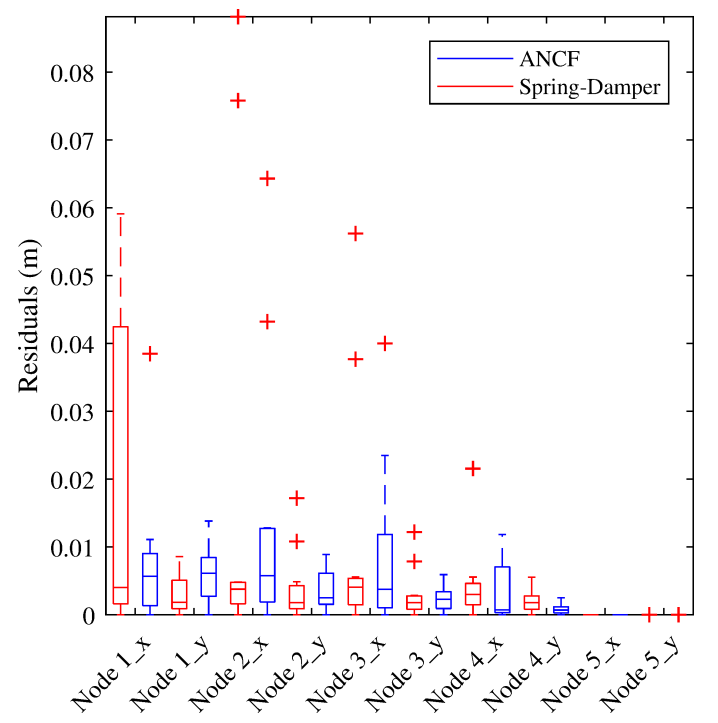

(c) Trial 4 Main Tether Residuals

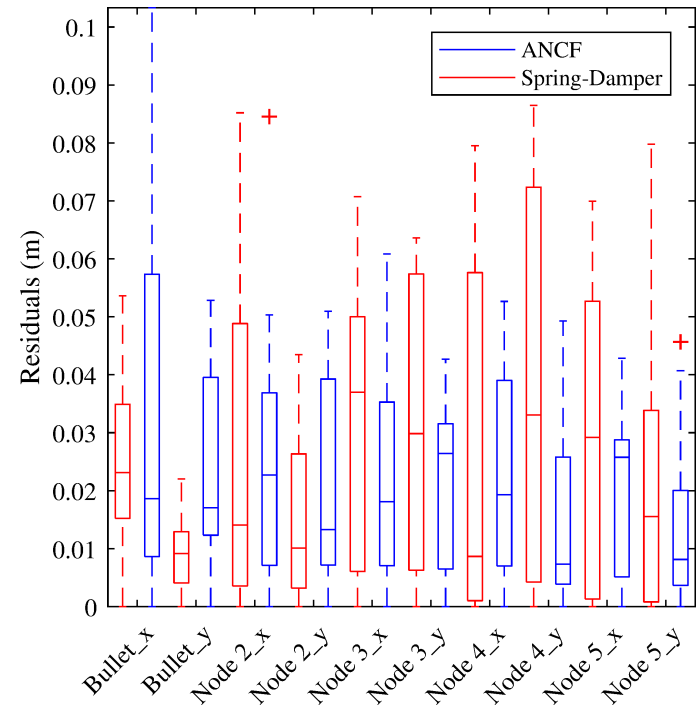

(b) Trial 4 Sub-Tether 2 Residuals

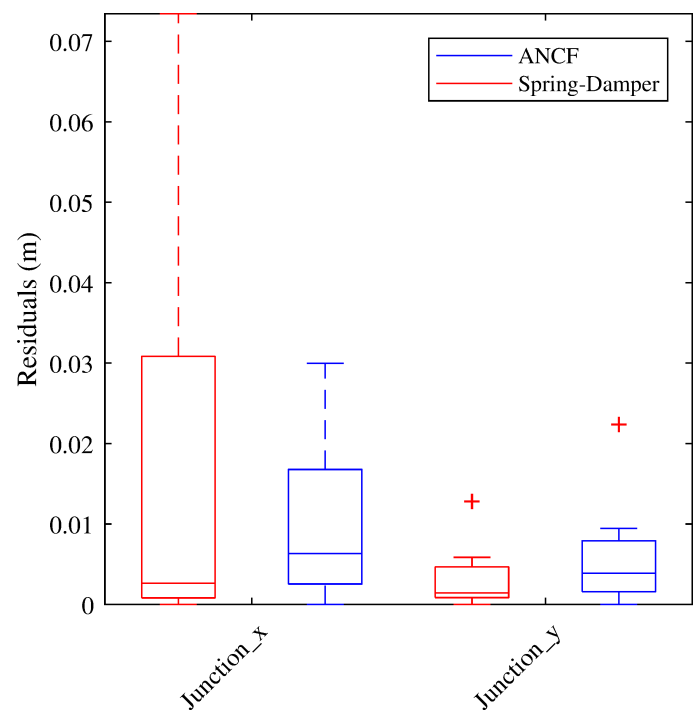

(d) Trial 4 Junction Residuals

Figure A.3: Trial 4 individual tether node absolute residuals over the duration of tether deployment. 


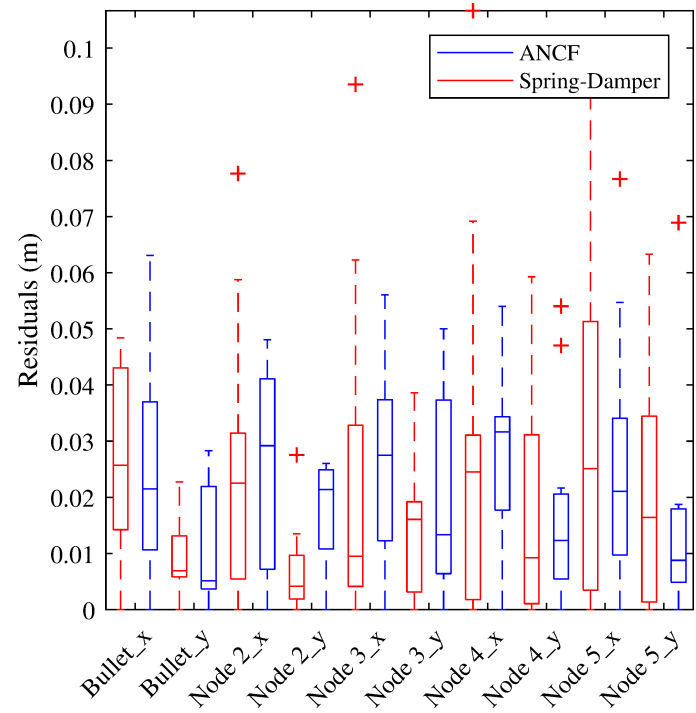

(a) Trial 5 Sub-Tether 1 Residuals

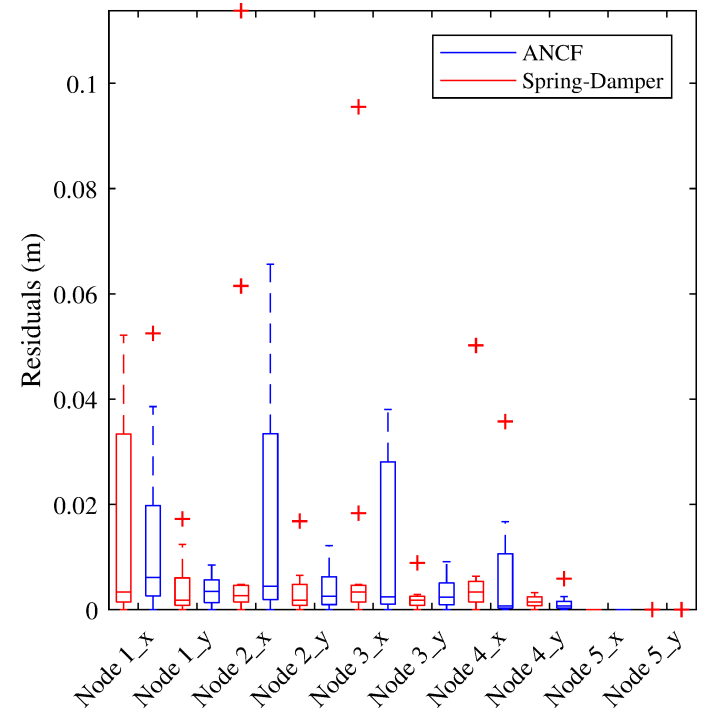

(c) Trial 5 Main Tether Residuals

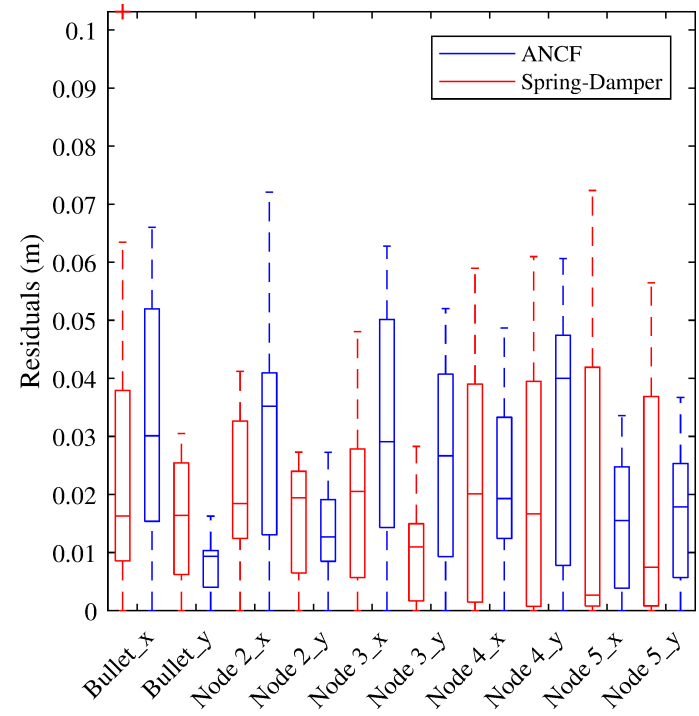

(b) Trial 5 Sub-Tether 2 Residuals

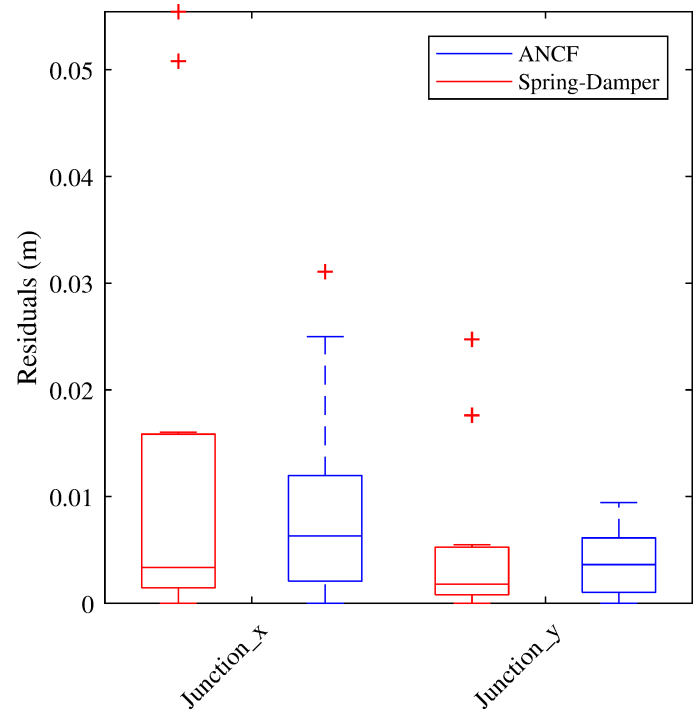

(d) Trial 5 Junction Residuals

Figure A.4: Trial 5 individual tether node absolute residuals over the duration of tether deployment. 


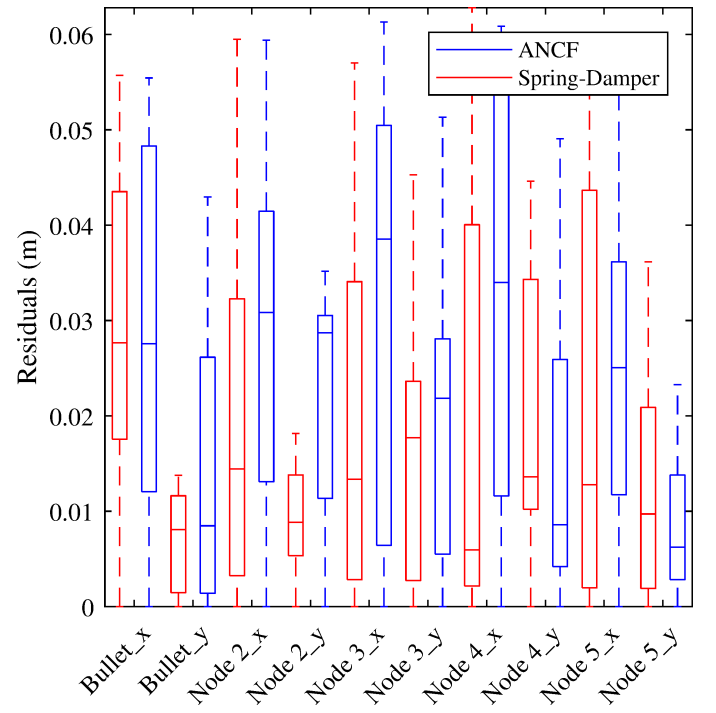

(a) Trial 6 Sub-Tether 1 Residuals

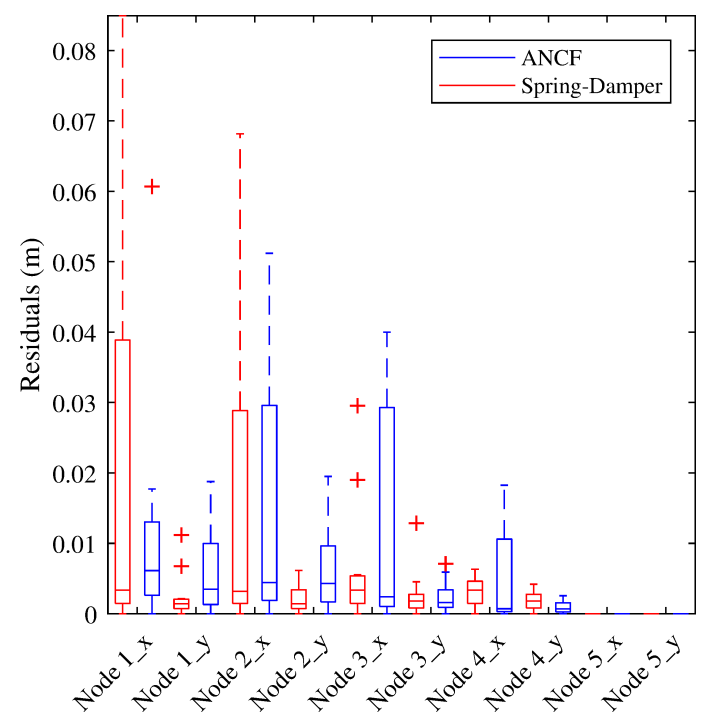

(c) Trial 6 Main Tether Residuals

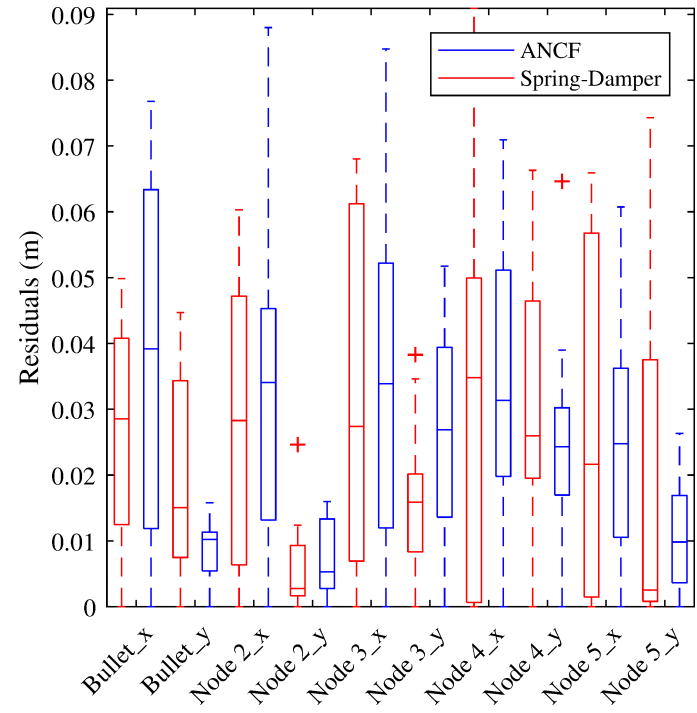

(b) Trial 6 Sub-Tether 2 Residuals

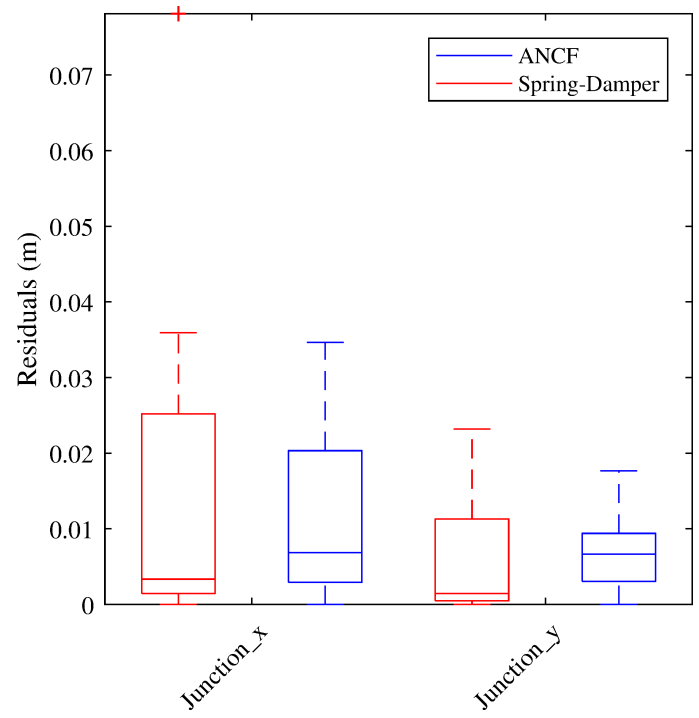

(d) Trial 6 Junction Residuals

Figure A.5: Trial 6 individual tether node absolute residuals over the duration of tether deployment. 


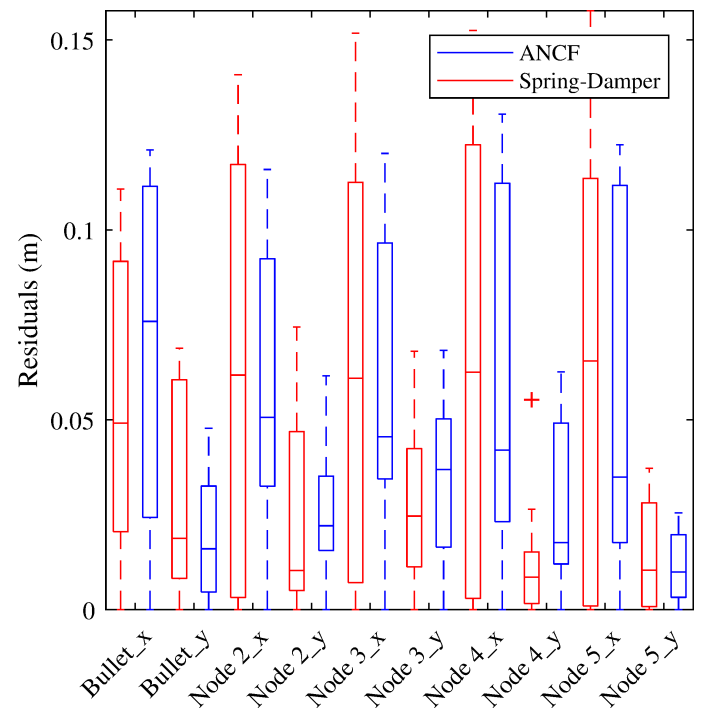

(a) Trial 7 Sub-Tether 1 Residuals

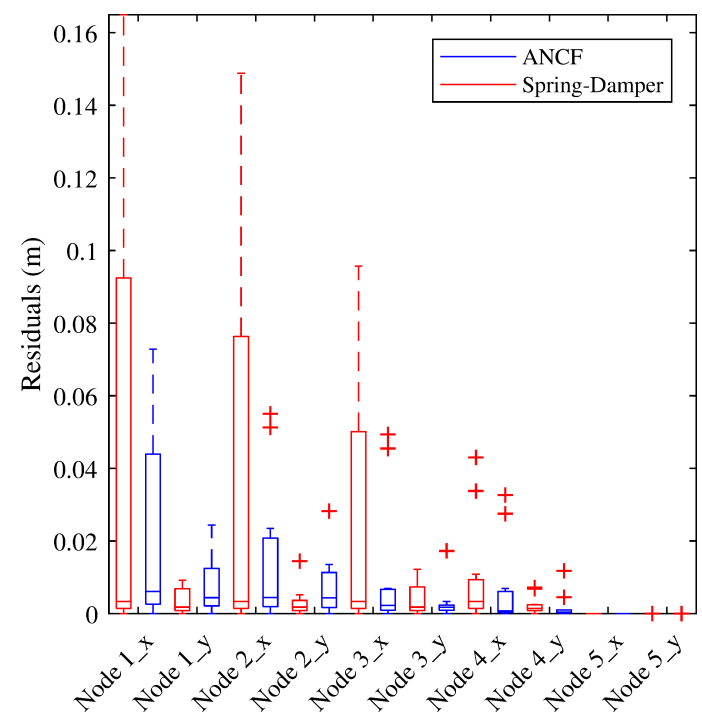

(c) Trial 7 Main Tether Residuals

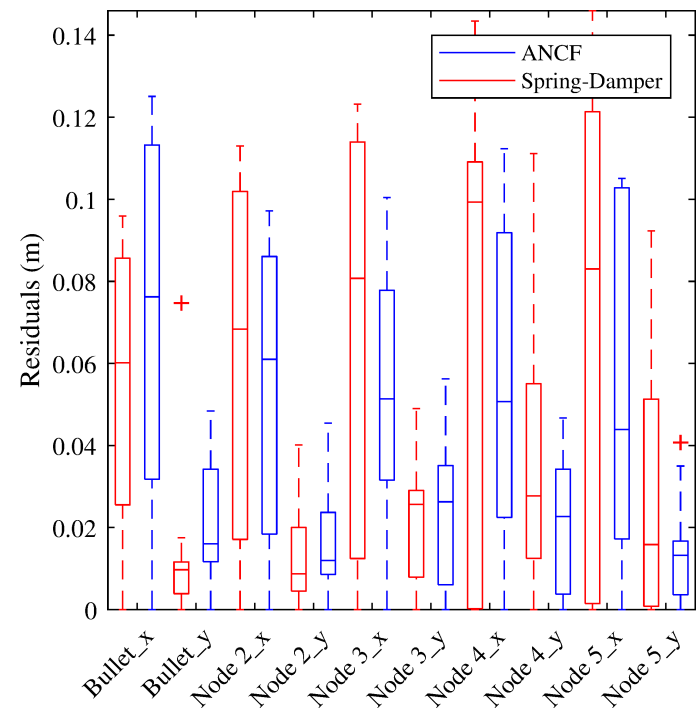

(b) Trial 7 Sub-Tether 2 Residuals

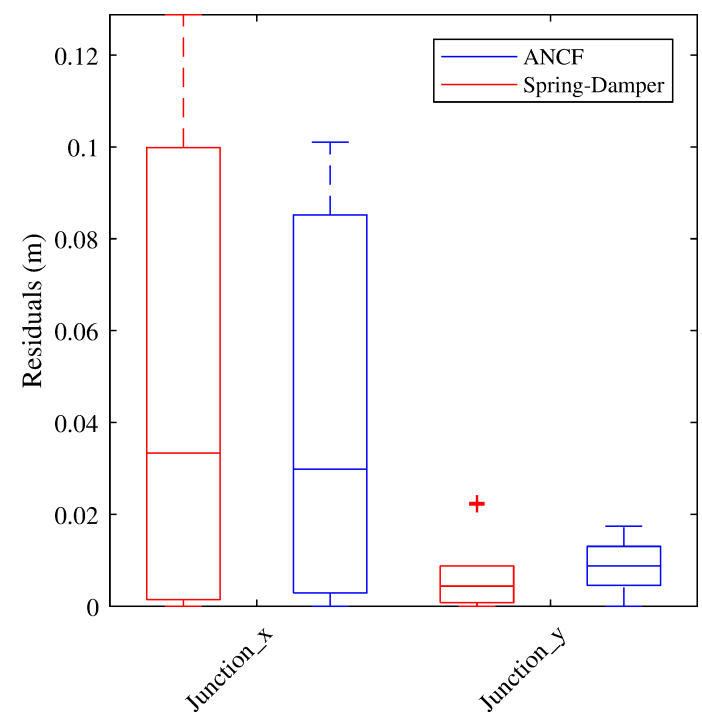

(d) Trial 7 Junction Residuals

Figure A.6: Trial 7 individual tether node absolute residuals over the duration of tether deployment. 


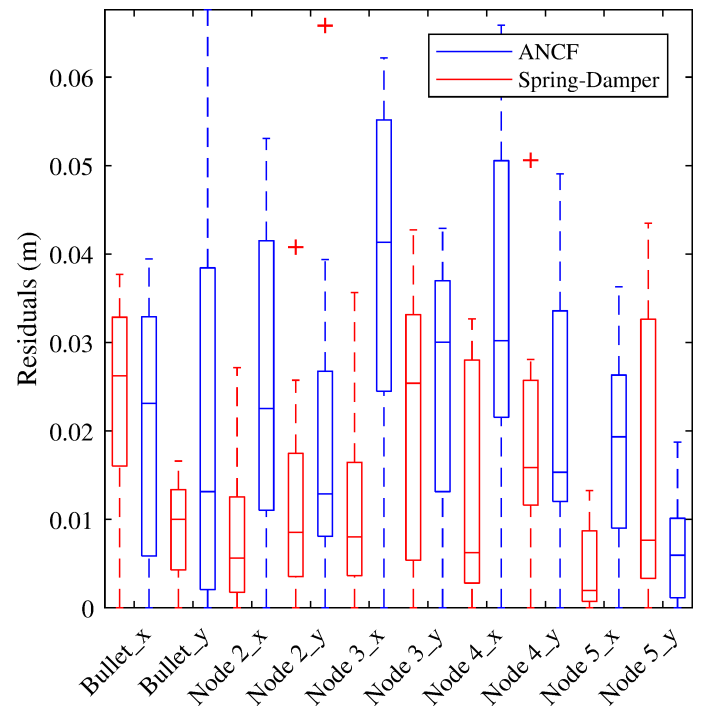

(a) Trial 8 Sub-Tether 1 Residuals

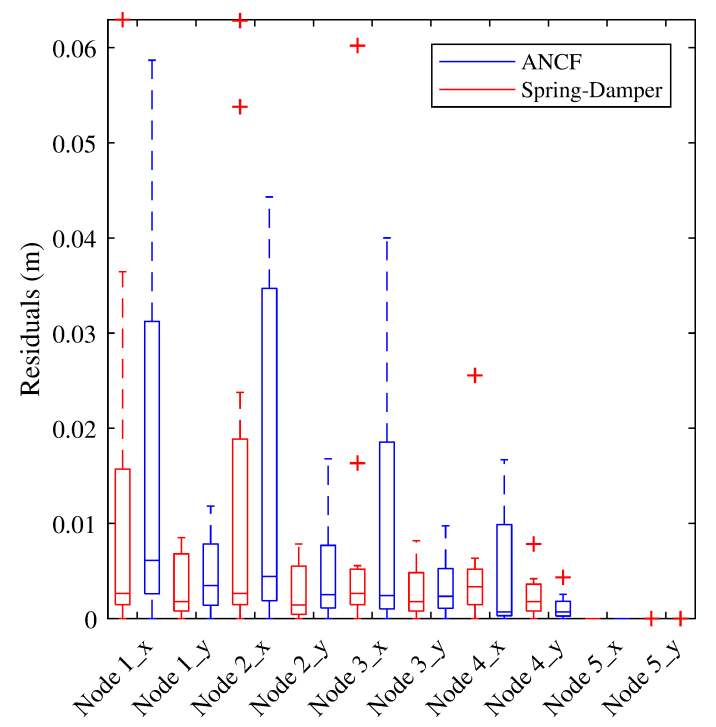

(c) Trial 8 Main Tether Residuals

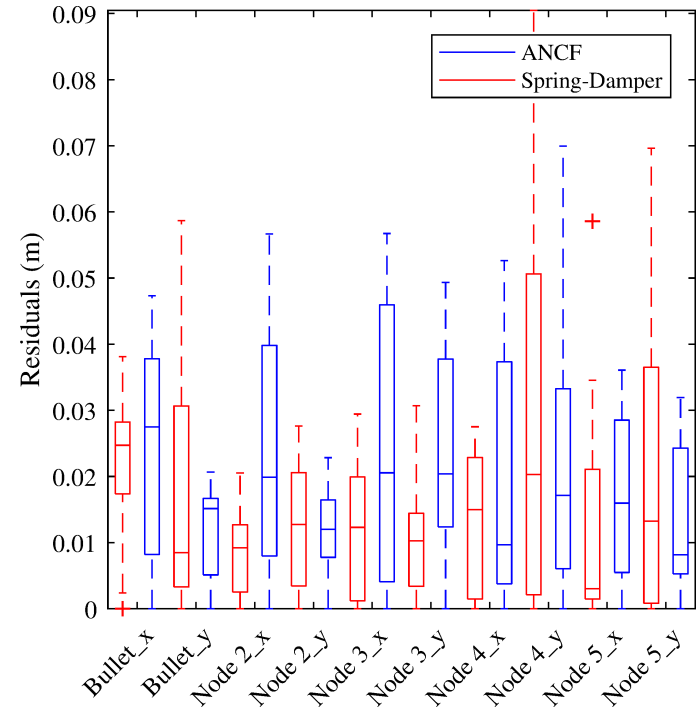

(b) Trial 8 Sub-Tether 2 Residuals

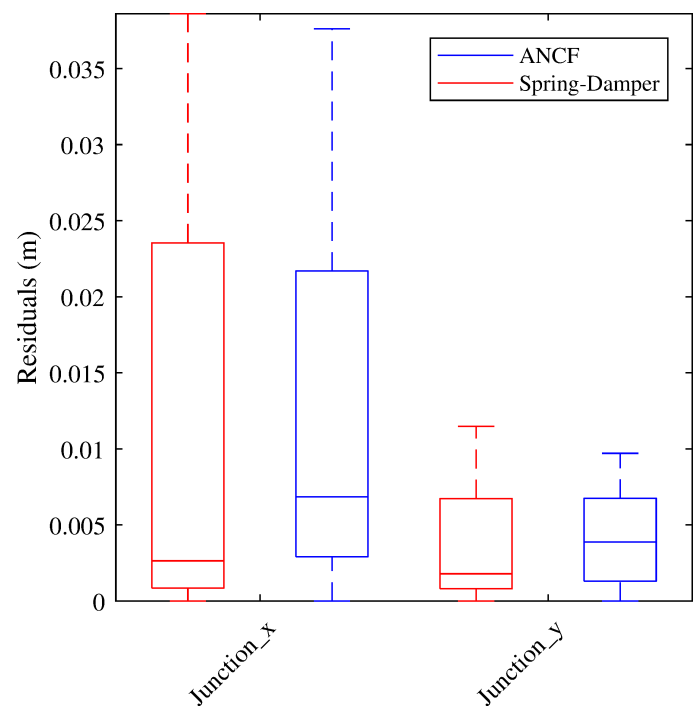

(d) Trial 8 Junction Residuals

Figure A.7: Trial 8 individual tether node absolute residuals over the duration of tether deployment. 


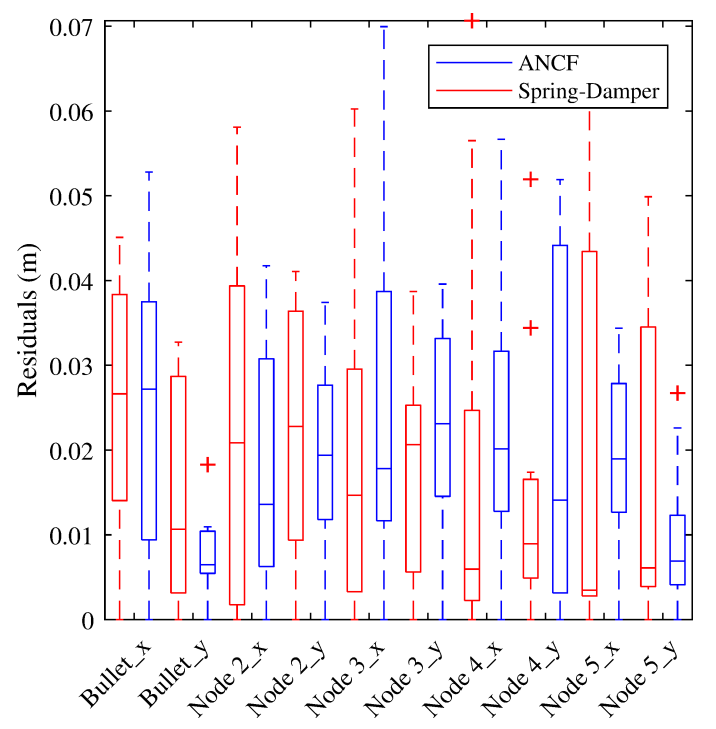

(a) Trial 9 Sub-Tether 1 Residuals

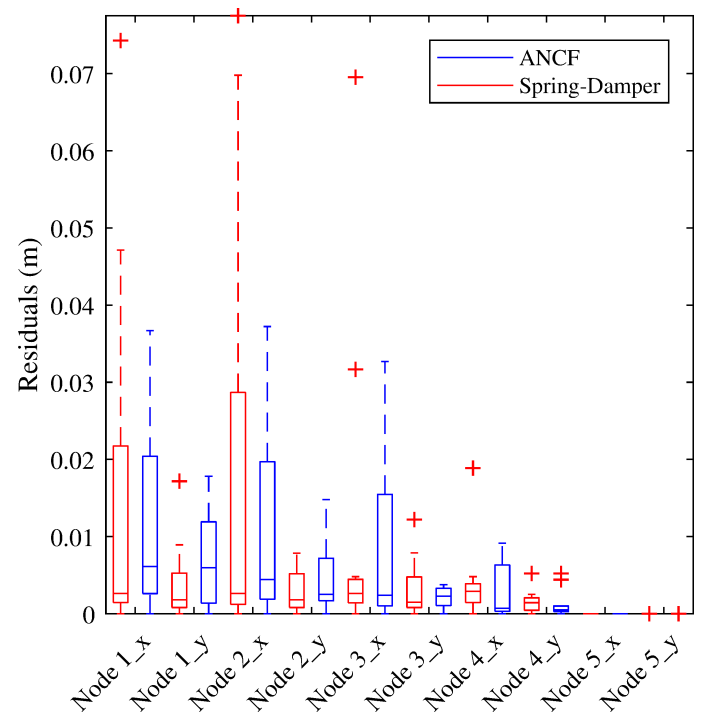

(c) Trial 9 Main Tether Residuals

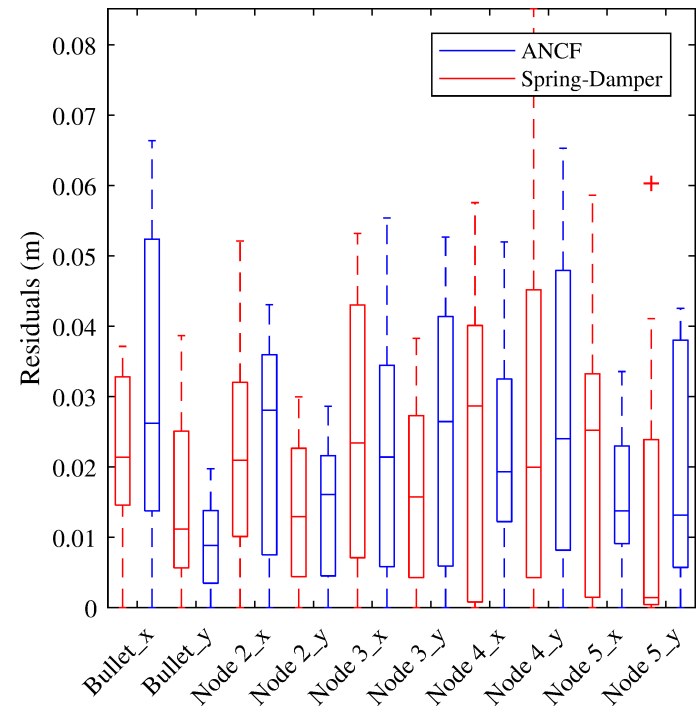

(b) Trial 9 Sub-Tether 2 Residuals

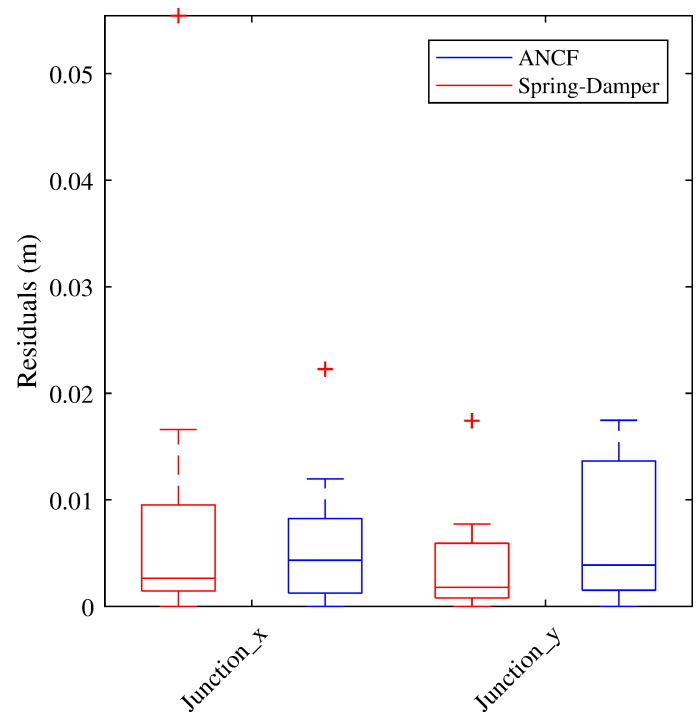

(d) Trial 9 Junction Residuals

Figure A.8: Trial 9 individual tether node absolute residuals over the duration of tether deployment. 


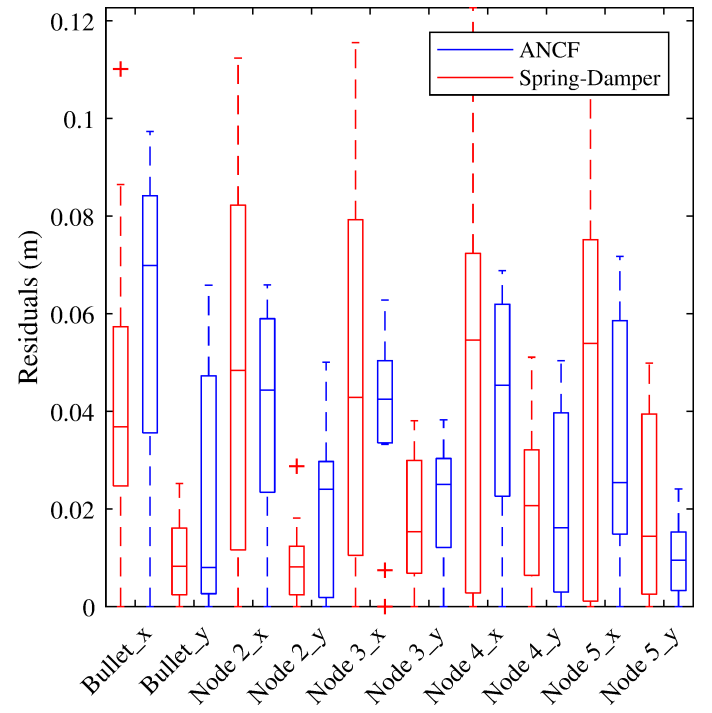

(a) Trial 10 Sub-Tether 1 Residuals

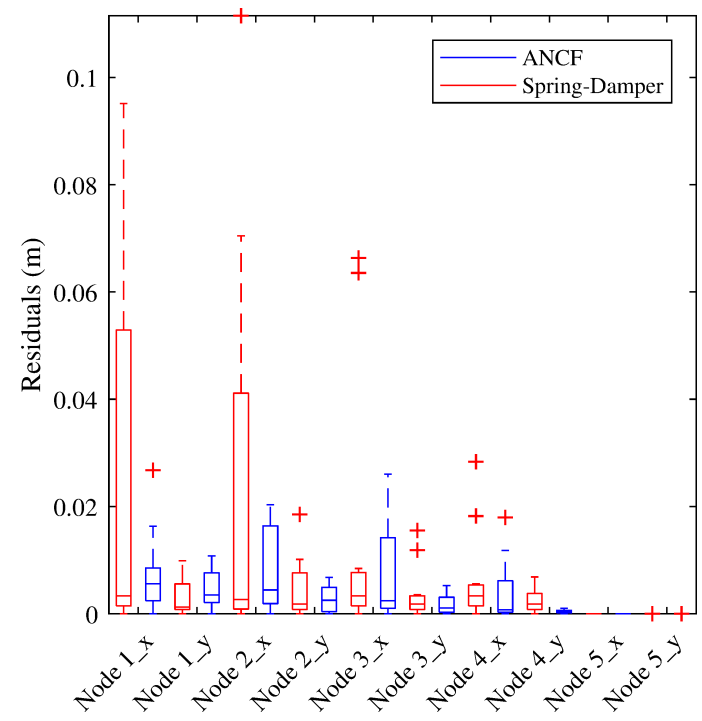

(c) Trial 10 Main Tether Residuals

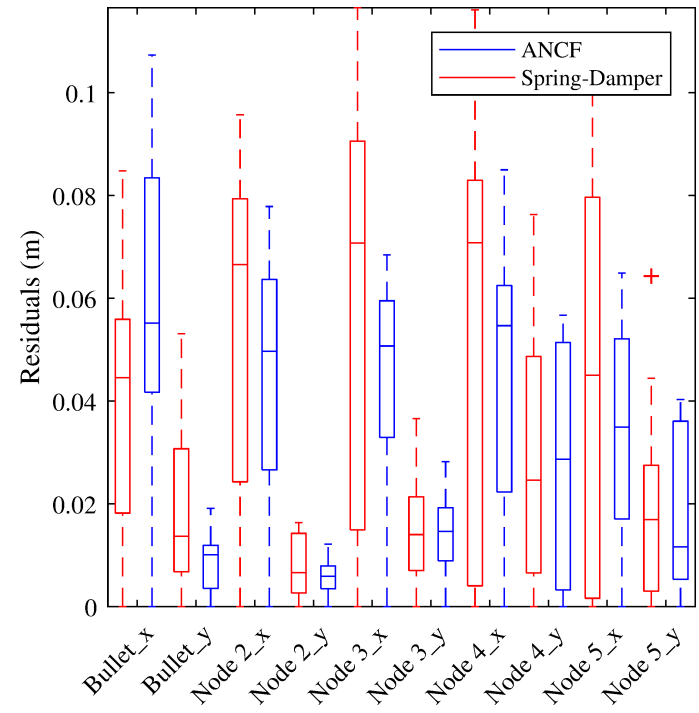

(b) Trial 10 Sub-Tether 2 Residuals

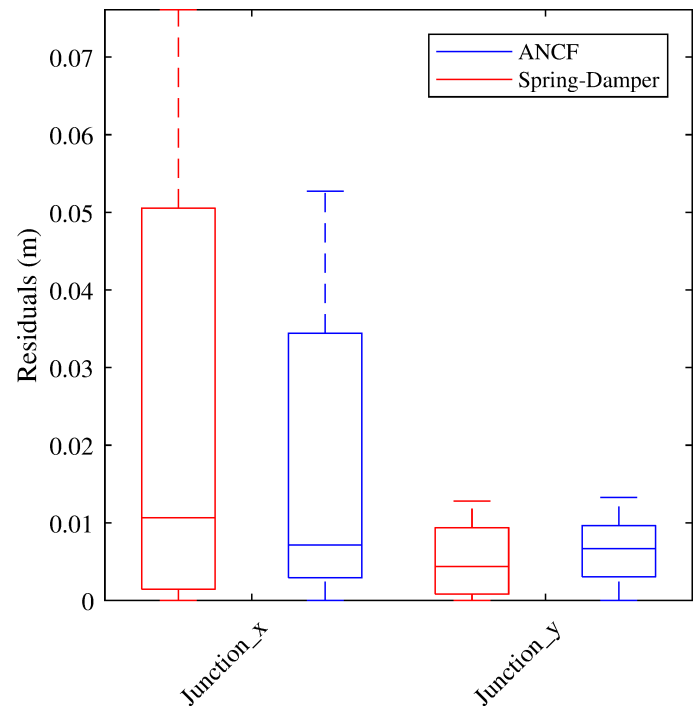

(d) Trial 10 Junction Residuals

Figure A.9: Trial 10 individual tether node absolute residuals over the duration of tether deployment. 